\title{
WestVirginiaUniversity
}

THE RESEARCH REPOSITORY @ WVU

Graduate Theses, Dissertations, and Problem Reports

2006

\section{Phytoformations of silver and gold nanoparticles}

Brooke A. Fridley

West Virginia University

Follow this and additional works at: https://researchrepository.wvu.edu/etd

\section{Recommended Citation}

Fridley, Brooke A., "Phytoformations of silver and gold nanoparticles" (2006). Graduate Theses, Dissertations, and Problem Reports. 1853.

https://researchrepository.wvu.edu/etd/1853

This Thesis is protected by copyright and/or related rights. It has been brought to you by the The Research Repository @ WVU with permission from the rights-holder(s). You are free to use this Thesis in any way that is permitted by the copyright and related rights legislation that applies to your use. For other uses you must obtain permission from the rights-holder(s) directly, unless additional rights are indicated by a Creative Commons license in the record and/ or on the work itself. This Thesis has been accepted for inclusion in WVU Graduate Theses, Dissertations, and Problem Reports collection by an authorized administrator of The Research Repository @ WVU. For more information, please contact researchrepository@mail.wvu.edu. 


\title{
Phytoformations of Silver and Gold Nanoparticles
}

\author{
Brooke A. Fridley \\ Thesis Submitted to the \\ College of Engineering \\ and Mineral Resources at \\ West Virginia University \\ in partial fulfillment of the requirements \\ for the degree of
Master of Science in Engineering in Chemical Engineering \\ Ray Y. K. Yang, Ph.D., Chair \\ Eung H. Cho, Ph.D. \\ Frank Saus, Ph.D. \\ Department of Chemical Engineering \\ Morgantown, West Virginia \\ (C) 2006
}

Keywords:

silver nanoparticles, gold nanoparticles, plant cell culture, hairy root, Pelargonium graveolens 


\title{
Abstract \\ Phytoformations of Silver and Gold Nanoparticles
}

\author{
Brooke A. Fridley
}

Stevia and red beet suspension cultures were examined for uptake of silver from $\mathrm{AgNO}_{3}$ solution. Alfalfa and mung bean sprouts were also studied for silver accumulation when grown in $\mathrm{AgNO}_{3}$ solution. AA analysis confirmed the presence of silver in both sprouts and suspension cultures after exposure to $\mathrm{AgNO}_{3}$ solution, despite the toxic effects of silver on the living matter.

Extract from Pelargonium graveolens underwent reaction with both $\mathrm{AgNO}_{3}$ and $\mathrm{KAuCl}_{4}$ to form silver or gold nanoparticles. For the reaction with $\mathrm{AgNO}_{3}$, light was required to initiate the reaction. In contrast, reactions producing gold nanoparticles did not require light to react. Oxygen appeared to have little, if any, influence on reactions involving gold or silver. The reactions were monitored for $\mathrm{pH}$ changes, and $\mathrm{pH}$ controlled reactions were also studied. Furthermore, the reaction was chemically simulated using $\mathrm{NaBH}_{4}$ as a reducing agent, yielding results similar to those observed for reactions involving geranium extract. 


\section{Acknowledgments}

I would like to express my appreciation to the many people that have assisted me in this project.

I would like to thank Dr. Ray Y. K. Yang, my research advisor, for his valuable input and guidance, and for giving me that extra push I sometimes needed. The other members of my committee, Dr. Eung Cho and Dr. Frank Saus were also there to offer helpful advice and were of great help in completion of my degree.

Furthermore, I want to thank Jim Hall for his expert ability to fix almost anything and willingness to offer a helping hand, and for providing musical entertainment on Monday evenings. I would also like to extend my gratitude to Paul Kritschgau for all his generous efforts in resolving various technical problems.

Last, but not least, I owe much to my family, who has supported me throughout my education and provided encouragement when I needed it the most. My companion, Sam Albin, has been most understanding and supportive of me, while I work late nights and long weekends.

Support for this project from the Center for Advanced Separation Technology, U.S. Department of Energy, is gratefully acknowledged. 


\section{Table of Contents}





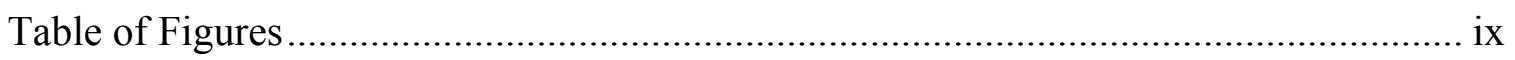

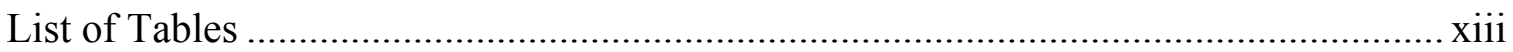

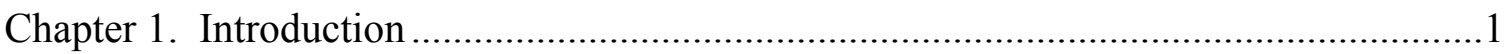

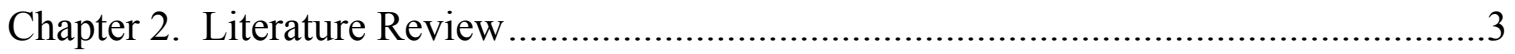

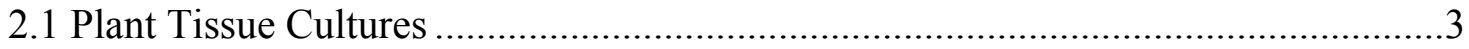

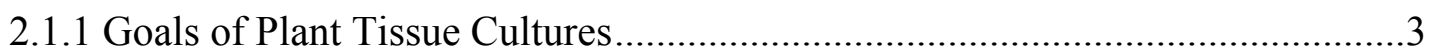

2.1.1.1 Primary and Secondary Metabolites ...............................................................

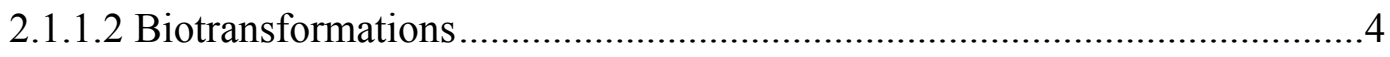

2.1.1.3 Enzyme Production ..............................................................................

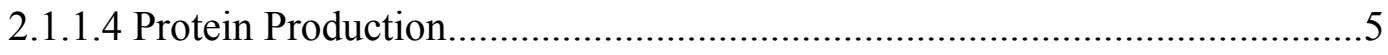

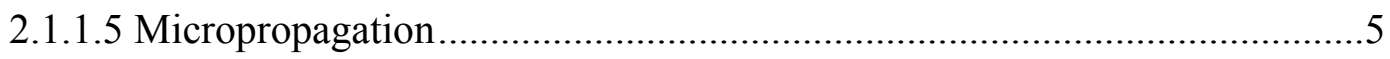

2.1.2 Types of Plant Cultures................................................................................6

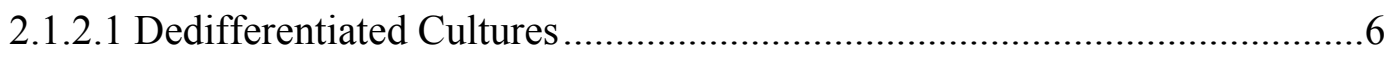

2.1.2.2 Organ Cultures ................................................................................

2.1.2.3 Hairy Root Cultures .......................................................................

2.1.3 Plants Relevant to Current Research...........................................................8

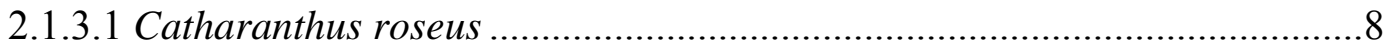

2.1.3.2 Stevia rebaudiana ………….........................................................

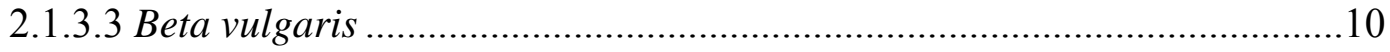


2.1.3.4 Pelargonium graveolens ..........................................................................10

2.2 Formation and Applications of Nanoparticles ....................................................11

2.2.1 Current and Future Uses of Gold and Silver Nanoparticles..............................13

2.2.2 Potential Harms of Nanotechnology .............................................................14

2.2.3 Biosynthesis of Silver and Gold Nanoparticles ..............................................15

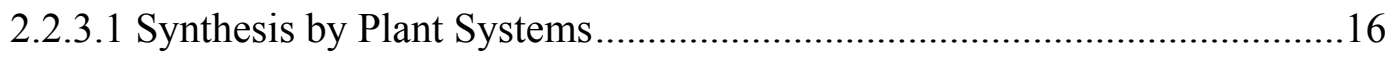

2.2.3.1.1 Intracellular Synthesis by Plant Systems ...........................................16

2.2.3.1.2 Extracellular Synthesis by Plant Systems ..........................................16



Chapter 4. Materials and Methods …………………….........................................20

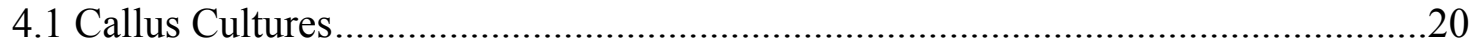

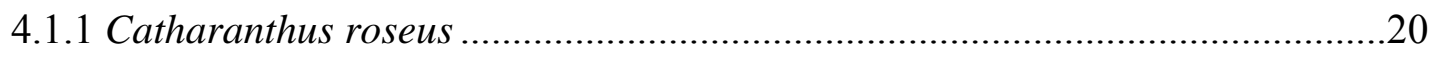

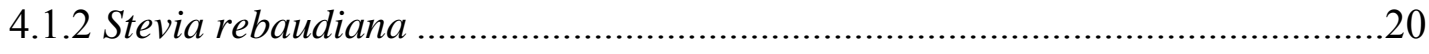



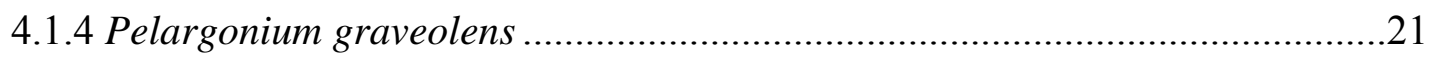

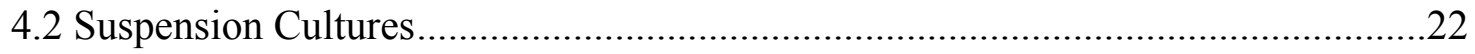

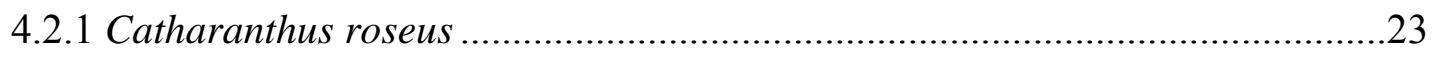

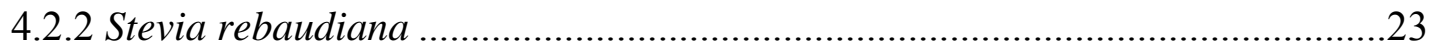

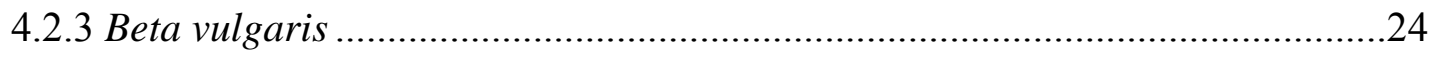



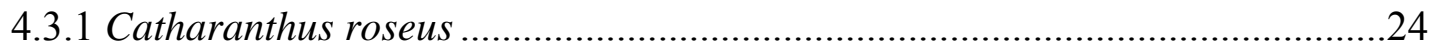

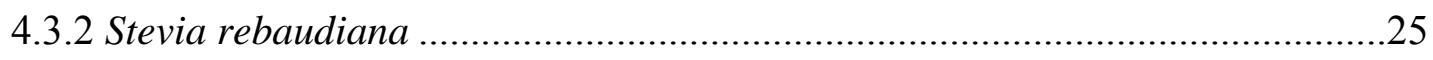




4.5 Geranium Extract

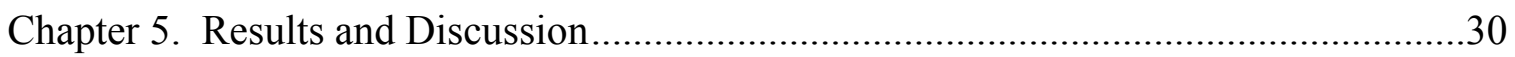

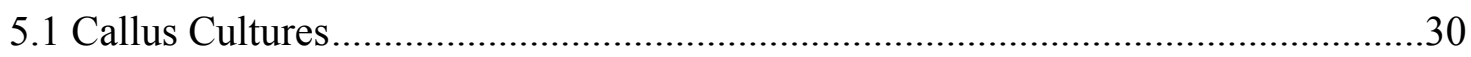

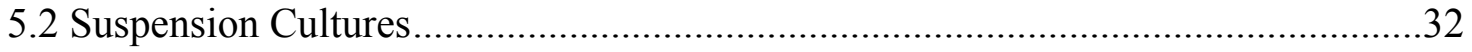

5.3 Hairy Root Cultures .................................................................................... 35

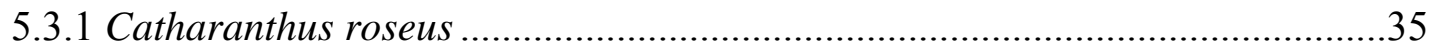

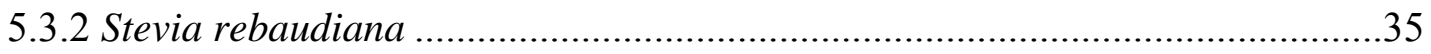

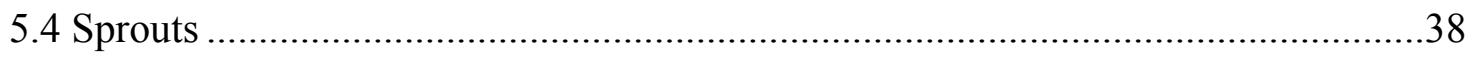

5.5 Extracellular Formation of Nanoparticles Using Pelargonium graveolens

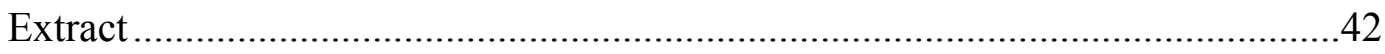

5.5.1 Formation of Silver Nanoparticles.........................................................42

5.5.2 Formation of Gold Nanoparticles ..........................................................50

5.5.3. Comparison of Biological and Chemical Methods for Nanoparticle

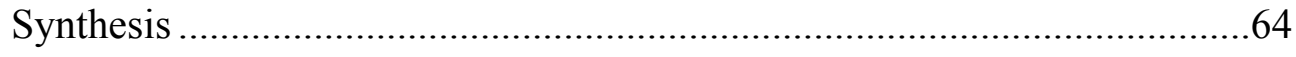

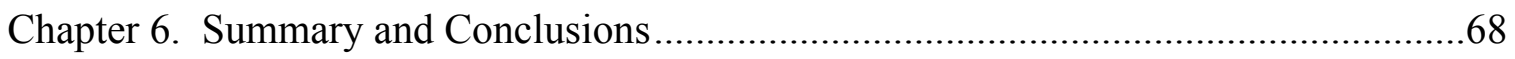

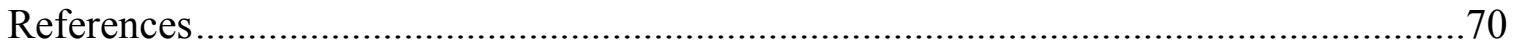



Appendix A. Methods of Sanitization ........................................................... 74

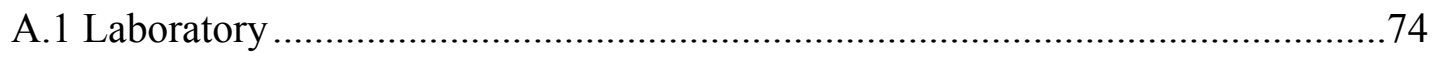



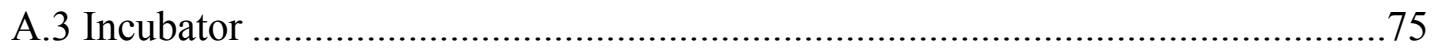

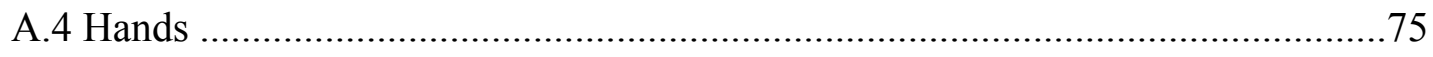

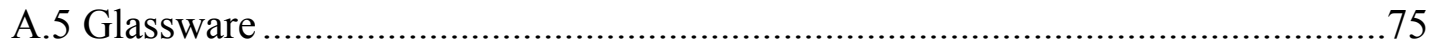


A.6 Tools

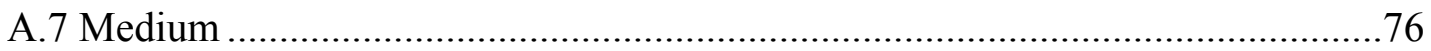

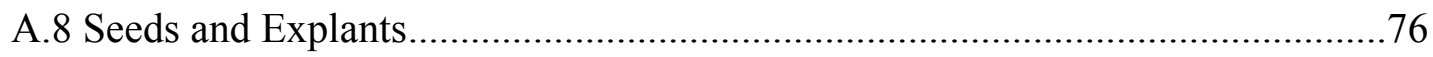

Appendix B. Procedures for Media Preparation ................................................. 78

B.1 General Procedure for Preparation of Solid Media ......................................... 78

B.2 General Procedure for Preparation of Liquid Media.......................................79

B.3 Preperation of Kinetin Solution............................................................ 79

B.4 Preparation of 2,4-Dichlorophenoxyacetic Acid (2,4-D) Solution ....................80

Appendix C. Procedures for Induction and Maintenance of Callus Cultures...............82

C.1 Induction and Maintenance of Periwinkle Callus Culture ..............................82

C.2 Induction and Maintenance of Stevia Callus Culture ........................................83

C.3 Induction and Maintenance of Geranium Callus Culture Using Leaf

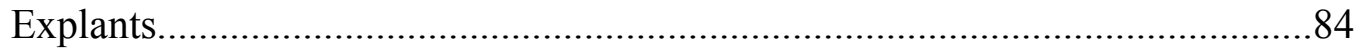

C.4 Induction and Maintenance of Geranium Callus Culture Using Stem

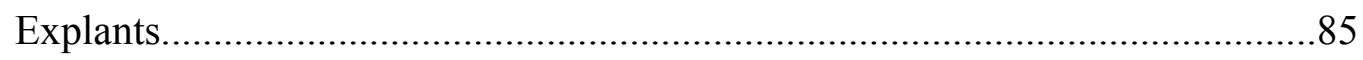

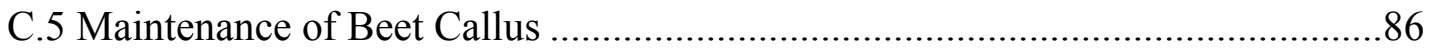

Appendix D. Procedures for Establishment and Maintenance of Suspension



D.1 Establishment and Maintenance of Stevia Suspension Culture ........................87

D.2 Establishment and Maintenance of Beet Suspension Culture...........................88

D.3 Establishment and Maintenance of Periwinkle Suspension Culture..................88

Appendix E. Procedure for Growing Suspension Cultures in $\mathrm{AgNO}_{3}$ Solution..........89

Appendix F. Filter Sterilization Using Millipore Membranes.................................91 
Appendix G. Procedures for Growing Mung Bean and Alfalfa Sprouts in Silver

Solution

G.1 Procedure for Growing Mung Bean or Alfalfa Sprouts in A-Sprouter.

G.2 Procedure for Growing Mung Bean or Alfalfa Sprouts in S-Sprouter .93

Appendix H. Procedures for Induction and Maintenance of Hairy Roots .95

H.1 Procedure for Bacteria Culture Initiation and Subculture .95

H.2 Establishment of Stevia Plantlets for Hairy Roots .95

H.3 Induction and Maintenance of Stevia Hairy Roots .96

H.4 Establishment of Periwinkle Plantlets for Hairy Roots .97

H.5 Induction and Maintenance of Periwinkle Hairy Roots........

Appendix I. Preparing Samples for Silver Analysis by Atomic Absorption .99

Appendix J. Reactions of Geranium Extract with Silver and Gold Solution 100

J.1 Geranium Reaction with $\mathrm{AgNO}_{3}$ and $\mathrm{KAuCl}_{4}$ 100

J.2 Geranium Reaction with $\mathrm{KAuCl}_{4}$ Modified to Observe Effects of Boiling

Time

J.3 Geranium Reaction with $\mathrm{KAuCl}_{4}$ Modified to Observe Effects of Boiling After

Leaves are Filtered Out 102

Addendum 103 


\section{List of Figures}

Figure 5.1. Callus cultures of (a) red beet, (b) periwinkle, (c) stevia, and (d) scented geranium.

Figure 5.2. Dependence of silver loading in Stevia cells on initial silver concentration in suspension culture media.

Figure 5.3. Dependence of gold loading in Stevia cells on initial gold concentration in suspension culture media.

Figure 5.4. Hairy root cultures of Stevia 5 weeks after first subculture (a) growing in darkness, (b) grown with $18 \mathrm{hr}$ photoperiod.

Figure 5.5. Stevia hairy root cultures grown under $18 \mathrm{hr}$ light, 8 days after subculturing from solid media to liquid media.

Figure 5.6. Dependence of silver loading in mung bean and alfalfa sprouts on initial silver concentration in sprouter solutions for type A-sprouter.

Figure 5.8. Alfalfa sprouts grown in A-sprouter for 7 days with $20 \mathrm{ppm} \mathrm{Ag}^{+}$solution...40

Figure 5.9. Reactions of $R, R R$, and LP extracts with $A_{g N O}$ pictured at (a) $t_{d}=0, t_{1}=5$

$\mathrm{hr},(\mathrm{b}) \mathrm{t}_{\mathrm{d}}=5 \mathrm{hr}, \mathrm{t}_{\mathrm{l}}=0$, (c) $\mathrm{t}_{\mathrm{d}}=0, \mathrm{t}_{\mathrm{l}}=5 \mathrm{hr}$, (d) $\mathrm{t}_{\mathrm{d}}=5 \mathrm{hr}, \mathrm{t}_{\mathrm{l}}=0$, (e) $\mathrm{t}_{\mathrm{d}}=0, \mathrm{t}_{1}=24$

$\mathrm{hr}$, (f) $t_{d}=24 \mathrm{hr}, \mathrm{t}_{1}=0,(\mathrm{~g}) \mathrm{t}_{\mathrm{d}}=0, \mathrm{t}_{\mathrm{l}}=24 \mathrm{hr},(\mathrm{h}) \mathrm{t}_{\mathrm{d}}=24 \mathrm{hr}, \mathrm{t}_{1}=0$, and at (i) $\mathrm{t}_{\mathrm{d}}$

$=0, t_{1}=26 \mathrm{hr}$ for the two jars on the left, and $\mathrm{t}_{\mathrm{d}}=26 \mathrm{hr}, \mathrm{t}_{\mathrm{l}}=0$ for the two

jars on the right.

Figure 5.10. Reaction of LP extract with $\mathrm{AgNO}_{3}, \mathrm{t}_{\mathrm{d}}=13$ days, shown at (a) $\mathrm{t}_{1}=1 \mathrm{hr}$, (b) $t_{1}=4 \mathrm{hr},(\mathrm{c}) \mathrm{t}_{1}=7 \mathrm{hr}$, and (d) $\mathrm{t}_{1}=26 \mathrm{hr}$, and (e) final products after $20 \mathrm{X}$ 
dilution, with the two jars on the left having $t_{d}=0, t_{1}=14$ days and the two jars on the right having $t_{d}=13$ days, $t_{1}=27 \mathrm{hr}$.

Figure 5.11. Reactions of $\mathrm{R}$ and $\mathrm{RR}$ extracts with $\mathrm{AgNO}_{3}$ after $20 \mathrm{X}$ dilution. For both (a) and (b), the two jars on the left have $t_{d}=0, t_{1}=8$ weeks $+24 \mathrm{hr}$, and the two jars on the right have $t_{d}=8$ weeks, $t_{1}=24 \mathrm{hr}$.

Figure 5.12. Final products from reactions of $\mathrm{R}, \mathrm{RR}$, and $\mathrm{LP}$ extracts with $\mathrm{AgNO}_{3}$ after 20X dilution. For each of (a), (b), and (c), the two jars on the left have $t_{d}=$ 0 , and the two jars on the right have $t_{d} \neq 0$.

Figure 5.13. Progression of $\mathrm{pH}$ over time upon reaction of $10 \mathrm{ml} \mathrm{R}$ extract with $100 \mathrm{ml}$ $0.001 \mathrm{M} \mathrm{AgNO}_{3}$ solution.

Figure 5.14. Progression of $\mathrm{pH}$ over time upon reaction of $10 \mathrm{ml} \mathrm{LP}$ extract with $100 \mathrm{ml}$ $0.001 \mathrm{M} \mathrm{AgNO}_{3}$ solution. .50

Figure 5.15. Reactions of RR and LP extracts with $\mathrm{KAuCl}_{4}$ showing (a) $\mathrm{t}_{d}=0, \mathrm{t}_{1}=20$ $\min (b) t_{d}=20 \min , t_{1}=0$ (c) $t_{d}=0, t_{1}=65$ min for the two jars on the left, and $t_{d}=65$ min, $t_{1}=0$ for the two jars on the right $(d)$ final RR products diluted 20X, (e) $t_{d}=0, t_{1}=5 \min (f) t_{d}=0, t_{1}=30$ min, and (g) final LP products diluted $20 \mathrm{X}$

Figure 5.16. Reactions of LP, R, and RR extracts with $\mathrm{KAuCl}_{4}$ showing (a) $\mathrm{t}=30 \mathrm{~min}$, (b) $t=40$ min, (c) final LP products diluted 20X, (d) final R products diluted 20X, and (e) final RR products diluted 20X. All jars labeled 1 and 2 reacted in lighted conditions, and jars 3 and 4 all underwent reaction in darkness. 
Figure 5.17. Reactions of $R, R R$, and $L P$ extracts with $\mathrm{KAuCl}_{4}$ showing (a) $t=10 \mathrm{~min}$, (b) $t=12 \min ,(c) t=15$ min, (d) final $R$ products diluted 20X, (e) (f) $t=5$ min, $(\mathrm{g}) \mathrm{t}=30 \mathrm{~min}$, (h) final LP products diluted 20X. All jars labeled 1 and 2 reacted in lighted conditions, and jars 3 and 4 all underwent reaction in darkness. Jars 1 and 3 were exposed to excess oxygen, while jars 2 and 4 reacted in a limited oxygen environment .55

Figure 5.18. Reactions of $\mathrm{R}$ and $\mathrm{RR}$ extracts with $\mathrm{KAuCl}_{4}$ showing (a) $t=15$ min, and (b) $\mathrm{t}=60 \mathrm{~min}$.

Figure 5.19. Reactions of $\mathrm{RR}, \mathrm{R}$, and $\mathrm{LP}$ extracts with $\mathrm{KAuCl}_{4}$ showing final products diluted 20X for (a) RR, (b) R, and (c) LP.

Figure 5.20. Absorbance spectra of samples of a solution containing gold nanoparticles (a) after reaction of $\mathrm{R}$ extract with $\mathrm{KAuCl}_{4}$, and (b) after reaction of $\mathrm{LP}$ extract with $\mathrm{KAuCl}_{4}$

Figure 5.21. Progression of $\mathrm{pH}$ over time upon reaction of $10 \mathrm{ml} \mathrm{R}$ extract with $100 \mathrm{ml}$ $0.001 \mathrm{M} \mathrm{KAuCl}_{4}$ solution.

Figure 5.22. Progression of $\mathrm{pH}$ over time upon reaction of $10 \mathrm{ml} \mathrm{LP}$ extract with $100 \mathrm{ml}$ $0.001 \mathrm{M} \mathrm{KAuCl}_{4}$ solution. .60

Figure 5.23. Products from reactions of $10 \mathrm{ml} \mathrm{LP}$ extract with $100 \mathrm{ml} 0.001 \mathrm{M} \mathrm{KAuCl}_{4}$ solution with initial reaction $\mathrm{pH}$ adjusted (from left to right) to $3.5,4.0,4.5$, and 6.0. Samples shown were diluted 20X following reaction .62

Figure 5.24. Peak wavelength from visible light spectra from reactions of geranium extract with $1 \times 10^{-4} \mathrm{M} \mathrm{AgNO}_{3}$ vs. extract boiling time. 
Figure 5.25. Peak absorbance from visible light spectra from reactions of geranium extract with $1 \times 10^{-4} \mathrm{M} \mathrm{AgNO}_{3}$ vs. extract boiling time.

Figure 5.26. (Top) Solutions of $0.001 \mathrm{M} \mathrm{AgNO}_{3}$ and $0.001 \mathrm{M} \mathrm{KAuCl}_{4}$ shown (a) before and (b) after addition of a small amount of $\mathrm{NaBH}_{4}$. (Bottom) Solutions produced upon reaction of (c) $\mathrm{AgNO}_{3}$ and (d) $\mathrm{KAuCl}_{4}$ using either $\mathrm{NaBH}_{4}$, LP extract, or R extract as the reducing agent. ...........................................66

Figure A.1. Absorbance spectra of samples of a solution containing gold nanoparticles after reaction of $\mathrm{RR}$ extract with $\mathrm{KAuCl}_{4}$, (a) shown without dilution, and (b) diluted $2 \mathrm{X}$ relative to the sample represented in (a) 104 


\section{List of Tables}

Table 5.1. Peak wavelengths from spectra of silver nanoparticles produced by the reaction of extract from various cultivars of $P$. graveolens with $0.001 \mathrm{M}$

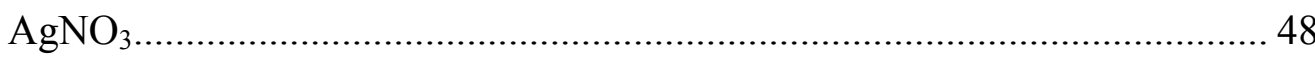

Table 5.2. Peak wavelengths from spectra of gold nanoparticles produced by the reaction of extract from various cultivars of $P$. graveolens with $0.001 \mathrm{M} \mathrm{KAuCl}_{4} \ldots . .56$

Table B.1. Components required for preparation of various media 81 


\section{Chapter 1. Introduction}

Plants have been essential to humans since the beginning of time. While plants still serve to fulfill some of mankind's basic needs such as food, oxygen, and shelter, new roles of plants are continuously being discovered. Plants contain multitudes of compounds with applications that range from use as fragrances or dyes to treatment of illness and diseases. Plants also have the potential to carry out complex chemical reactions. Recently, there has been an increase in research aiming to understand and take advantage of these extraordinary capabilities.

This body of work encompasses many topics involving biochemical processes that employ plants and plant systems. One such bioprocess that has been observed is the ability for some types of plant cell and tissue culture systems to uptake metal ions from solution. In this research, callus cultures, cell suspension cultures, and hairy root cultures of several different plants will be initiated, established, maintained and used. Two types of plant sprouts, as well, will be grown from seed and exposed to silver nitrate solution. Another bioreaction involved in this thesis is the biosynthesis of silver and gold nanoparticles by plant extracts. As more and more applications for nanoparticles are being discovered, there is a growing interest in developing systems for their production that are efficient, environmentally safe, and easily controlled. Plants have been proposed as a possible synthesis pathway that could potentially meet these requirements.

Existence of silver nanoparticles in alfalfa sprouts was first reported in 2003 (Gardea-Torresdey 2003). In the same year, the formation of silver nanoparticles by extract of geranium leaves was published by Shankar et al. (2003b). This group has 
carried out various experiments on production of silver and gold nanoparticles by several different species of plants. One such plant that has shown much potential for rapid nanoparticle production is Pelargonium graveolens, commonly known as scented geranium. The extract of this plant has been shown to reduce silver and gold ions in solution to form nanoparticles with an assortment of shapes and sizes. While the mechanism for the reaction that occurs is not well understood, several influencing factors have been identified. Yet, many features involved in this process are still unknown, and there is much room for advancement of the techniques used.

Much of the research that will be completed for fulfillment of this thesis is intended to reveal more information about this plant-based method of silver and gold nanoparticle synthesis. Hopefully, the insight gathered from this research will contribute to future efforts in manufacturing metal nanoparticles using environmentally friendly methods. 


\section{Chapter 2. Literature Review}

\subsection{Plant Tissue Cultures}

Exploration in plant tissue cultures began over 100 years ago when Haberlandt tried the first documented in vitro plant cell culture (Höxtermann 1997). Since the 1902 publication of his experiment, major discoveries and improvements have been made that prove this area of research useful and relevant in a wide assortment of applications. Plant tissue cultures were developed initially for study of plant metabolism, physiology, and growth (Scragg 1994). Recent applications have expanded to include harvesting of natural products, biotranformations, enzyme and protein production, and micropropagation (Scragg 1994). Several fields of study, including horticulture, agriculture, botany, pharmaceutical studies and medicine, biochemistry, and chemical engineering, have much interest in plant tissue cultures for research and commercial use.

\subsubsection{Goals of Plant Tissue Cultures}

\subsubsection{Primary and Secondary Metabolites}

It has long been known that plants produce materials that can be very valuable resources. Primary metabolites (Bayraktar 1996) of plants are compounds that occur naturally in plants, and are essential in carrying out basic processes for survival of the plant cells. They are generally high-volume substances that are inexpensive and rather easy to harvest, such as vegetable oils, carbohydrates, and fatty acids. These compounds 
are of much significance in the food industry, and they frequently find uses as raw materials in manufacture of various other products.

Secondary metabolites, also naturally produced by plants, usually are present only during certain developmental stages, or are made in response to environmental stresses (Robins 1994), and are specific to each taxonomic group. These substances, which include perfumes, dyes, flavorings, and a vast array of pharmaceuticals, are typically only found in whole plants in very small amounts (Bayraktar 1996). Therefore, efforts have moved toward the use of plant cell and tissue cultures grown in specific conditions that can be customized to enhance formation of the desired products.

\subsubsection{Biotransformations}

Biotransformations refer to the countless chemical reactions that can occur with the assistance of plants. Plants contain an abundance of unique enzymes and chemicals that can react to transform one compound to another. One such instance that has currently attracted attention is the ability of certain plants to cause metal ions to undergo reduction reactions, resulting in the formation of metal nanoparticles. This concept is analyzed in more detail in Section 2.2.3.1.

\subsubsection{Enzyme Production}

Extraction of enzymes from plants is another intention of plant cell and tissue cultures (Hood and Woodard 2002). Enzymes have found applications in food processing and wood products industries. They are also used in cleaning agents, as processing reagents, and for biomass conversion. 


\subsubsection{Protein Production}

Protein production by plants has found an important position in the pharmaceutical industry. Many proteins found naturally in plants have biologically active properties. In addition, with the help of genetic engineering, plants also possess the capability to assemble human and animal proteins. Recombinant protein production in plants has many advantages over other systems like production by mammalian cells or by microorganisms (Daniell et al. 2001). Production by mammalian cells is expensive and large scale processing is not realistic. Although using animal cell cultures gives the resulting proteins properties identical to those found in natural proteins, the possibility of contamination by pathogens presents a health risk to humans. Microorganisms, on the other hand, have potential for large-scale manufacture, but products made by bacteria and fungi often lack the correct conformation needed to react properly in humans. For example, human proteins constructed by E. coli lack appropriate folding and disulfide bridges. Protein production by plants offers a more economical approach than fermentation or bioreactor systems. Plant systems can easily be scaled up, and molecular structures with suitable folding have been successfully created. Moreover, a plantcentered design has the benefit of increased safety since contamination by human pathogens in plant cells is unlikely.

\subsubsection{Micropropagation}

Another major motivation of plant tissue cultures is micropropagation of plants, which can serve a number of purposes. In vitro plant regeneration has proved to be 
economically important in situations where conventional propagation has been ineffective (Rao 1994). Tissue culture-based somaclonal variation experiments have been used as a means of finding new genetic combinations that give rise to beneficial traits. Horticulturists use tissue cultures to regenerate plants that have special ornamental characteristics, or to grow pathogen-free plants. Agriculture employs culture techniques seeking to create crops with higher yields, resistance to disease or herbicides, and tolerance to environmental and chemical stresses. For example, in India, geranium oil is a valued product, but production is limited due to lack of variability and a restricted region of growth (Saxena et al. 2000). These geraniums are susceptible to diseases and pests like white ants, and are hindered by accumulation of stagnant water during the rainy season (Gupta et al. 2002). These environmental sensitivities prevent economic viability of the crop in the plains regions of India. Development of improved plants through somaclonal variation has been presented as a possible tactic for overcoming these limitations.

\subsubsection{Types of Plant Cultures}

\subsubsection{Dedifferentiated Cultures}

Numerous forms of plant tissue cultures are commonly used in biotechnological research. Among the earliest contributions to the science of plant cell culture was the exploration of dedifferentiated cultures, including callus and cell suspension cultures. These fast growing plant cultures have been extensively researched, but are still utilized in many ventures of modern plant cell culture technology. This form of plant culture is 
often used for plant regeneration, embryogenesis, selection of traits, or development of large-scale cultures (Franklin and Dixon 1994).

\subsubsection{Organ Cultures}

Cultures that have differentiated into roots, shoots, or embryos have been able to achieve high accumulation of secondary metabolites (Robins 1994). Organ cultures display genetic and biochemical stability, whether they are genetically transformed (have been subjected to introduction, integration, and expression of foreign RNA or DNA) or untransformed. Also, organ cultures normally possess full biosynthetic abilities, which make them good candidates for harvesting secondary products.

\subsubsection{Hairy Root Cultures}

Hairy root cultures have been a focus of plant tissue research for the past two decades (Doran 2002). They are formed by genetic alteration of plant cells through use of the bacteria Agrobacterium rhizogenes. There exist hundreds of plant species that have been effectively transformed to produce these hairy root-like structures. Hairy roots have many properties that make them favorable for scientific studies. Because they demonstrate genotype and phenotype stability, they can be used to develop cultures that will remain consistent over long periods of time. Additionally, hairy roots display high growth rates on media that do not contain growth hormones, which is an advantage over untransformed roots that are not capable of surviving without exogenous growth regulators. Often, a doubling time of only 1-2 days is observed in hairy roots, which is much faster than seen in untransformed roots, and is comparable to that of suspension 
cell cultures (Doran 2002). Additionally, hairy roots are able to produce much higher levels of secondary products than seen in undifferentiated cultures.

\subsubsection{Plants Relevant to Current Research}

This study of plant cultures focused on establishment, maintenance, and analysis of callus culture, cell suspension culture, and hairy root cultures only.

\subsubsection{Catharanthus roseus}

Callus cultures were induced from several species of plants. Catharanthus roseus, common name Madagascar periwinkle, is one of the most broadly studied plants for plant tissue culture. Two of the constituents of periwinkle, vinblastine and vincristine, play a significant role in the treatment of cancer. Other components of this plant have been useful in treatment of hypertension (Scragg 1994). Bayraktar (1996) provides detailed information regarding methods for establishment of callus and suspension culture of $C$. roseus.

\subsubsection{Stevia rebaudiana}

Stevia rebaudiana, another plant that was cultured in this research, produced callus by procedures according to Swanson et al. (1992). Suspension was developed by transferring callus to a medium that is similar to that used for callus maintenance, except agar is omitted, so the medium is in liquid form. Stevia has only recently been used in plant tissue cultures, with the rise of interest in safe low-calorie alternatives to sugar. Stevia's secondary metabolites, stevioside and rebaudioside, have been found to have a 
pleasant sweet taste, and in contrast to many synthetic sugar substitutes, no harmful side effects have been observed (Megeji et al. 2005). The intensity of sweetness of stevioside was determined to be 300 times greater than that of sucrose with $0.4 \%$ concentration (Swanson et al. 1992). This product, already commercially available, is recommended for diabetics (Megeji et al. 2005), and some studies have indicated that Stevia has a hypoglycemic effect (Savita et al. 2004). In the past, Stevia propagation by seeds has been limited because the plants generated show great variability in sweetening levels and other plant qualities (Sivarum and Mukundan 2003). Vegetative plant reproduction can reduce variability, but is a slow process. Using tissue cultures is the best strategy for overcoming these difficulties in rapid mass cultivation of this plant. Research has shown some potential for stevioside and rebaudioside production from callus that is grown on media supplemented with growth regulators (Sivarum and Mukundan 2003). However, conflicting results have been presented by other groups of researchers suggesting that differentiation must occur as a precursor to production of these compounds (Yamazaki and Flores 1991, Swanson et al. 1992).

Researchers have successfully accomplished hairy root transformation of Stevia via Agrobacterium rhizogenes in the past. It has been primarily used as a method for studying synthesis of two steviol glucosides, stevioside and rebaudioside. Hairy root cultures were developed in the present study based on processes detailed by Yamazaki and Flores (1991). 


\subsubsection{Beta vulgaris}

Beta vulgaris is another species that has been studied extensively through plant culture experimentation. It has been valued commercially for its sugar content as well as its pigments. Betacyanins, red pigments, and betaxanthins, yellow pigments, give the characteristic deep red-purple color of beets (Bayraktar 1996). These pigments belong to a group of compounds called betalains, which are used as food colorings. Sugar extracted from beets accounts for approximately $40 \%$ of the world's sugar manufacture (University of Iowa Healthcare 2006). Plant culture techniques have been employed to search for improvements in harvesting of these compounds. Relevant culture techniques have been described in a multitude of scientific papers. In this study, callus and suspension methods were adopted from Bayraktar (1996) and Beach (1998).

\subsubsection{Pelargonium graveolens}

Scented geraniums (Pelargonium sp.) have long been valued worldwide as ornamental plants, and propagation of this crop has become a mulit-million dollar industry (KrishnaRaj et al. 1997). The aromatic oils extracted from scented geraniums are also commercially valued for their use in soaps, cosmetics, and perfumes (Saxena et al. 2000). In India, one particular species, Pelargonium graveolens, acts as the primary resource for geranium oil. Many cultivars of this plant are grown around the world for harvesting geranium oils, but the highest quality essential oil is produced by the 'Bourbon' cultivar, which originates from Reunion Island (Gupta et al. 2002). However, efforts to grow this 'Bourbon' cultivar in other parts of the world have been limited due to the plant's sensitivity to pests and disease. 
Use of plant tissue cultures has arisen as a way to enhance production and overcome obstacles associated with commercial propagation of scented geraniums, either for ornamental use or for essential oil production. Gupta et al. (2002) have achieved somaclonal variation through regeneration of plants via callus cultures. This approach was effective for increasing variability, leading to a wider genetic base from which desirable plant characteristics could be chosen. This study resulted in cultivation of a superior somaclone that produced unusually high yields of high quality geranium oil. Various other groups of researchers investigating $P$. graveolens, including Saxena et al. (2000) and Rao (1994), have also established efficient procedures for plant regeneration from callus, offering potential for improvement in plant characteristics and in commercial propagation.

\subsection{Formation and Applications of Nanoparticles}

Development and application of nanoparticles has emerged as an exciting new topic in modern technology. The unique optical, electrical, and magnetic properties of nanoscale materials have attracted much interest (Kim et al. 2002). These properties are greatly altered by changes in nanoparticle morphology, which ranges from spheres to cubes, rods, disks, wires, and triangles. Anisotropic particles have demonstrated complex physical properties in comparison to spherical nanoparticles. While nanospheres still have purpose in certain optical and biological applications, the anisotropic nanostructures have become the main focus of nanotechnology research, which seeks to understand and utilize these interesting properties (Murphy and Jana 2002). Manipulation of nanoparticle morphology and aspect ratios (the ratio of the lengths of the largest 
dimension to the smallest dimension) has led to an array of nanomaterials that catalyze different reactions. Work with nanorods and nanowires has revealed that these materials can emit polarized light and exhibit lasing. The goal of developing computer chips with nanoscale elements has further fueled research on these types of nanostructures.

The remarkable optical properties demonstrated by metal nanoparticles are directly related to the surface plasmon resonances they possess (Liz-Marzán 2004). Surface plasmon resonance (SPR) refers to the oscillation of conduction electrons that arises as a result of electromagnetic radiation. Metal nanoparticles that have free electrons have plasmon resonances that are caused by electromagnetic radiation in the visible spectrum. Since silver and gold (along with copper and alkali metal) nanoparticles possess free electrons (due to their nanoscale dimensions), they exhibit unique intense colors, and the SPR bands of these materials can be detected using spectrophotometry over the visible wavelengths of light.

SPR exhibited by isotropic particles, such as spheres, differs significantly from that of anisotropic particles. While nanospheres typically have plasmon resonance oscillations at one specific wavelength, anisotropic particles have both transverse and longitudinal oscillations that occur at different wavelengths of light. Therefore, in spectra for anisotropic particles, two separate SPR bands will be detected. On the other hand, particles such as spheres will display only a single peak in their spectra.

The colors displayed by these metal nanoparticles are contingent upon many factors besides composition (Liz-Marzán 2004). The shape and size of these particles have substantial influence on the color, and only a slight variation in geometry can cause significant color changes, which are reflected in the spectra. As mentioned before, the 
spectra of anisotropic particles such as nanorods will show two distinct peaks, corresponding to transverse and longitudinal oscillations. When the aspect ratio of the nanoparticles is altered even slightly, the longitudinal electron oscillation is greatly affected, which can cause striking color changes. The influence of the surrounding medium on the nanoparticles must also be considered, and the refractive index of the material in which the nanoparticles are dispersed helps determine the optical properties. It has also been found that the interaction between neighboring nanoparticles affects the resonance wavelength, and when nanoparticles are very close together, the models describing the behavior of individual particles are no longer applicable.

\subsubsection{Current and Future Uses of Gold and Silver Nanoparticles}

Applications for gold and silver nanoparticles are quickly increasing in number. In fact, according to Bourne Research, silver nanoparticles is one of the fastest growing product categories in the nanotechnology industry (AZoNano.com news ID 2172, 2006). Several products that incorporate silver nanoparticles into their design have already entered the consumer market. The antimicrobial property of silver, which is enhanced in nanoparticles due to an increase in surface area, has been exploited to improve the function of washing machines, refrigerators, filters, and food containers (AZoNano.com news ID 2172, 2006). Manufacturers of products like footwear and pillows have also turned to silver nanoparticles in an attempt to create antimicrobial merchandise that is healthy and odor-free.

The health field, too, has discovered several promising possibilities for silver nanoparticle applications. Textiles embedded with these antimicrobial particles could 
result in a much cleaner environment for hospitals and clinics (AZoNano.com news ID $2172,2006)$, where people are more susceptible to infections. Furthermore, in recent research, silver nanoparticles have also demonstrated the ability to attack viruses, such as HIV-1, to possibly prevent transmission and kill infected cells (Elechiguerra et al. 2005). In this instance, the nanoparticles exhibit size-dependent preferential binding to the viral envelope, which in turn inhibits binding of the virus to host cells. Consideration has been given to the role of silver nanoparticles in treatment of other forms of illness. For example, research has indicated that nanosize silver is highly toxic to bacteria associated with cystic fibrosis (AZoNano.com news ID 2050, 2006).

In addition, investigation of gold nanoparticles has led to several important technological advances (Shankar et al. 2005). They have been recognized as effective tools for development of sensors, and also have applications in catalysis. Because gold nanotriangles show evidence of NIR (near infrared) absorption, they can be used in development of optical coatings that provide a means of energy-efficient relief. When these thin films of nanotriangles are coated onto glass, transmission of heat through the glass is significantly reduced. This coating could be applied to windows in regions of high temperatures. Another exciting potential of these gold nanostructures, again with respect to gold's NIR absorption capabilities, is to cause hypothermia of cancer cells.

\subsubsection{Potential Harms of Nanotechnology}

Despite all the praise nanotechnology has received for its possible contributions to science and medicine, health and environmental effects of nanoparticles have become growing concerns. Inadequate research has been conducted on many nanomaterial 
products. Many nanoparticles are regarded with safety precautions identical to those that apply to the bulk material (Colvin 2003), even though these materials have unique properties due to their size. Toxicity studies have been carried out for some types of nanoparticles, but little is known about harmful effects of most types of nanoparticles, including those comprised of silver and gold (Mitchell et al. 2004). However, since most engineered nanoparticles are manufactured in liquid media, exposure through inhalation of nanoparticles may not be a great concern (Colvin 2003). More research is needed to assess the effects nanoparticle processing and use may have, not only on workers, but also on the ecosystem, since an increase in the production of these materials will likely lead to their release into water and soil (Colvin 2003).

\subsubsection{Biosynthesis of Silver and Gold Nanoparticles}

Synthesis of nanoparticles has typically been carried out by chemical methods, which involve using toxic compounds. Only recently has synthesis by biological methods been explored and developed. Biosynthesis of silver and gold nanoparticles is favored for its low environmental impact.

Processes employing microorganisms, such as bacteria, actinomycetes (a special classification of bacteria), and fungi, to create silver and gold nanoparticles, have been studied for some time (Shankar et al. 2003b). For example, silver nanoparticles having monodispersed shape and size have been produced using Pseudomonas stutzeri AG259, a bacterium found in silver mines. Extracellular formation of gold nanoparticles by the actinomycete Thermomonospora sp. has also been observed. While these and other methods using microorganisms continue to be pursued, plants are now being recognized 
as having potential for economically viable and environmentally sound nanoparticle production. Both intracellular and extracellular syntheses of nanoparticles have been investigated using various forms of plants and plant extracts.

\subsubsection{Synthesis by Plant Systems}

\subsection{Intracellular Synthesis by Plant Systems}

Intracellular synthesis of both silver and gold nanoparticles by living plants has been achieved by Gardea-Torresdey and co-workers (1998, 2002, 2003). They have observed that alfalfa (Medicago sativa) sprouts grown in gold-rich agar media actively uptake $\mathrm{Au}^{\mathrm{O}}$ from the media and accumulate nanoparticles in their tissues. The $\mathrm{Au}^{3+}$ is reduced to $\mathrm{Au}^{\mathrm{o}}$ in the solid media and the latter is uptaken by the living plants. The same phenomenon has occurred with silver; $\mathrm{Ag}^{+}$is converted to $\mathrm{Ag}^{0}$ in solid media, and the atoms are absorbed by the alfalfa plant and then assembled into organized nanoparticles ranging from 2-20 $\mathrm{nm}$ in diameter. For cases of both gold and silver, the nanoparticles are transported into the plant via roots and are carried to shoots.

\subsection{Extracellular Synthesis by Plant Systems}

Extracellular synthesis of silver and gold nanoparticles has been accomplished using the extract of several species of plants. Different compounds extracted from aloe (Aloe vera), lemongrass (Cymbopogon flexuosus), neem (Azadirachta indica), and tamarind all show propensities for metal nanoparticle formation. Extracts from aloe (Chandran et al. 2006), lemongrass (Shankar et al. 2004c), and tamarind (Ankamwar et 
al. 2005) have all been shown to play roles in the reduction of $\mathrm{Au}^{3+}$ to form $\mathrm{Au}^{\circ}$ flat crystalline nanotriangles. Aloe readily transforms $\mathrm{Ag}^{+}$ions into nanoparticles as well, though only spherical particles are formed (Chandran et al. 2006). Shankar et al. (2004b) also reported on production of nanoparticles using neem leaf extract. Neem possesses the ability to reduce both silver and gold to form polydisperse nanostructures. Interestingly, when both $\mathrm{Au}^{3+}$ and $\mathrm{Ag}^{+}$ions are competitively placed in solution, neem will assemble nanoparticles composed of a gold core and a silver shell.

Extract from scented geraniums has also displayed a capability for assembling nanoparticles. Nanoparticle formation using this approach is the primary focus in the research conducted for this thesis. Shankar et al. (2003b) performed experiments using extract made from leaves of the geranium Pelargonium graveolens (cultivar was not specified) to reduce $\mathrm{Ag}^{+}$to $\mathrm{Ag}^{\mathrm{o}}$ crystalline nanoparticles. They observed mainly spherical nanoparticles ranging from 16-40 $\mathrm{nm}$ that remained very stable even after 4 weeks. In a parallel study, Shankar et al. (2003a) achieved reduction by geranium leaf extract to form gold nanoparticles. This procedure led to development of stable gold nanoparticles with great variability in size and morphology. In this case, particles displayed a number of shapes including rods, flat disks, and triangles. In addition, Shankar and his colleagues (2004a) later conducted follow-up studies to examine the effects of using different parts of the geranium plant for preparation of extract. Experimentation with extract of stems and roots uncovered more exciting findings, one being a significant decrease in reaction times. Among leaf, stem, and root extracts, root extract exhibited the fastest reaction for reduction of silver ions, while stem extract showed the fastest rate for reduction of gold 
ions. In each case, the reaction rate observed was greatly accelerated compared to previous studies using fungi or bacteria to carry out the reduction.

While various compounds present in the geranium extract could participate in the reaction, it is suspected that terpenoids or alkaloids are responsible for the reduction and capping of the molecules (Shankar et al. 2004a). Whatever the compounds may be, FTIR spectra have indicated that the capping molecules are the same for Ag nanoparticles produced by either stem or root extract. The biomolecules that cap Au nanoparticles appear to be different from those that cap Ag, and also appear to be different in the cases of stem and root extract.

Although nanoparticle formation directed by molecules found in geranium extract has been reported in recent years, much exploration remains to be done in order to provide a better understanding of this complex bio-process. In addition, more improvements must be made to further reduce reaction times and to provide shape and size control before biosynthetic pathways can compete with chemical methods (Shankar et al. 2004a). Part of the research conducted for this thesis involves further exploratory study of this bio-reaction involving geranium extract. It is reasonable to believe that the reactions are cultivar-dependent. The three cultivars of Pelargonium graveolens we used are 'Rose', 'Rober's Lemon Rose', and 'Lady Plymouth', whose uses in similar studies have not been reported in the literature. 


\section{Chapter 3. Objectives}

The aim of this research is to provide a better understanding of the uptake of silver and gold ions from aqueous solution into plant systems and the formation of nanoparticles therein. Plants in different forms, including sprouts, suspended cells, and hairy roots, are involved, and modern plant technologies are employed. Specifically, the objectives of this research are as follows:

1. To induce and maintain callus cultures for Catharanthus roseus, Stevia rebaudiana, and three cultivars of Pelargonium graveolens, and to continue subculturing for Beta vulgaris, which was previously established.

2. To produce cell suspension cultures for Catharanthus roseus, Stevia rebaudiana, Beta vulgaris, and Pelargonium graveolens, as well as hairy root cultures for Stevia rebaudiana, and use them to study uptake of silver and gold ions from solution to provide insight into intracellular formation of silver and gold nanoparticles in those plant cells.

3. To examine the ability for alfalfa (Medicago sativa) and mung bean (Vigna radiata) sprouts to uptake silver ions from solution, and to assess the potential of producing silver nanoparticles from those sprouts.

4. To study extracellular formations of silver and gold nanoparticles by three cultivars of Pelargonium graveolens, and to investigate the factors involved in their formation. 


\section{Chapter 4. Materials and Methods}

\subsection{Callus Cultures}

The following subsections provide a brief account of procedures used in this study. All callus cultures were established and subcultured using aseptic techniques (Appendix A), and were placed on solid agar media in glass baby jars or petri dishes. Detailed information for media preparation and establishment and maintenance of callus for each species can be found in Appendices B and C.

\subsubsection{Catharanthus roseus}

Callus culture of Catharanthus roseus has been established from the leaves of plants grown in Room 441 ESB, and has been subcultured now for almost 2 years. Cultures are contained in an incubator that keeps the temperature close to $25^{\circ} \mathrm{C}$ and has cycles of 16 hours fluorescent illumination followed by 8 hours dark. Subcultures are performed every 2-3 weeks. All procedures were adopted from Beach (1998) with slight modifications.

\subsubsection{Stevia rebaudiana}

Stevia rebaudiana callus cultures were initiated starting from plantlets grown from seeds (Geo. W. Park Seed Co., Inc.) according to methods described by Swanson et al. (1992) and have been growing under constant fluorescent lighted conditions. A warm water bath keeps culture temperatures at $27{ }^{\circ} \mathrm{C}$. Subcultures were required at approximately 4-week intervals. 


\subsubsection{Beta vulgaris}

Beta vulgaris cultures were previously established from plantlets grown from seeds (W. Atlee Burpee and Co.), and subcultures have been carried out over the course of this study. Environmental conditions were identical to those of C. roseus, though media were quite different. Subcultures were conducted every 4-5 weeks. Details on media preparation and required growth conditions are given in Appendix B (Beach 1998).

\subsubsection{Pelargonium graveolens}

After several failed attempts to begin callus culture for three cultivars of Pelargonium graveolens - 'Lady Plymouth' (LP), 'Rose' (R), and 'Rober's Lemon Rose' (RR), finally callus formation was observed using two separate methods. One method that worked was derived from Rao (1994). It involved using leaf explants to induce formation on MS medium (designated MS(a) for convenience). Shortly after induction was attempted, the leaf explants turned brown and appeared to be dying. Not until over $2 \frac{1}{2}$ months later were calli detected. The other procedure, which used stem segments inoculated vertically, was given by Saxena et al. (2000). This method used a different variation of MS media, designated MS(b) from here on out. This method did not produce results as quickly as indicated by Saxena et al. (2000), but callus did develop after 3 weeks. Pieces of each type of callus (each cultivar on each medium) were then subcultured onto both types of media (MS(a) and MS(b)). For each method, the callus growth remained slow, and many cultures did not survive the first subculture. 
Except for one jar, all callus developed from stem explants became contaminated upon subculturing, both to MS(a) and MS(b) media. The one remaining jar containing callus derived from stem explants is maintained on MS(b) medium. It never became contaminated, but the callus started to differentiate into roots and shoots, and tiny plantlets have begun to grow. This indicates that the balance of growth hormones is not sufficient for maintaining undifferentiated callus.

For callus that was initiated from leaf explants but was later transferred from MS(a) medium to MS(b) medium, the same phenomena has been observed, and tiny roots and shoots have developed. More success was had with callus that developed from leaves but was initiated and maintained only on MS(a) medium. For this situation, both $\mathrm{R}$ and LP callus are viable, and growth is evident, especially for callus from LP. However, the morphology of this callus is globular and inconsistent, suggesting that perhaps the growth hormones for MS(a) medium are also not properly formulated for maintenance of callus.

Another attempt was made to induce geranium callus using stem explants, since the callus that developed using this method looked healthier and more uniform than the callus that developed from the leaf explants. Callus again appeared after about 3 weeks, and it remains to be subcultured.

\subsection{Suspension Cultures}

For suspension cultures, the medium formulation for each species is identical to that for the corresponding callus, only with agar omitted. See Appendix D for a more thorough explanation of methods used for induction and maintenance of suspension 
cultures. Procedures for addition of $\mathrm{AgNO}_{3}$ to the medium are detailed in Appendices $\mathrm{E}$ and F, and preparation of samples for AA analysis is covered in Appendix I.

\subsubsection{Catharanthus roseus}

Suspension cell cultures of $C$. roseus were established, as described by Bayraktar (1996). The Erlenmeyer flasks $(500 \mathrm{ml})$ containing the suspension were agitated on an orbital shaker at 110-120 rpm to allow for maximum nutrient uptake by cells. Flasks were kept at $25 \pm 1{ }^{\circ} \mathrm{C}$, with a photoperiod of $16 \mathrm{hrs} \operatorname{light} / 8 \mathrm{hrs}$ dark. Subcultures were performed every 2 weeks.

\subsubsection{Stevia rebaudiana}

Suspension cultures of S. rebaudiana were also successfully established from callus. Erlenmeyer flasks $(500 \mathrm{ml})$ were kept under $24 \mathrm{hr}$ fluorescent light on an orbital shaker at $110-120 \mathrm{rpm}$. The ambient temperature was $27 \pm 1^{\circ} \mathrm{C}$. Subcultures were conducted every 2 weeks. A portion of the suspension was added to fresh MS medium with a ratio of approximately 1:7 (suspension:fresh medium).

Stevia suspensions were tested for uptake of silver from solution. Upon addition of $\mathrm{AgNO}_{3}$ to the liquid MS medium, $\mathrm{Ag}^{+}$ions became free to interact with the cells. For optimum results, $\mathrm{AgNO}_{3}$ must be added to suspension cultures when cells are most active, which is during their exponential growth phase. Therefore, silver solution was added in the middle of the subculture period. For Stevia suspension, the subculture period is 14 days, and thus, the silver solution was introduced on day 7. AA analysis has shown evidence that during the incubation period of 7 days, silver ions are transported 
into the cells. At the end of the period, cells and contents were washed six times with $200 \mathrm{ml}$ aliquots of distilled/deionized water, all under vacuum filtration. Then they were dried overnight at $80^{\circ} \mathrm{C}$, burned for 3 hours at $700^{\circ} \mathrm{C}$, and dissolved in concentrated nitric acid. All $\mathrm{Ag}^{\mathrm{o}}$ particles or $\mathrm{Ag}^{+}$ions present inside the cells were also dissolved by the acid, and could be readily detected by AA. A Perkin-Elmer 2380 Atomic Absorption Spectrophotometer was used for analyzing samples. Several concentrations of $\mathrm{Ag}^{+}$ solution, ranging from $0 \mathrm{ppm}$ to $40 \mathrm{ppm}$, were tested for uptake by cells.

\subsubsection{Beta vulgaris}

Suspension of red beet was similarly developed from callus. Temperature and light conditions were identical to $C$. roseus; suspensions were maintained at $25 \pm 1^{\circ} \mathrm{C}$ with

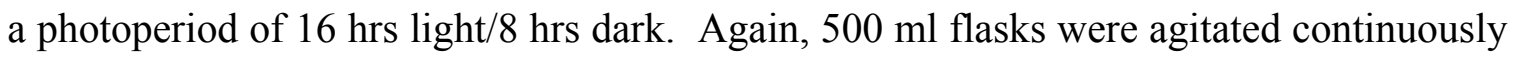
on a shaker at 110-120 rpm. Subcultures were carried out every 2 weeks by transferring approximately $25 \mathrm{ml}$ of suspension to $175 \mathrm{ml}$ fresh liquid A2 medium.

\subsection{Hairy Root Cultures}

Details of the experimental procedures related to the following two subsections can be found in Appendix $\mathrm{H}$.

\subsubsection{Catharanthus roseus}

Using procedures outlined by Bayraktar (1996), attempts were made to induce hairy root formation in sterile C. roseus plantlets. Sterile plantlets were developed from seeds in small jars containing solid B5 medium. After germination, they were placed in 
an incubator which kept temperatures at $25 \pm 1^{\circ} \mathrm{C}$, and had a 12 hour photoperiod. After about 6 weeks, when plants were healthy and strong, small scratches were made on the stems of the plantlets with a sterile sewing needle, and small drops of Agrobacterium rhizogenes (ATCC 15834) were squirted onto the wounds. Plantlets were kept in darkness overnight to prevent bacteria from drying up. From this point on, the infected plantlets were again exposed to light for 12 hours per day. Fuzzy root-like structures appeared from the sites of injury, which are believed to be hairy roots. After several weeks, these structures were removed from the plantlet and were transferred to fresh $1 / 2$ B5 medium containing ampicillin. After this subculture was performed, no growth was noticeable, and cultures became necrotic. No further attempts were made, due to limit of time.

\subsubsection{Stevia rebaudiana}

Hairy root cultures of Stevia have been accomplished through injection of sterile plantlets with Agrobacterium rhizogenes (ATCC 15834). Plantlets were grown from seeds on $1 / 2 \mathrm{MS}$ medium under $12 \mathrm{hr}$ fluorescent light at $25 \pm 1^{\circ} \mathrm{C}$. Plants were strong and healthy, and stems were sufficiently thick after about 6 weeks. A hypodermic needle was used to minimize injury and to ensure precise placement of the bacteria under the surface of the epidermis (epidermal cells). Hairy roots emerged from injection sites after 1-2 weeks, and were subcultured onto solid $1 / 2$ MS medium with ampicillin to eliminate $A$. rhizogenes bacteria. After 3 subcultures, ampicillin was no longer used. All cultures were kept at $25 \pm 1^{\circ} \mathrm{C}$, and two different lighting specifications were examined. Some hairy root cultures were contained in a dark box to prevent any light from entering. 
Others were submitted to cycles of $16 \mathrm{hr}$ fluorescent light followed by $8 \mathrm{hr}$ dark. Subcultures were conducted every 3 weeks. This procedure for induction and maintenance of Stevia hairy roots was developed by Yamazaki and Flores (1991).

After the hairy root cultures had been successfully subcultured to media not containing ampicillin, they could be transferred to liquid media. Small pieces of the hairy roots were excised and placed into liquid $1 / 2$ MS medium. The cultures were then placed on orbital shakers at 110-120 rpm and were submitted to the same light conditions as they previously were exposed to on solid media. Some were placed in a dark incubator and others grew under $16 \mathrm{hr}$ light $/ 8 \mathrm{hr}$ dark cycles.

\subsection{Sprouts}

Two species of sprouts were studied to determine ability for the intact sprouts to facilitate Ag nanoparticle formation. Mung bean (Vigna radiata) sprouts and alfalfa (Medicago sativa) sprouts were grown in sprouters for a duration of 7 days, following a one day soaking period in which the seeds were submersed in a beaker of water. Concentrations of $\mathrm{Ag}^{+}$solution, varying from $0 \mathrm{ppm}$ to $80 \mathrm{ppm}$ initial silver concentration, were used in the water supply provided for the sprouts. In addition, two types of sprouters were used, both under $24 \mathrm{hr}$ fluorescent lighting at approximately 25 ${ }^{\circ} \mathrm{C}$. Automatic sprouters, hereafter designated as A-sprouters, hold the seeds on a screen that is periodically sprayed with the silver solution. The solution then drains through to the reservoir below, and is recirculated with a pump. This sprouter is made of dark tinted plastic, and, therefore, the sprouts are partially protected from light. The simultaneous sprouter, referred to as the S-sprouter, consists of a simple screen that sits in a shallow 
tray of solution. The screen is framed by sides that keep the sprouts confined, and a divider separates the screen into two equal parts that can accommodate two separate species. A clear plastic lid covers the tray, so full light can pass through. The S-sprouter ensures that the sprouts contact the solution only near the roots, as opposed to the Asprouter, which sprinkles solution over the entire plant. In the S-sprouter, pure distilled/deionized water was added gradually over the 7-day period in order to keep the appropriate water level and to prevent $\mathrm{Ag}^{+}$concentration from fluctuating significantly.

After a one-week growth period, sprouts were harvested. They were washed thoroughly, dried, and burned to ash. The ash, which includes any silver particles or ions that had accumulated inside the sprouts, was then dissolved in concentrated acid and diluted. AA analysis was used to find the concentration of silver retained inside the sprout plant cells. Appendix G presents further details on preparation of seeds, silver solution, and sprouters, and Appendix I explains how samples were made for AA analysis.

\subsection{Geranium Extract}

Various experiments were performed using geranium extract as a reactant. Initially, experimental procedures were based on methods developed by Shankar et al. $(2003 a, b)$. In this case, the extract was prepared using leaves of 3 cultivars of scented geranium (Pelargonium graveolens): 'Rose' (R), 'Rober's Lemon Rose' (RR), and 'Lady Plymouth' (LP). The leaves were ground finely, water was added, and the mixture was then boiled for a short period of time. Afterward, the leaves were separated from the solution and discarded, while small amounts of the extract (10 $\mathrm{ml}$ aliquots) were reacted with either 0.001 $\mathrm{M} \mathrm{AgNO}_{3}\left(107.9 \mathrm{ppm} \mathrm{Ag}{ }^{+}\right)$solution or 0.001 M KAuCl4 (196.97 ppm 
$\mathrm{Au}^{3+}$ ) solution. The reactions all took place in clean $125 \mathrm{ml}$ clear glass jars. Several variables were tested to find factors that influence the reaction. In some situations, these samples were continuously exposed to fluorescent lighting. Other samples were kept in darkness. Exposure to air was also altered to determine if oxygen had any role in the reaction. For $\mathrm{R}$ and $\mathrm{LP}$, the $\mathrm{pH}$ was also measured periodically over the course of the reaction.

Other experiments were conducted with modifications to the procedure to enable a deeper understanding of the reactions taking place. One such study examined the influence that boiling time had on the reaction by reacting samples of geranium leaf extract that had been prepared with boiling times ranging from 0 minutes (no exposure to heat) to 20 minutes boiling. We also investigated the effect of boiling extract after leaves had been filtered out with Whatman \#1 filter paper (pore size of $11 \mu \mathrm{m}$ ). Therefore, most of the cell membrane would presumably be filtered out and could no longer influence the composition of the extract. Further studies involved examining the progress of the reaction in a $\mathrm{pH}$-controlled environment. For these reactions, the $\mathrm{pH}$ was adjusted immediately after reactants were mixed together, and the resulting products were analyzed. Initial $\mathrm{pH}$ values used range from 3.5 to 6.0. These modified procedures have been performed only with $\mathrm{KAuCl}_{4}$. Specific details of procedures used can be found in Appendix J.

For all the various experiments, a Spectronic Genesys 5 Spectrophotometer was used to analyze the spectra over the wavelengths of visible light. This served to help characterize what types of products may be forming and to indicate any discrepancy that was present due to alteration of variables involved in the reaction. 
The colors that evolved from these reactions and the spectra generated all indicate that silver or gold nanoparticles are being produced by reaction of either $\mathrm{AgNO}_{3}$ solution or $\mathrm{KAuCl}_{4}$ solution with geranium extract. To provide more evidence that these nanoparticles are present, the reactions were simulated chemically using $\mathrm{NaBH}_{4}$ to reduce the silver or gold ions to form nanoparticles in solution. The reduction was performed in the presence of trisodium citrate, which acts as a capping agent to stabilize the nanoparticles. This chemical reduction allowed for comparison between the nanoparticle solutions produced chemically and those produced using geranium extract. The procedures for the chemical syntheses of silver nanoparticles and gold nanoparticles were taken from Jana et al. (2001 a, b). 


\section{Chapter 5. Results and Discussion}

\subsection{Callus Cultures}

Callus cultures of Catharanthus roseus (periwinkle) and Stevia rebaudiana (stevia) have all been successfully established and maintained for over one year. Callus of Beta vulgaris (red beet) had been previously established and subcultures were continued for use in this project. Each of the three species has been affected by episodes of microbial contamination to a part of the callus, severely hindering growth. However, through careful subculturing, this problem has been overcome and all cultures have returned to a healthy state. Callus culture for the three cultivars ('Rose', 'Rober's Lemon Rose', and 'Lady Plymouth') of Pelargonium graveolens (scented geraniums) were also initiated, after much effort. Some of the callus culture endured the first subculture, but many became contaminated and died. For those cultures that remain, the future outlook is not certain. Those growing on MS(a) medium are globular in appearance, and growth is slow. On the other hand, callus cultures maintained on $\mathrm{MS}(\mathrm{b})$ medium are differentiating into roots and shoots. Both situations indicate that the amount or ratio of cytokinin and auxin growth hormones as given by Rao (1994) and Saxena et al. (2000) is not appropriate for maintaining undifferentiated callus cultures for these particular cultivars of scented geranium.

Dedifferentiated callus cultures are often necessary prerequisites for producing other types of cultures. In this study, callus was developed with the intention of creating suspension cell cultures. Even after suspension cultures were induced, subculturing of callus was continued as a precautionary measure in case suspension cultures become 
contaminated or die. Photographs of callus can be found in Figure 5.1, which displays images of periwinkle, stevia, red beet, and geranium. It can be seen that the callus cultures of periwinkle, stevia, and red beet shown in Figure 5.1 have been in the maintenance stage for a period of time. However, the geranium callus in Figure 5.1 is still in the initiation phase, and the explant is pictured along with the callus. The stem is horizontally oriented still with a leaflet attached, and the vertical green mass larger in size than the stem is the callus that has recently emerged.

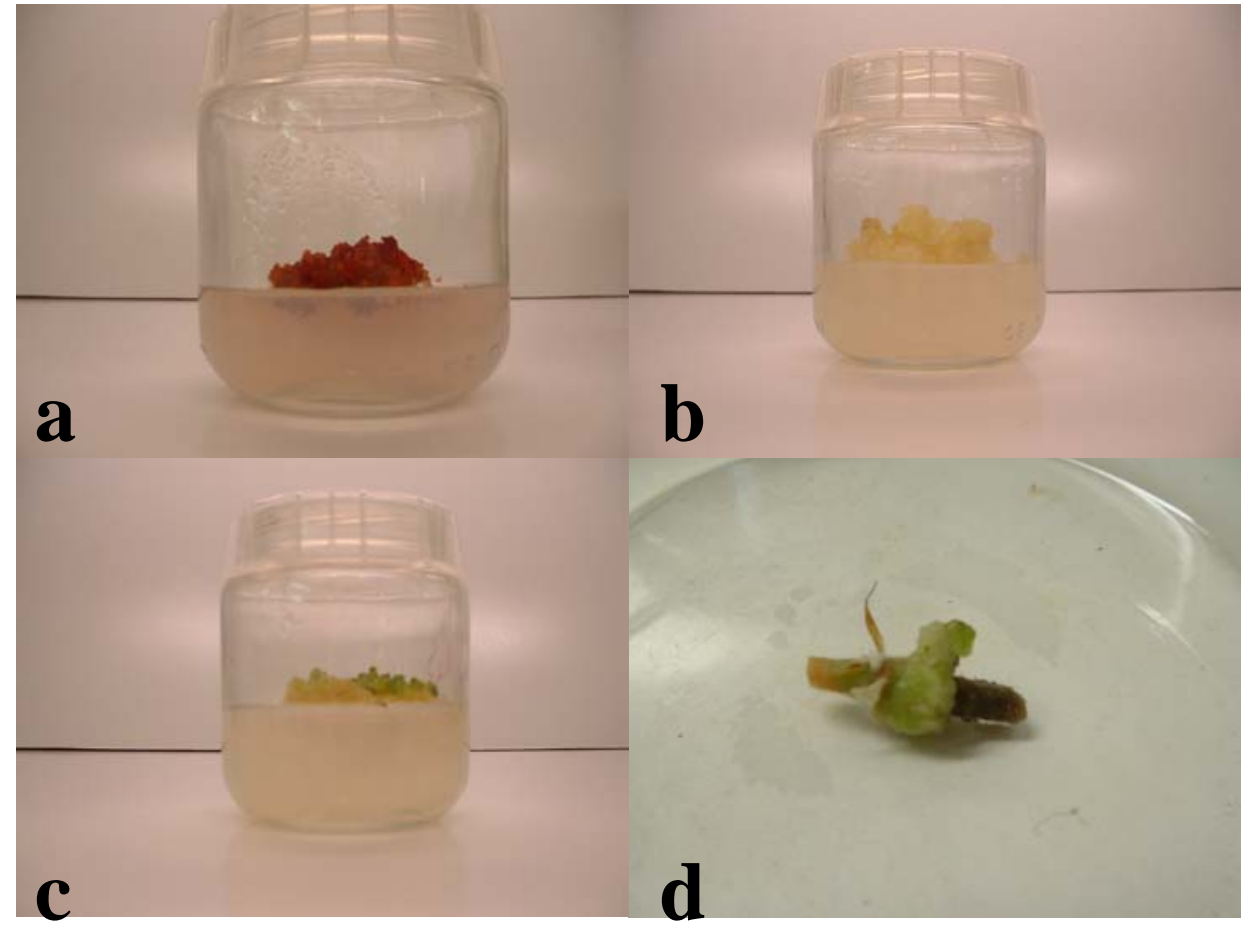

Figure 5.1. Callus cultures of (a) red beet, (b) periwinkle, (c) stevia, and (d) scented geranium. 


\subsection{Suspension Cultures}

Suspension cell cultures of S. rebaudiana, C. roseus, and B. vulgaris were attained from callus. S. rebaudiana suspension cultures have been studied for intracellular accumulation of silver and gold from solution. Use of suspension cultures provides a simple and consistent means of observing basic cell functions in a controlled environment.

Stevia suspensions were tested for uptake of silver from solution. When $\mathrm{AgNO}_{3}$ solution is added to the liquid MS medium, $\mathrm{Ag}^{+}$ions are free to interact with the cells. AA analysis has shown evidence that during the exposure period of 7 days, silver is accumulated inside the cells, either in ion form or as elemental state particles. It is speculated that nanoparticle formation occurs upon cellular uptake of the metal ions into the vacuoles of the plant cells, but this has not been confirmed in the present study. At harvest, cells were washed very thoroughly to get rid of any extracellular sources of silver. When samples were dissolved in concentrated nitric acid, any silver present inside the Stevia cells was also dissolved by the acid, and could be readily detected by AA.

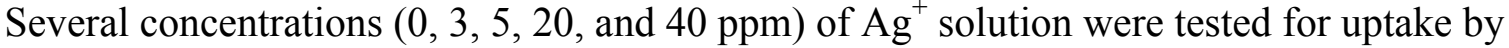
cells.

The initial concentrations of $\mathrm{Ag}^{+}$in the medium and the loading in the cells are shown in Figure 5.2. A blank solution free of $\mathrm{Ag}^{+}$was chosen to serve as a control. At the initial $\mathrm{Ag}^{+}$concentrations of $20 \mathrm{ppm}$ and $40 \mathrm{ppm}$, the toxic effect of silver on the plant cells was obvious from the change in appearance of cultures. The suspension is pale yellow in color at a healthy state, but became very dark-colored after addition of silver, indicating that the cells were killed by contact with silver. 
It was attempted to determine a threshold value below which cells can survive upon exposure to silver. Exposure to silver was also toxic to alfalfa and mung bean sprouts (see Section 5.4), and sprout growth was retarded even at an $\mathrm{Ag}^{+}$concentration of $10 \mathrm{ppm}$. Since cells in suspension cultures have little protection from their environment, the threshold concentration for suspension cultures should be much lower than that for similar experiments using sprouts. Therefore, $\mathrm{Ag}^{+}$concentrations of $3 \mathrm{ppm}$ and $5 \mathrm{ppm}$ were chosen for subsequent runs with Stevia suspension. However, these concentrations of $\mathrm{Ag}^{+}$were also damaging to the cells, causing a high percentage of cells to die. Because silver was so toxic to the unprotected cells, it is likely that the uptake of silver into the dead cells occurs mostly due to passive diffusion. Although cell death was observed for all tested concentrations, the curve seen in Figure 5.2 is slightly suggestive of some growth when initial silver concentrations are $5 \mathrm{ppm}$ or below. Since one of the focuses of Section 5.2 is to assess the possibility of forming nanoparticles using living plant cells, for silver nanoparticles this approach may not be worth pursuing further. However, for gold nanoparticles, the assessment is entirely different.

Similar experiments on Stevia suspension cultures were conducted with gold solution made with $\mathrm{KAuCl}_{4}$. Although data is limited, it is worth stating that for these studies with gold, no toxic response was observed. Cell samples were again analyzed by AA to determine whether $\mathrm{Au}^{3+}$ ions were indeed taken up into the plant cells. Figure 5.3 displays the results from these tests. By comparing Figures 5.2 and 5.3, it can be seen that for a particular metal concentration, the loading of gold in Stevia cells is slightly higher than loading of silver. Since it appears that gold has no significant negative effect on the cells, additional effort may be worthwhile. 




Figure 5.2. Dependence of silver loading in Stevia cells on initial silver concentration in suspension culture media.



Figure 5.3. Dependence of gold loading in Stevia cells on initial gold concentration in suspension culture media. 


\subsection{Hairy Root Cultures}

Genetic stability and fast growth rate of hairy root cultures are the main advantages of using this type of culture. Hairy roots were initially grown on solid media, but could later be transferred to liquid media. In this research, hairy root cultures were developed for use in reactions similar to those involving suspension cultures. Hairy root cultures maintained in liquid medium could be studied for metal ion uptake and nanoparticle formation, producing fast and reproducible results.

\subsubsection{Catharanthus roseus}

Attempts were made to induce hairy root formation in C. roseus, but yielded no useful results. Fuzzy, root-like projections, believed to be hairy roots, initially developed from sites that had been infected with Agrobacterium rhizogenes, but did not survive after the first subculture. When hairy root segments were transferred from the wound sites on the plantlet to medium containing an antibiotic, no growth took place and hairy root segments became necrotic. This was probably because too much time had passed before the hairy roots were excised and placed onto fresh medium, and they were not strong enough to survive and grow in the presence of an antibiotic. Thus, efforts were discontinued and turned to Stevia due to time constraints.

\subsubsection{Stevia rebaudiana}

Hairy roots of Stevia have been successfully developed and subcultured several times onto solid medium containing ampicillin followed by subcultures to solid medium free of ampicillin. Attempts were made to reduce the number of subcultures to media 
containing antibiotics. Instead of subculturing to $1 / 2 \mathrm{MS}$ with ampicillin three times as suggested by Yamazaki and Flores (1991), some cultures were transferred to $1 / 2$ MS without ampicillin after only one subculture. However, these attempts proved to be futile, since bacterial contamination, presumably due to A. rhizogenes, was still observed in these hairy root cultures. Hence, only hairy roots subcultured to antibiotic medium a minimum of three times were considered suitable for subculturing to medium that was free of antibiotics.

Later, a period of infection from a different contaminant delayed the transfer of the hairy roots to liquid media, but this was eventually resolved. Cultures have since recovered and are growing rapidly, and were eventually transferred to liquid media. Despite this change in growth environment, there is no sign of contamination or shock. Growth is evident even though growth hormones are not used.

Interestingly, throughout all stages of subculturing to various media, cultures grown in the dark exhibit faster growth rates and "hairier" roots than those exposed to light. Pictures of hairy root cultures grown on solid media, both in light and dark, are shown in Figure 5.4. Although hairy roots are genetically transformed and are not really roots, they may have properties similar to roots, which typically grow in darkness underground. This may explain why hairy roots grow more rapidly in darkened conditions.

Figure 5.5 shows the cultures 8 days after hairy roots were subcultured from solid to liquid media. Only small portions of the hairy roots were transferred to the flask, and we have observed rapid growth. 


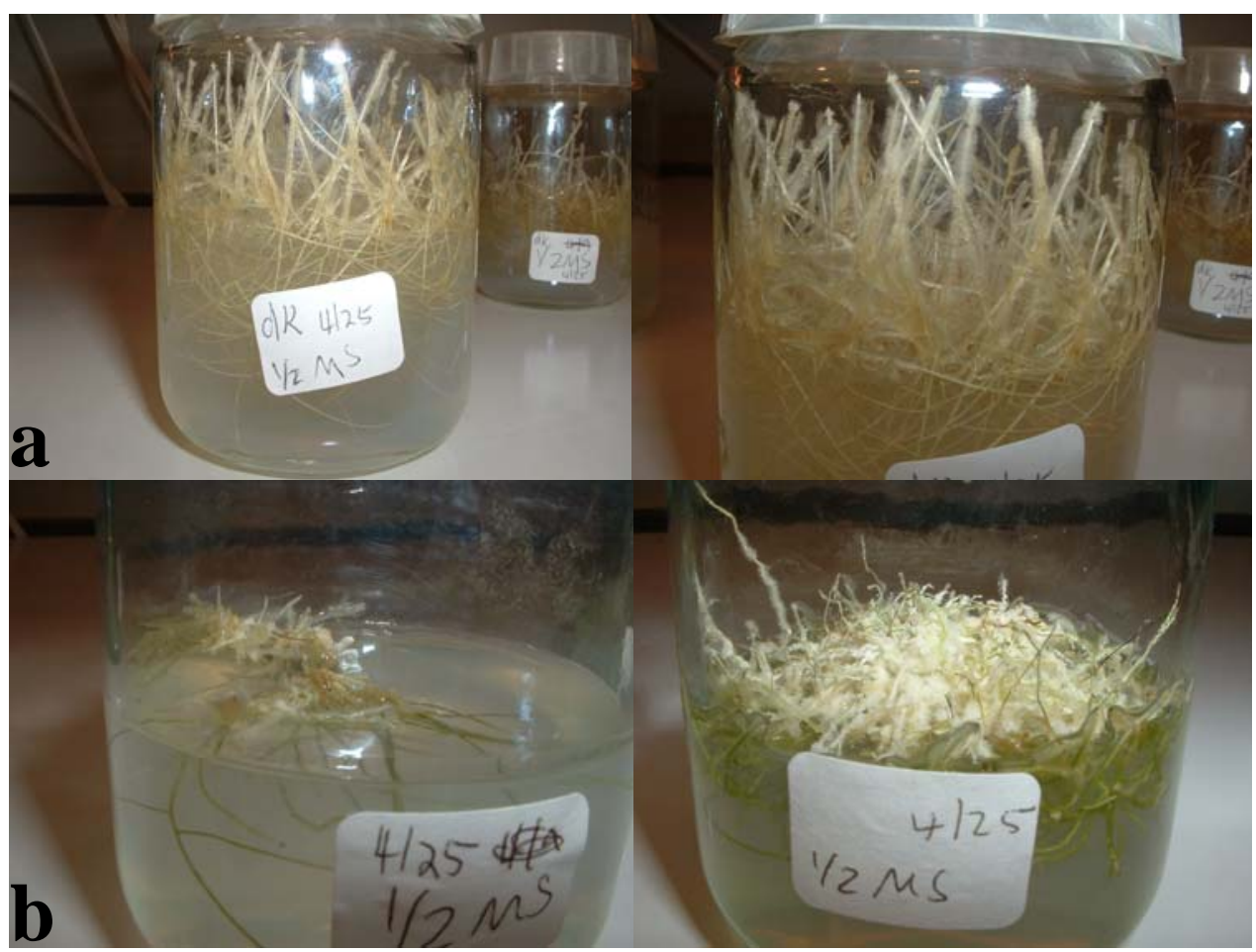

Figure 5.4. Hairy root cultures of Stevia 5 weeks after first subculture (a) growing in darkness, (b) grown with $18 \mathrm{hr}$ photoperiod.

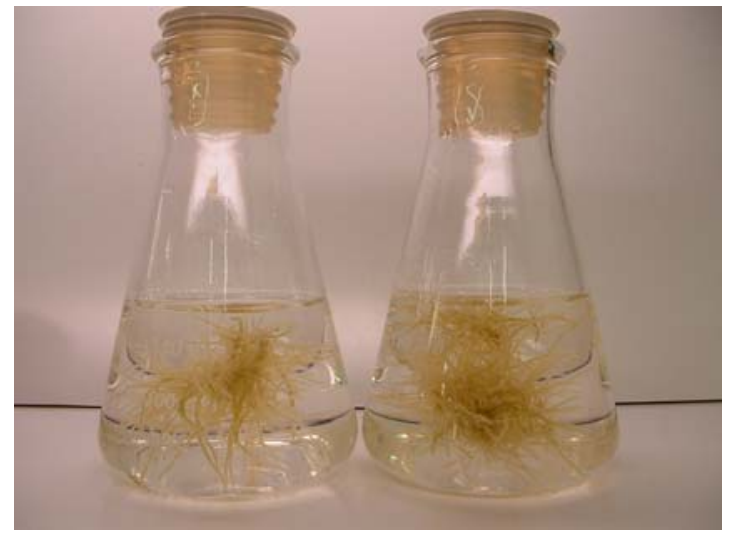

Figure 5.5. Stevia hairy root cultures grown under $18 \mathrm{hr}$ light, 8 days after subculturing from solid media to liquid media. 


\subsection{Sprouts}

Two species of sprouts were studied to determine the ability of intact sprouts to uptake silver from solution. Mung bean (Vigna radiata) sprouts and alfalfa (Medicago sativa) sprouts were grown for a week in sprouters containing $\mathrm{Ag}^{+}$solution. Despite the toxic effects seen in sprouts in response to $\mathrm{Ag}^{+}$and $\mathrm{Ag}^{\mathrm{o}}$, ability of sprouts to uptake silver was observed. AA analysis has verified the occurrence of this process with both mung bean and alfalfa. Results are displayed in Figures 5.6 and 5.7.



Figure 5.6. Dependence of silver loading in mung bean and alfalfa sprouts on initial silver concentration in sprouter solutions for type A-sprouter. 


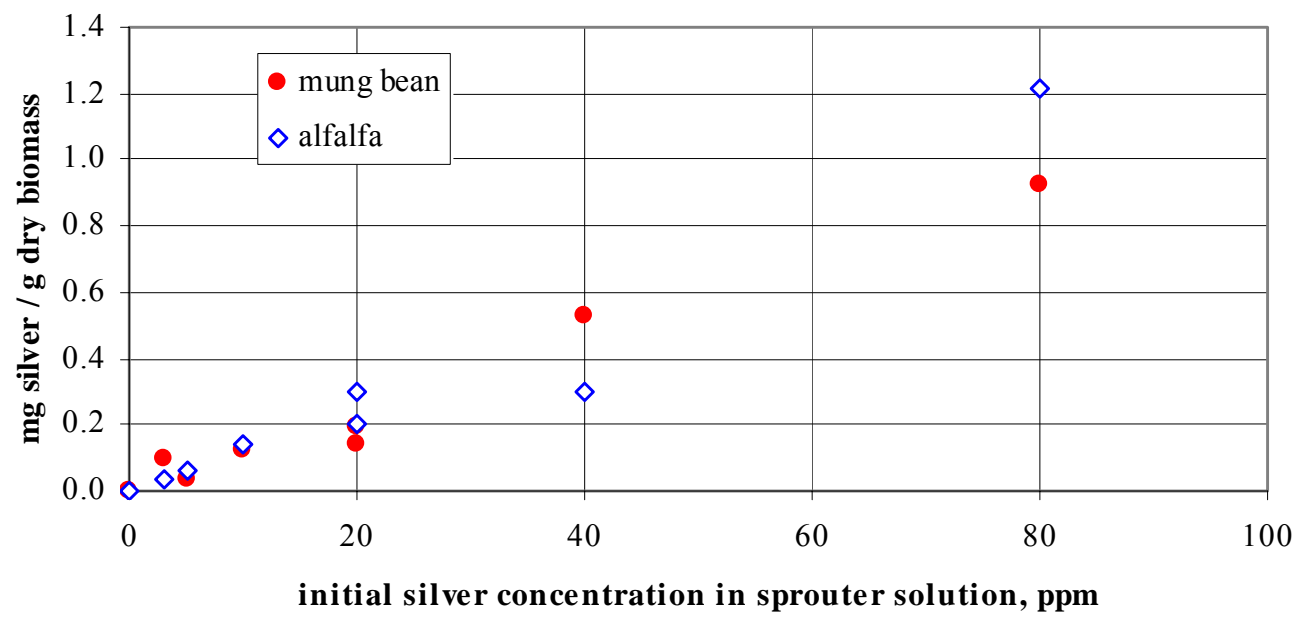

Figure 5.7. Dependence of silver loading in mung bean and alfalfa sprouts on initial silver concentration in sprouter solutions for type S-sprouter.

It must be acknowledged that, as seen in experiments with Stevia suspension, toxicity of silver to sprouts has had an effect on results. Figure 5.8 shows alfalfa sprouts grown in the A-sprouter with $20 \mathrm{ppm} \mathrm{Ag}^{+}$solution. It is clear from the photo that at this concentration, silver has detrimental effects on sprout germination and growth. The seeds located in the center of the sprouter are not fully doused with the solution, unlike the seeds placed on the periphery. Consequently, the growth toward the middle is much higher. At harvest, all of the seeds and sprouts were collected and mixed uniformly. Representative samples were collected from the mixture of sprouts and seeds that had not germinated. 


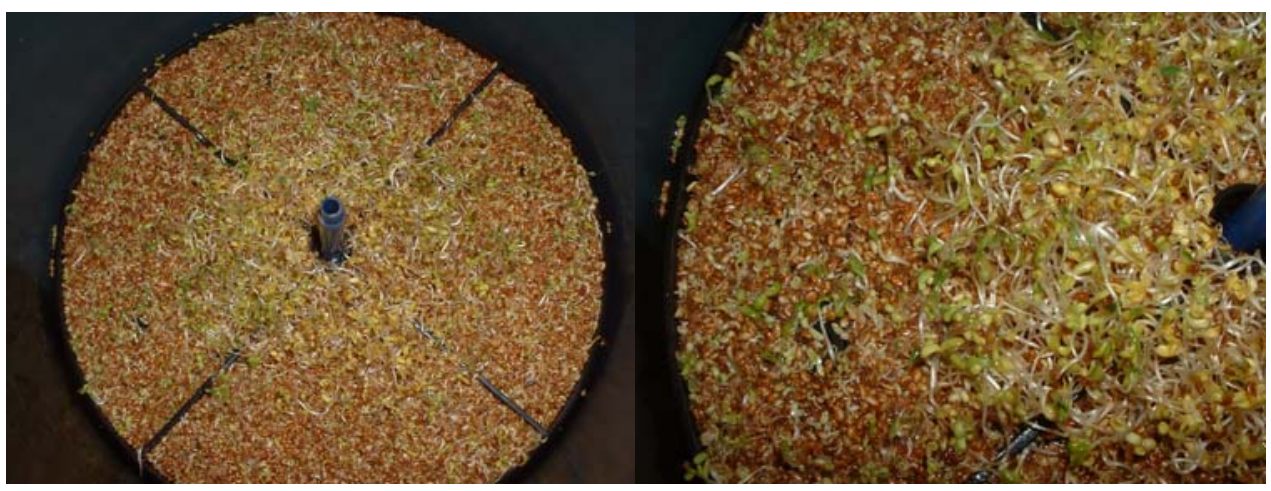

Figure 5.8. Alfalfa sprouts grown in A-sprouter for 7 days with $20 \mathrm{ppm}$ $\mathrm{Ag}^{+}$solution.

Pertaining to the A-sprouter, even when grown in solution with a concentration of 5 ppm $\mathrm{Ag}^{+}$, both alfalfa and mung bean were adversely affected, and still many seeds did not germinate. At this concentration, approximately half of the seeds grew into sprouts, but sprout growth was stunted. Thus, the results shown in Figure 5.6 do not accurately represent the accumulation of silver by the sprouts by active uptake. Since many of the seeds did not germinate and grow to form roots and shoots, it is likely that the presence of silver in the samples is at least partially due to passive diffusion. However, sprouts growing in the S-sprouter were not so strongly affected by exposure to silver, since the only point of contact of the sprouts with the solution was through the roots. At a concentration of $80 \mathrm{ppm} \mathrm{Ag}^{+}$, it was estimated that $40 \%$ of the mung bean seeds and $10 \%$ of the alfalfa seeds grew to produce leaves. At a concentration of $40 \mathrm{ppm} \mathrm{Ag}^{+}$, approximately $70 \%$ of the mung bean seeds and $50 \%$ of the alfalfa seeds grew enough to produce leaves, but sprouts appeared thin and unhealthy. Tests conducted with a silver concentration of $20 \mathrm{ppm}$ resulted in germination and growth of nearly all of the seeds. Therefore, the S-sprouter poses many advantages over the A-sprouter from a process standpoint. Sprouts grown in an S-sprouter can withstand exposure to higher 
concentrations of silver in solution. Furthermore, the S-sprouter does not use a pump, so operation is cheaper and simpler than operation of the A-sprouter. Using an S-sprouter may be a more reasonable approach for removal of silver from solution or production of silver nanoparticles than using an A-sprouter.

Previous studies by Gardea-Torresdey et al. $(1998,2002,2003)$ have determined that alfalfa sprouts can also uptake $\mathrm{Au}^{\circ}$ and $\mathrm{Ag}^{\circ}$ from solid media to form nanoparticles. The solid medium was prepared using silver or gold solution as the source of metal. The literature indicates that the metal ions were reduced in the solid media before uptake by the sprouts. In the present study, we confirmed that the ions are indeed reduced by the media. Medium was prepared using only T.C. agar (Difco), distilled/deionized water, and either $\mathrm{AgNO}_{3}$ or $\mathrm{KAuCl}_{4}$ solution. This formulation differs slightly from the medium used by Gardea-Torresdey et al. (1998, 2002, 2003), which contains agar-agar instead of agar. Nonetheless, we observed that, over time, as the medium cooled to room temperature, colors characteristic of silver and gold nanoparticles gradually developed. The medium made with silver solution turned dark brown, and the color that evolved in the medium containing gold solution was pinkish-purple. For each case, the increase in color intensity continued for several hours until a final state was reached, beyond which the color remains unchanged. It is well known that silver and gold exhibit these colors only when present in nanoparticle form. 


\subsection{Extracellular Formation of Nanoparticles Using Pelargonium graveolens Extract}

\subsubsection{Formation of Silver Nanoparticles}

The reaction of geranium extract and $0.001 \mathrm{M} \mathrm{AgNO}_{3}$ was observed. For the procedure, three cultivars of geranium were used: 'Rose' (R), 'Rober's Lemon Rose' (RR), and 'Lady Plymouth' (LP). For each cultivar, four environmental conditions were examined for the reaction, and the jars were marked as follows:

1. exposure to light and excess oxygen (with lid removed)

2. exposure to light in limited oxygen (with lid on)

3. absence of light but excess oxygen (with lid removed)

4. absence of light and limited oxygen (with lid on)

Obvious color changes were noted as the reactions proceeded. The color that developed during these reactions is known to be characteristic of silver nanoparticles in solution. To record this visible change, all samples were photographed over the period of time during which the reaction occurred. Various observations were made, and preliminary conclusions regarding the nature of the reaction may be drawn based on this information.

Throughout this discussion describing the observations made during the reactions using geranium extract to form nanoparticles, it may be helpful to first define a few terms that will be used to make the explanation more clear. The time at which the reactants were combined will be defined as $\mathrm{t}=0$. Directly following this step, at $\mathrm{t}=\varepsilon$, some reaction mixtures were placed in darkness. The period of time in which the mixtures remain in darkness can be termed $t_{d}$. Because the amount of time that lapsed between 
mixing reactants and placing mixtures in the dark is so small, we can assume that $\varepsilon \approx 0$. These samples were eventually exposed to light for a period $t_{l}$, which takes place at $t>t_{d}$. Other samples had continuous exposure to light, and in this situation, $t_{d}=0$, and $t_{1} \neq 0$.

The first aspect of the reaction that became apparent was that the change in color, presumably due to formation of silver nanoparticles, takes place only in lighted conditions. This is clearly seen for each type of geranium reacting with $\mathrm{AgNO}_{3}$, but not when reacting with $\mathrm{KAuCl}_{4}$. Comparing images (a) and (b), (c) and (d), (e) and (f), and (g) and (h) from Figure 5.9 provides further support of this theory. The labels on the 2 jars in (a) are R1 and R2. Figures (a) through (d) show R and RR samples 5 hours after the reactants were combined, and figures (e) through (h) display the same samples 24 hours after combining reactants. All jars marked 1 and 2 (R1, RR1, LP1a, R2, RR2, and LP2a) were placed in light immediately upon combining reactants $\left(t_{d}=0\right)$. Jars marked 3 and 4 (R3, RR3, LP3a, R4, RR4, and LP4a) remained in the dark for a period $t_{d}$ (besides brief removal for taking pictures), but these jars would also eventually be subjected to light (see later discussion of Figure 5.10 on pp.44-45). Also see (i) from Figure 5.9 for images of LP (taken at 26 hours) demonstrating the hindrance of the reaction caused by lack of light. Although the labels for jars in (i) include an "a," the procedure was exactly the same as for reactions of $\mathrm{AgNO}_{3}$ with $\mathrm{R}$ and $\mathrm{RR}$.

Regarding the exposure to oxygen, no significant or obvious effect could be identified. This is made evident from examination of images in Figure 5.9 showing jars 1 and 2, which are under equal constraints except jar 1 is open to air and jar 2 is not. Likewise, jars marked 3 and 4 are exactly alike except for contact with oxygen. These figures show that, based on color, reactions are identical regardless of the degree of 
presence of oxygen. It must be understood that the reactions considered to occur in limited oxygen still had approximately $12 \%(\mathrm{v} / \mathrm{v})$ air present in the tightly closed jar. Therefore, although it appears that oxygen has no effect on the reaction, we cannot be certain that oxygen is not a reactant in the process taking place.

It was also found that after a length of time in darkness, the reaction would still begin upon exposure to light. Although postponed significantly, when the reaction was initiated, it appeared to take place as if it had not been delayed. Specifically, jars marked 1 and 2 (R1, RR1, LP1a, R2, RR2, and LP2a) were placed in light immediately upon combining reactants $\left(t_{d}=0\right)$. Jars marked 3 and $4(R 3, R R 3, L P 3 a, R 4, R R 4$, and LP4a) remained in the dark for a period $t_{d} \neq 0\left(t_{d}=13\right.$ days for $L P$ and $t_{d}=8$ weeks for $R$ and $\mathrm{RR}$ ) before they were exposed to light for a period $\mathrm{t}_{\mathrm{t}}$, yet no major difference in reaction time or intensity could be seen. Figure 5.10 shows the progress of the reaction of LP with $\mathrm{AgNO}_{3}$ that began after 13 days in darkness in images (a) through (d), which are taken at $t_{1}=1,4,7$, and 26 hours, respectively. In (e), a side-by-side comparison of jars LP1a, LP2a, LP3a, and LP4a is shown. LP1a and LP2a began reaction immediately $\left(\mathrm{t}_{\mathrm{d}}=\right.$ 0 ), while jars LP3a and LP4a had delayed reaction $\left(t_{d}=13\right.$ days). This picture shows LP3a and LP4a at $t_{1}=27$ hours, after exposure to light initiated the reaction, and LP1a and LP2a are shown at $t_{1}=14$ days. All jars in this figure have been diluted 20X after the reaction so the color differences are more pronounced, since discrepancy in colors is best displayed by solutions that have been diluted. In addition, $R$ and $R R$ also underwent the same treatment, where the jars that had been in darkness (in this case $t_{d}=8$ weeks) eventually were exposed to light. Although the progress versus time is not shown, the final products (R3, R4, RR3, and RR4 had $t_{d}=8$ weeks and $t_{1}=24$ hours, and R1, R2, 
RR1, and RR2 had $t_{d}=0$ and $t_{1}=8$ weeks +24 hours) are shown in Figure 5.11, again with 20X dilution. As mentioned before, jars labeled 1 and 2 underwent reaction immediately while jars 3 and 4 were kept in darkness until $t>t_{d}\left(t_{d}=8\right.$ weeks), when they began reaction upon exposure to light. It can be seen from these images that the solutions that were kept in darkness for an extended time (had $t_{d}>0$ ) still reacted similarly to those that were immediately exposed to light $\left(t_{d}=0\right)$. However, the solutions that had begun reacting more recently appear to be slightly lighter compared to those that had started reacting long before.

Among the cultivars, only a slight difference in reaction was noted. Based on the color changes, it was observed that all reactions proceeded over a similar time period, and appeared to reach steady state at approximately $t_{1}=24$ hours. Images from Figure 5.12, all diluted 20X after initially reacting, best illustrate the difference between 'Rose', 'Rober's Lemon Rose', and 'Lady Plymouth'. Image (a) shows jars labeled R1, R2, R3, and R4, (b) shows RR1, RR2, RR3, and RR4, and (c) shows labels LP1a, LP2a, LP3a, and LP4a. As already mentioned, all jars in (a), (b), and (c) marked 3 and 4 had undergone delayed reaction after a period of time in darkness ranging from $t_{d}=13$ days to $t_{d}=8$ weeks. Jars designated 1 and 2 were immediately subjected to light $\left(t_{d}=0\right)$, and therefore, the reaction in jars marked 1 and 2 had ended long ago (12 days to 55 days earlier). The reaction taking place in jars 3 and 4 had only ended in the last few hours, and all solutions were then diluted at the same time, whether the reaction had just ended or had ended long ago. RR is noticeably more yellow in color, lacking the orange tint shown by R. LP also has an orange tint, but it appears to be a darker shade than that 
exhibited by R. All colors were similar to the accepted color characteristic of silver nanoparticles in solution, which has been described as a yellow-brown color.



Figure 5.9. Reactions of $R, R R$, and LP extracts with $\mathrm{AgNO}_{3}$ pictured at (a) $t_{d}=0, t_{1}=5$ $\mathrm{hr}$, (b) $\mathrm{t}_{\mathrm{d}}=5 \mathrm{hr}, \mathrm{t}_{1}=0$, (c) $\mathrm{t}_{\mathrm{d}}=0, \mathrm{t}_{1}=5 \mathrm{hr}$, (d) $\mathrm{t}_{\mathrm{d}}=5 \mathrm{hr}, \mathrm{t}_{\mathrm{l}}=0$, (e) $\mathrm{t}_{\mathrm{d}}=0, \mathrm{t}_{1}=24 \mathrm{hr}$, (f) $\mathrm{t}_{\mathrm{d}}=$ $24 \mathrm{hr}, \mathrm{t}_{\mathrm{l}}=0,(\mathrm{~g}) \mathrm{t}_{\mathrm{d}}=0, \mathrm{t}_{\mathrm{l}}=24 \mathrm{hr}$, (h) $\mathrm{t}_{\mathrm{d}}=24 \mathrm{hr}, \mathrm{t}_{\mathrm{l}}=0$, and at (i) $\mathrm{td}=0, \mathrm{tl}=26 \mathrm{hr}$ for the two jars on the left, and $\mathrm{td}=26 \mathrm{hr}, \mathrm{tl}=0$ for the two jars on the right. 


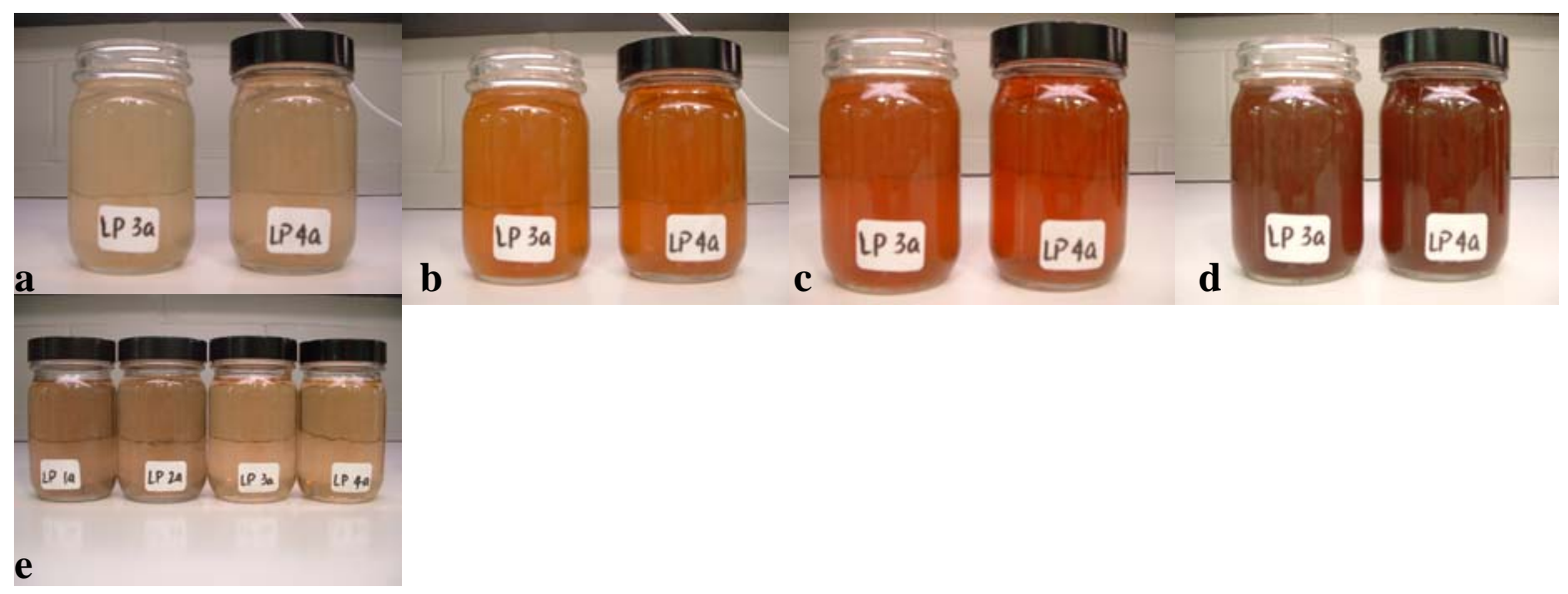

Figure 5.10. Reaction of LP extract with $\mathrm{AgNO}_{3}, \mathrm{t}_{\mathrm{d}}=13$ days, shown at (a) $\mathrm{t}_{1}=1 \mathrm{hr}$, (b) $t_{1}=4 \mathrm{hr}$, (c) $t_{1}=7 \mathrm{hr}$, and (d) $t_{1}=26 \mathrm{hr}$, and (e) final products after $20 \mathrm{X}$ dilution, with the two jars on the left having $t_{d}=0, t_{1}=14$ days and the two jars on the right having $t_{d}=13$ days, $\mathrm{t}_{1}=27 \mathrm{hr}$.

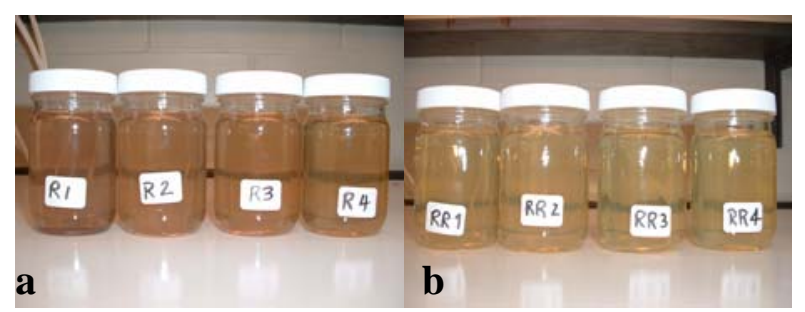

Figure 5.11. Reactions of $\mathrm{R}$ and $\mathrm{RR}$ extracts with $\mathrm{AgNO}_{3}$ after $20 \mathrm{X}$ dilution. For both (a) and (b), the two jars on the left have $t_{d}=0, t_{1}=8$ weeks $+24 \mathrm{hr}$, and the two jars on the right have $t_{d}=8$ weeks, $t_{l}=24 \mathrm{hr}$.

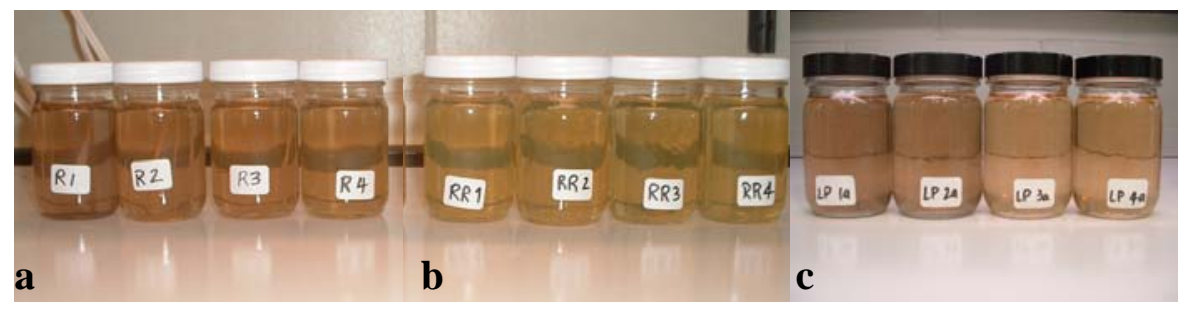

Figure 5.12. Final products from reactions of $\mathrm{R}, \mathrm{RR}$, and $\mathrm{LP}$ extracts with $\mathrm{AgNO}_{3}$ after $20 X$ dilution. For each of (a), (b), and (c), the two jars on the left have $t_{d}=0$, and the two jars on the right have $t_{d} \neq 0$. 
Samples were later analyzed using a spectrophotometer to observe spectra generated over visible wavelengths ranging from 400-700 nm. Although this analysis was not conducted until much time had passed (approximately 3 months), all samples remained homogeneous in appearance and free of contamination and appeared to be stable. This is probably due to the well-known antibacterial property of silver nanoparticles, which have been incorporated into a multitude of products that exploit this trait. Table 5.1 shows positions of peaks for samples representing each cultivar, as well as comparable data from the literature (Shankar et al. 2003b). While the spectra from R and RR demonstrate similar characteristics, the data from LP differs from these two. The peak position for LP is not much shifted, but a broad shoulder is distinctly present at the region around $550 \mathrm{~nm}$, maybe indicating the presence of more anisotropic particles relative to reactions with $R$ and $R R$.

Table 5.1. Peak wavelengths from spectra of silver nanoparticles produced by the reaction of extract from various cultivars of $P$. graveolens with $0.001 \mathrm{M} \mathrm{AgNO}_{3}$

\begin{tabular}{|l|l|}
\hline Sample & Wavelength of Peak (nm) \\
\hline R & 421 \\
\hline RR & 410 \\
\hline LP & 413 \\
\hline $\begin{array}{l}\text { unknown cultivar } \\
\text { (Shankar et al. 2003b) }\end{array}$ & 440 \\
\hline
\end{tabular}

It is known that many reactions are sensitive to $\mathrm{pH}$ changes. Thus, the influence of $\mathrm{pH}$ on the reaction involving geranium extract and silver ions was investigated. The 
$\mathrm{pH}$ values were measured periodically to study the evolution of $\mathrm{pH}$ as the reactions proceed, and the results are represented in Figures 5.13 and 5.14. These figures show the change in $\mathrm{pH}$ over time for the reactions involving reduction of $\mathrm{Ag}^{+}$in $0.001 \mathrm{M} \mathrm{AgNO}_{3}$ solution. The trends observed for both R and LP were similar to each other, but the $\mathrm{pH}$ values for the reaction of R extract are slightly lower than for the reaction of LP extract. In each case, however, we see that the $\mathrm{pH}$ initially increases, but then starts to drop somewhere between 30 and 60 minutes and becomes stable after 1.5 hours. The overall change in $\mathrm{pH}$ values over the course of the reaction is really quite small, with no two values differing more than $0.4 \mathrm{pH}$ units.

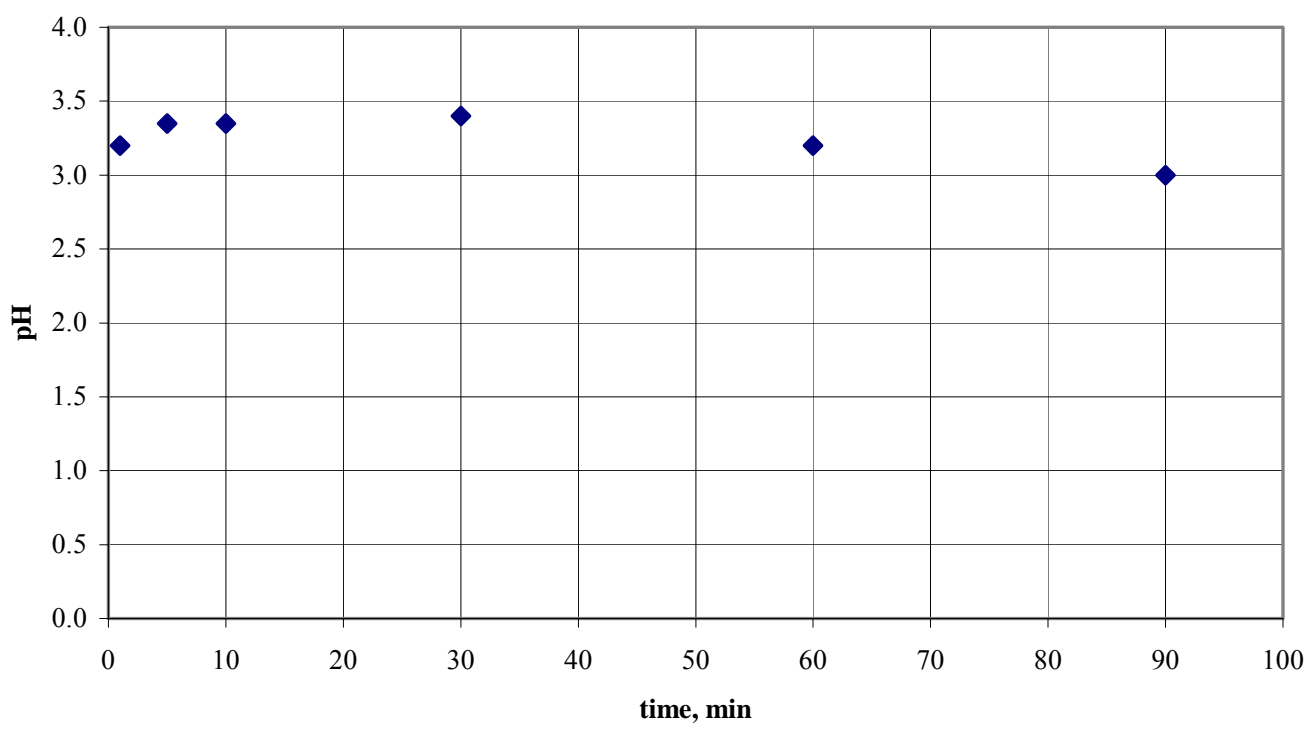

Figure 5.13. Progression of $\mathrm{pH}$ over time upon reaction of $10 \mathrm{ml} \mathrm{R}$ extract with $100 \mathrm{ml} 0.001 \mathrm{M} \mathrm{AgNO}_{3}$ solution. 


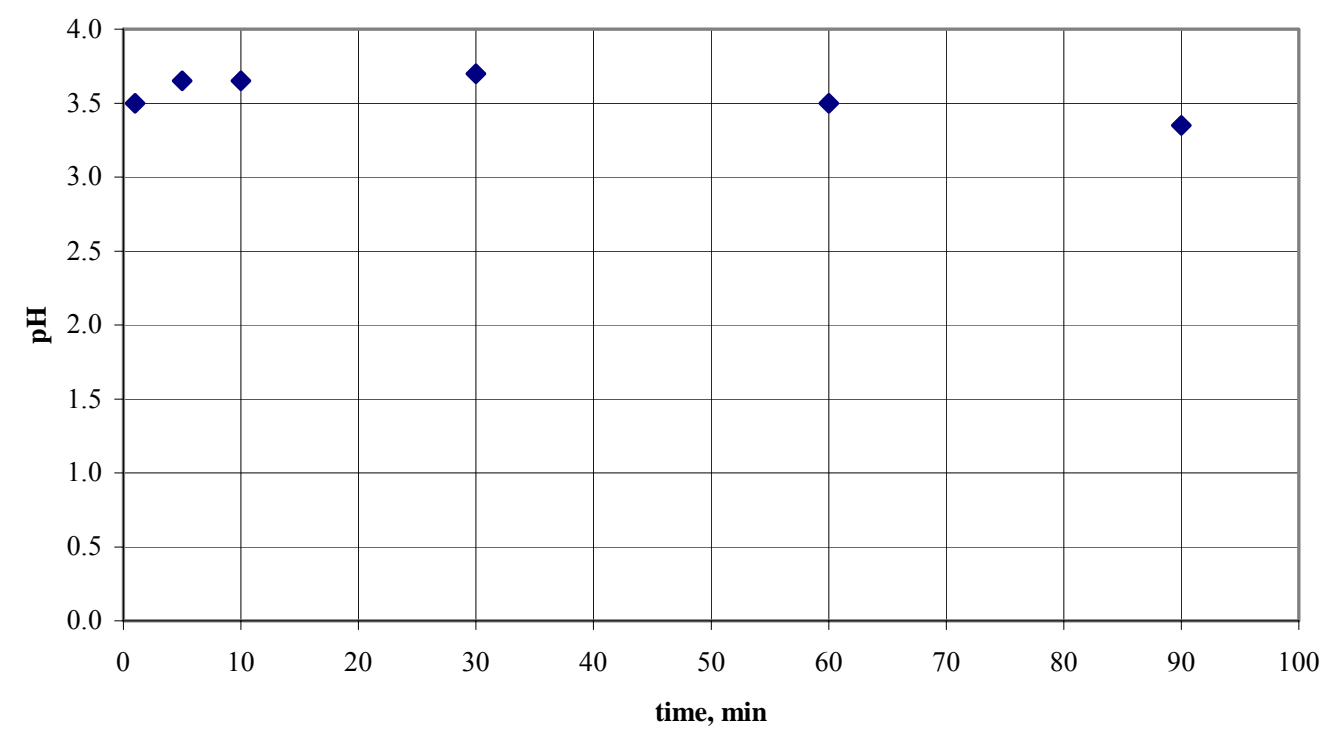

Figure 5.14. Progression of $\mathrm{pH}$ over time upon reaction of $10 \mathrm{ml} \mathrm{LP}$ extract with $100 \mathrm{ml} 0.001 \mathrm{M} \mathrm{AgNO}_{3}$ solution.

\subsubsection{Formation of Gold Nanoparticles}

A parallel experiment was carried out using $0.001 \mathrm{M} \mathrm{KAuCl}_{4}$. Again, all three varieties of geranium were used and equivalent conditions were tested. A color change was once again detected, analogous to that of $\mathrm{AgNO}_{3}$ experiments that had evolved over time. However, this color transformation was much more abrupt, and the time needed to reach its final state was much shorter. This reaction produced a final hue that was pinkish, which is consistent with the literature (Shankar et al. 2003a, 2004a), or blue as opposed to yellow-brown. It should be noted that at the beginning of each reaction, a yellow-orange color was initially observed (see (a), (b), and (e) in Figure 5.15), but the reaction progressed quickly and the mixture became very dark, indicating a high concentration of nanoparticles. Images (a) and (b) show RR samples at $t=20 \mathrm{~min}$, and (c) depicts the same samples after 65 minutes of reaction. Likewise, (e) shows LP 
samples after 5 min of reaction, and (f) shows the same samples after the reaction had progressed for $30 \mathrm{~min}$. By this time, the colors could not easily be discerned, but after 20X dilution it could be seen that the colors of each reaction mixture of geranium and $\mathrm{KAuCl}_{4}$ had changed drastically from yellow-orange to the pinkish hue ( $\mathrm{R}$ and $\mathrm{RR}$ ) or blue color (LP) of the final product, seen in images (d) and (g). The labels in (d) are $\mathrm{RR} 1 \mathrm{~b}, \mathrm{RR} 2 \mathrm{~b}, \mathrm{RR} 3 \mathrm{~b}$, and $\mathrm{RR} 4 \mathrm{~b}$, , $\mathrm{b}$ ” indicating reaction with $\mathrm{KAuCl}_{4}$.

In contrast to previous experiments with $\mathrm{AgNO}_{3}$, this reaction was not remarkably influenced by light. For example, for LP, at first a slight inconsistency in color intensity could be seen as shown for $\mathrm{t}=30 \mathrm{~min}$ under Figure 5.16(a), where the two jars on the left have full exposure to light and the two jars on the right have been protected from light. Yet as the reaction proceeded, the difference became imperceptible. Samples observed only 10 min later, as shown in (b), demonstrate the basis for this assertion. At the finish of the reaction (see (c), (d), and (e)), all specimens, after 20X dilution, looked identical, despite involvement of light. From left to right, the labels in (d) are R1b, R2b, R3b, R4b, and labels in (e) are RR1b, RR2b, RR3b, and RR4b. Reactions involving R and RR had no obvious difference between jars that were exposed to light and those shielded from light.

Oxygen, as well, did not cause a significant visible impact on the progression of the reaction. For RR, (b) and (e) from Figure 5.17 prove that both early in the reaction and after ample time has passed, oxygen has no major effect. Image (b) shows the reaction after 12 minutes, and (e) shows $R R 1 b, R R 2 b, R R 3 b$, and $R R 4 b$ after the reaction ended and samples were diluted 20X. Jars marked 1 and 3 were allowed to react in the presence of excess oxygen, but jars marked 2 and 4 were exposed to limited oxygen only. 
LP also appears not to be greatly affected by oxygen, as seen in (f), (g), and (h), which respectively show the progress of the reaction at 5 minutes, 30 minutes, and samples diluted 20X at 24 hours. The effect of oxygen with R, however, is not as well-defined. Figure 5.17(a) compares images of two samples of R, one exposed to excess oxygen, and the other, not. It appears that the reaction is indeed altered by the presence of oxygen. Despite this observation, it must be understood that while effort was made to ensure that reactions began at nearly the same time, some limitations are unavoidable, and the reaction involving sample $\mathrm{R} 2 \mathrm{~b}$ was slightly delayed. This may account for the difference in color, which, as seen in (c) for the same two bottles after about 15 minutes of reaction, is unnoticeable. Figure (d) provides verification that all samples of $\mathrm{R}$ at $\mathrm{t}=$ infinity, whether exposed to air or not, have identical end results.

Finally, differences among cultivars must be considered. All reactions began very quickly (colors were obvious after only 5 minutes) and lasted only about 1 hour or slightly less as indicated from visible change in intensity of color. At 15 minutes, it can be seen in (a) from Figure 5.18 that $\mathrm{R}$ reacted much more rapidly than RR, since both reactions were initiated at the same time. The following image (b) (showing $t=60 \mathrm{~min}$ ) shows that the difference became much smaller after time passed from 15 to 60 minutes.

However, the final results revealed a noticeable difference in color between plant varieties (see Figure 5.19). 'Lady Plymouth' especially stood apart from the others, developing a deep blue color, whereas both 'Rose' and 'Rober's Lemon Rose' reacted to produce a pinkish red tint. RR, shown in image (a), is the lightest shade of the three, just as it had been with regard to the $\mathrm{AgNO}_{3}$ reaction. Also in this investigation, $\mathrm{RR}$ (shown in(a)) and R (see (b)) again produced very similar results, while LP (seen in (c)) proved 
quite different. The final color produced by $\mathrm{R}$ and $\mathrm{RR}$ turned out as anticipated. It has been reported many times that spherical gold nanoparticles exhibit a ruby-red color in solution. LP, on the other hand, produced a color that was quite unexpected. Since gold nanoparticles can have a vast array of shapes and sizes, these findings suggested that this particular plant is forming anisotropic gold nanoparticles. 


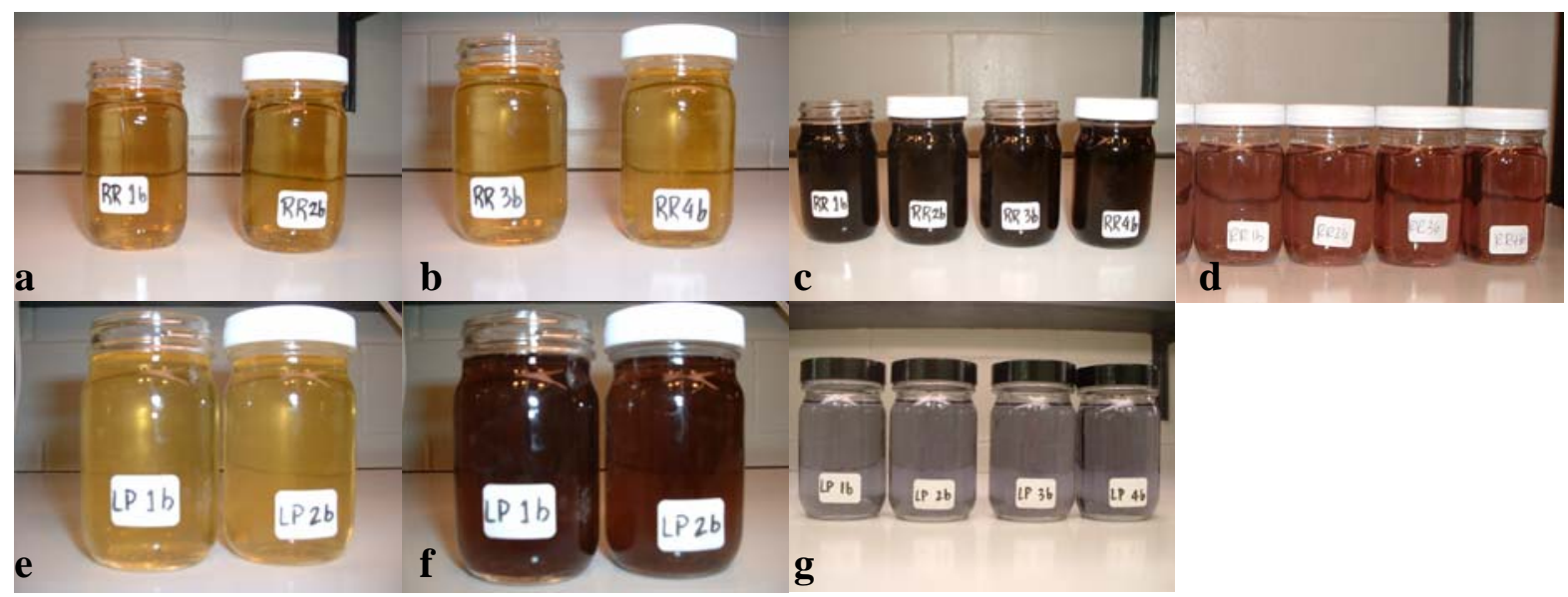

Figure 5.15. Reactions of RR and LP extracts with $\mathrm{KAuCl}_{4}$ showing (a) $t_{d}=0, t_{1}=20$ $\min (\mathrm{b}) t_{d}=20 \min , t_{1}=0$ (c) $t_{d}=0, t_{1}=65$ min for the two jars on the left, and $t_{d}=65$ min, $t_{1}=0$ for the two jars on the right (d) final RR products diluted $20 X$, (e) $t_{d}=0, t_{1}=5$ $\min (f) t_{d}=0, t_{1}=30 \mathrm{~min}$, and (g) final LP products diluted $20 \mathrm{X}$.

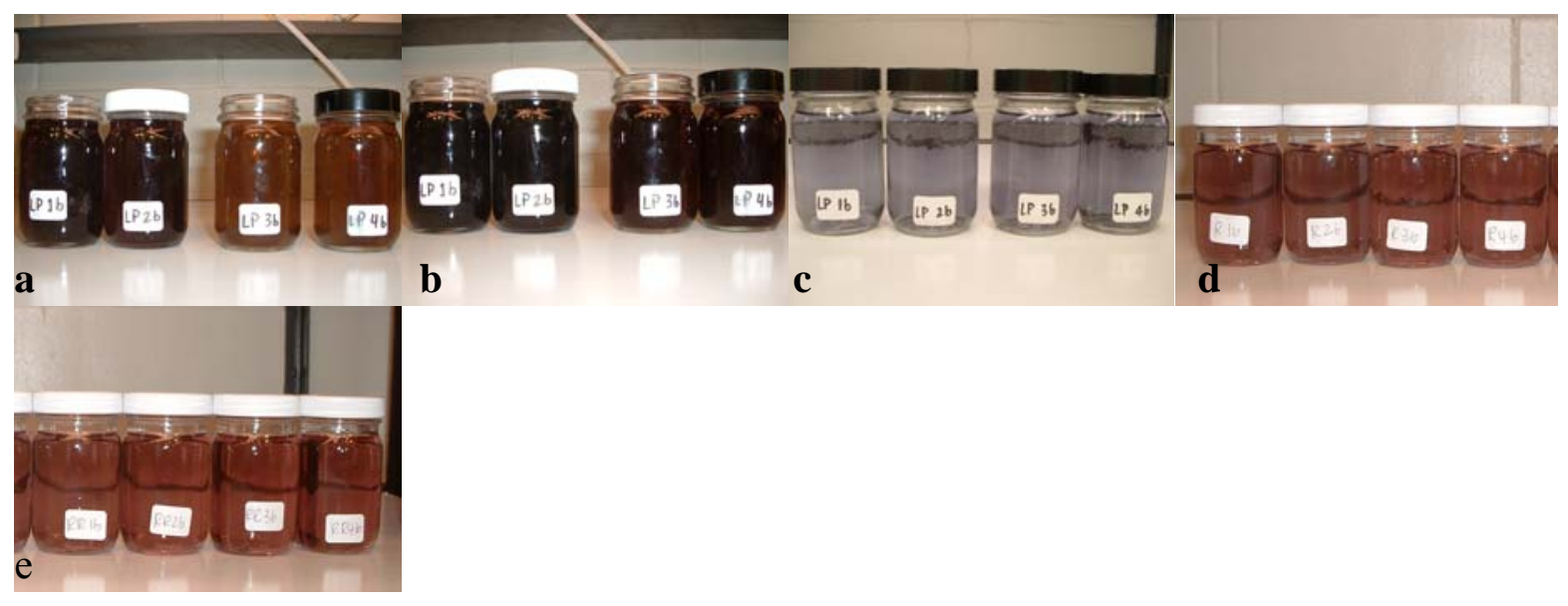

Figure 5.16. Reactions of $\mathrm{LP}, \mathrm{R}$, and $\mathrm{RR}$ extracts with $\mathrm{KAuCl}_{4}$ showing (a) $\mathrm{t}=30 \mathrm{~min}$, (b) $\mathrm{t}=40 \mathrm{~min}$, (c) final LP products diluted 20X, (d) final $\mathrm{R}$ products diluted 20X, and (e) final RR products diluted 20X. All jars labeled 1 and 2 reacted in lighted conditions, and jars 3 and 4 all underwent reaction in darkness. 


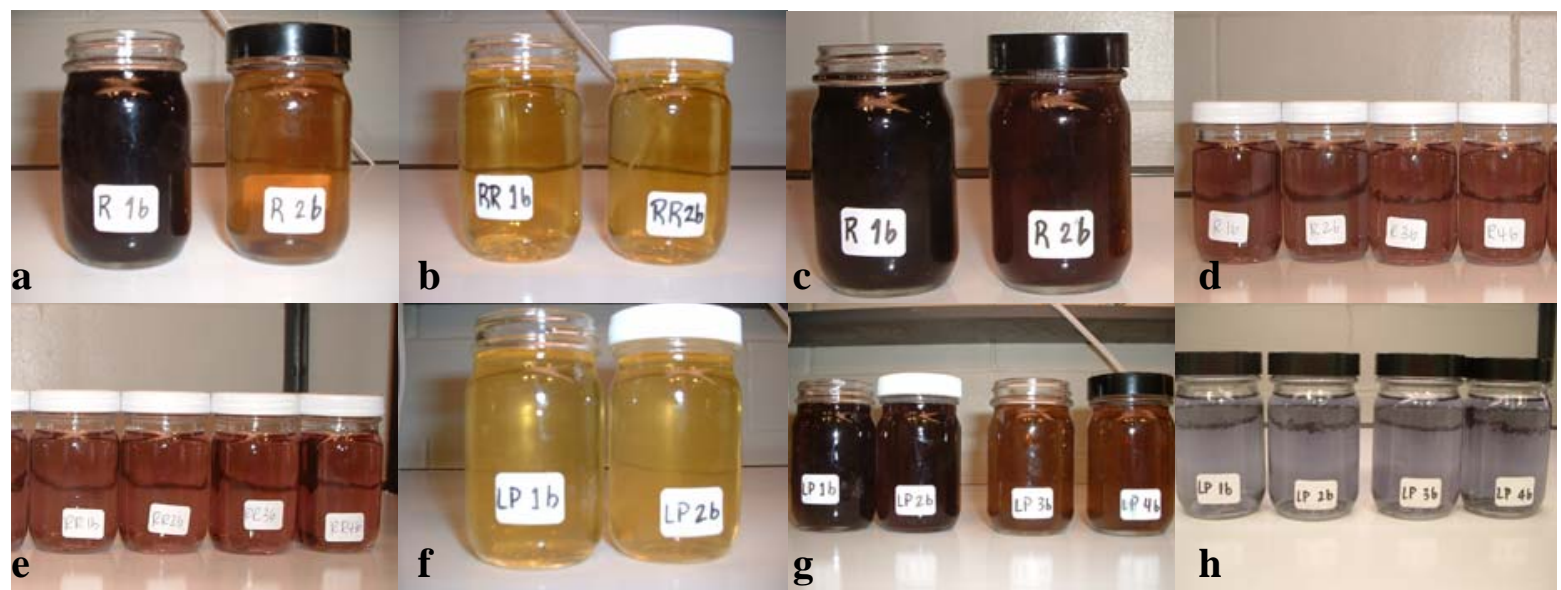

Figure 5.17. Reactions of $\mathrm{R}, \mathrm{RR}$, and $\mathrm{LP}$ extracts with $\mathrm{KAuCl}_{4}$ showing (a) $\mathrm{t}=10 \mathrm{~min}$, (b) $\mathrm{t}=12 \mathrm{~min}$, (c) $\mathrm{t}=15 \mathrm{~min}$, (d) final $\mathrm{R}$ products diluted $20 \mathrm{X}$, (e) (f) $\mathrm{t}=5 \mathrm{~min},(\mathrm{~g}) \mathrm{t}=30$ min, (h) final LP products diluted 20X. All jars labeled 1 and 2 reacted in lighted conditions, and jars 3 and 4 all underwent reaction in darkness. Jars 1 and 3 were exposed to excess oxygen, while jars 2 and 4 reacted in a limited oxygen environment.

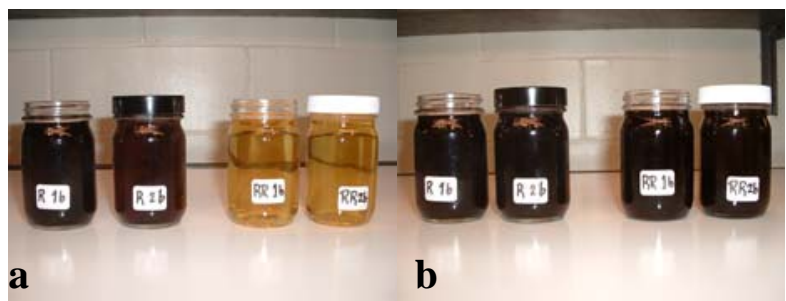

Figure 5.18. Reactions of $\mathrm{R}$ and $\mathrm{RR}$ extracts with $\mathrm{KAuCl}_{4}$ showing (a) $t=15 \mathrm{~min}$, and (b) $\mathrm{t}=60 \mathrm{~min}$.



Figure 5.19. Reactions of $\mathrm{RR}, \mathrm{R}$, and $\mathrm{LP}$ extracts with $\mathrm{KAuCl}_{4}$ showing final products diluted 20X for (a) RR, (b) R, and (c) LP.

Further investigation of these reactions was accomplished by analyzing the visible light spectra (400 nm-700 nm) using a Spectronic Genesys 5 Spectrophotometer. Spectra 
analyses were not performed until several months after the reactions were initiated. However, most solutions appeared to be stable after this length of time, save a few that had become contaminated. Samples R1b, RR1b, and LP1b were free of visible contamination and thus were chosen for analysis.

Spectra were compared with the results attained by Shankar et al. (2003a). The following table displays the wavelength for peaks processed for each cultivar as well as that indicated in the study by Shankar et al. (2003a). For both R and RR, peaks were present at wavelengths relatively close to those reported by Shankar et al (2003a) for reaction of geranium extract with $0.001 \mathrm{M} \mathrm{KAuCl}_{4}$ solution. LP yielded spectra that were much different from those of $\mathrm{R}$ and RR, and that generated by Shankar (2003a). Its spectrum exhibits two distinct peaks, possibly corresponding to excitation of the transverse and longitudinal plasmon vibrations, again suggesting formation of anisotropic nanostructures. One of these peaks occurs at a wavelength reasonably close to the rest, but the other peak appears at a much higher wavelength.

Table 5.2. Peak wavelengths from spectra of gold nanoparticles produced by the reaction of extract from various cultivars of $P$. graveolens with $0.001 \mathrm{M} \mathrm{KAuCl}_{4}$

\begin{tabular}{|l|l|}
\hline Sample & Wavelength of Peaks (nm) \\
\hline R & 526 \\
\hline RR & 526 \\
\hline LP & 540,646 \\
\hline $\begin{array}{l}\text { unknown cultivar } \\
\text { (Shankar et al. 2003b) }\end{array}$ & 551 \\
\hline
\end{tabular}


Figure 5.20 shows spectra of two different solutions containing gold nanoparticles. In (a), the nanoparticles were formed by the reduction of $\mathrm{Au}^{3+}$ using extract of the 'Rose' (R) cultivar of scented geranium. The spectra of a solution that has undergone an equivalent reaction with 'Lady Plymouth' (LP) is shown in (b). These two spectra further demonstrate the cultivar dependency of the reaction, since the curves are quite different from one another. A single peak is displayed in (a), whereas two distinct peaks are evident in (b). As explained previously, the occurrence of two peaks in the spectrum in (b) is believed to indicate the presence of anisotropic nanoparticles, and the two peaks probably correspond to the longitudinal and transverse plasmon resonances exhibited by the particles. On the other hand, the single peak shown in (a) likely indicates that the particles are mostly spherical, or have an aspect ratio very close to 1 . Though it is not shown, the spectrum for 'Rober's Lemon Rose' is nearly identical to that for 'Rose'. It is the spectrum for 'Lady Plymouth' that stands apart from the other two, both for reactions with $\mathrm{KAuCl}_{4}$ and $\mathrm{AgNO}_{3}$. 
(a)

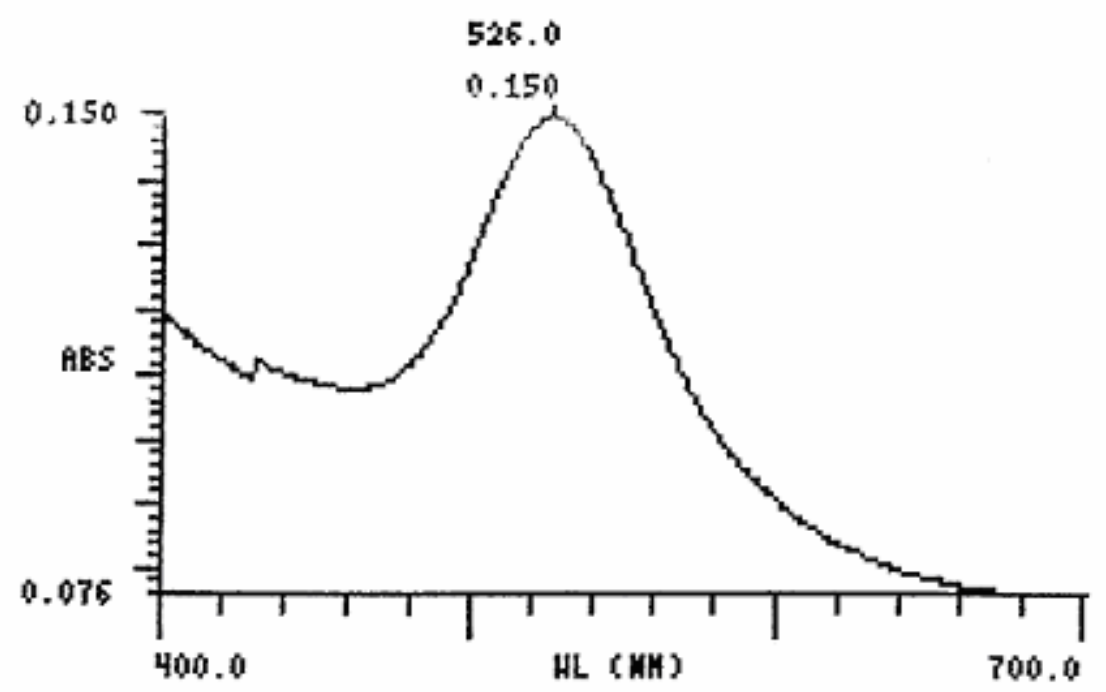

(b)

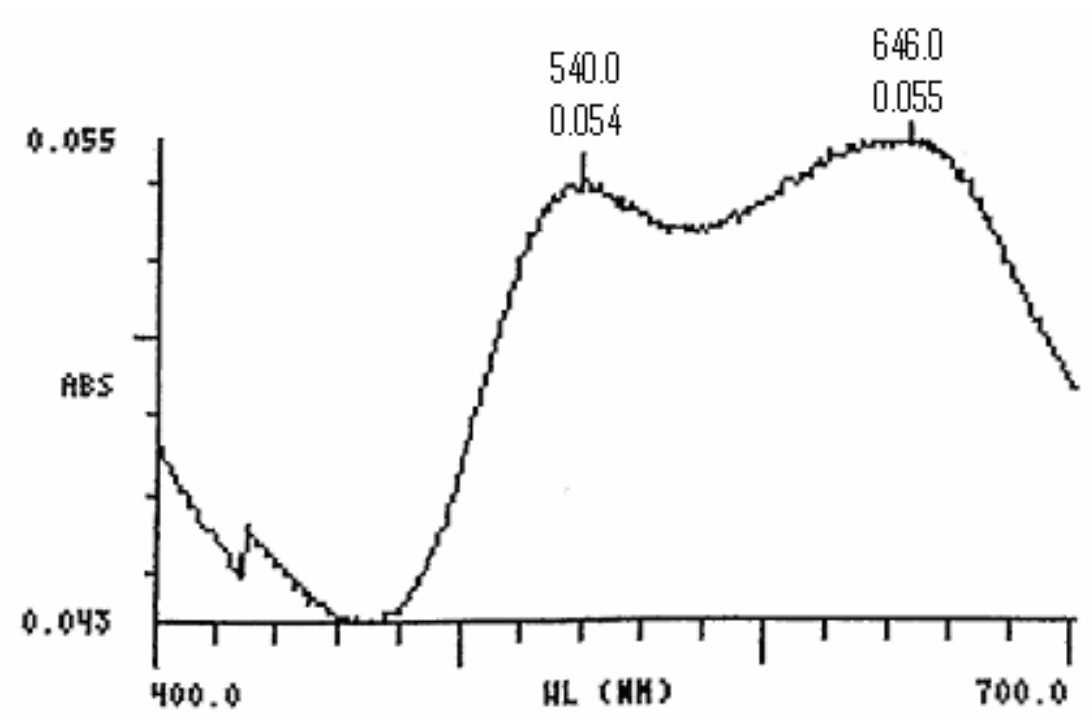

Figure 5.20. Absorbance spectra of samples of a solution containing gold nanoparticles (a) after reaction of $\mathrm{R}$ extract with $\mathrm{KAuCl}_{4}$, and (b) after reaction of $\mathrm{LP}$ extract with $\mathrm{KAuCl}_{4}$. 
As was mentioned in Section 5.5.1, $\mathrm{pH}$ can often significantly affect reactions, and $\mathrm{pH}$ measurements may provide insight into the mechanics of the reaction. Therefore, studies were conducted to observe fluctuations in $\mathrm{pH}$ as the reaction progressed. Figures 5.21 and 5.22 show that the $\mathrm{pH}$ gradually decreases as the reaction of either LP or R extract with $0.001 \mathrm{M} \mathrm{KAuCl}_{4}$ proceeds. This is dissimilar to the reaction for reduction of silver, in which the $\mathrm{pH}$ initially increases before decreasing and becoming stable. As seen before, the $\mathrm{pH}$ curve for the reaction of $\mathrm{R}$ extract is shifted slightly lower than the curve for the reaction of LP extract, but in both cases, the overall $\mathrm{pH}$ change is very small. It has been previously observed that this reaction reaches near completion after approximately one hour. These figures show that, for both $\mathrm{R}$ and LP, the $\mathrm{pH}$ levels off as the reaction nears its endpoint. The solutions were tested again after 15 hours and 41 hours, and it was found that the $\mathrm{pH}$ values of each solution had been maintained over these lengths of time. 


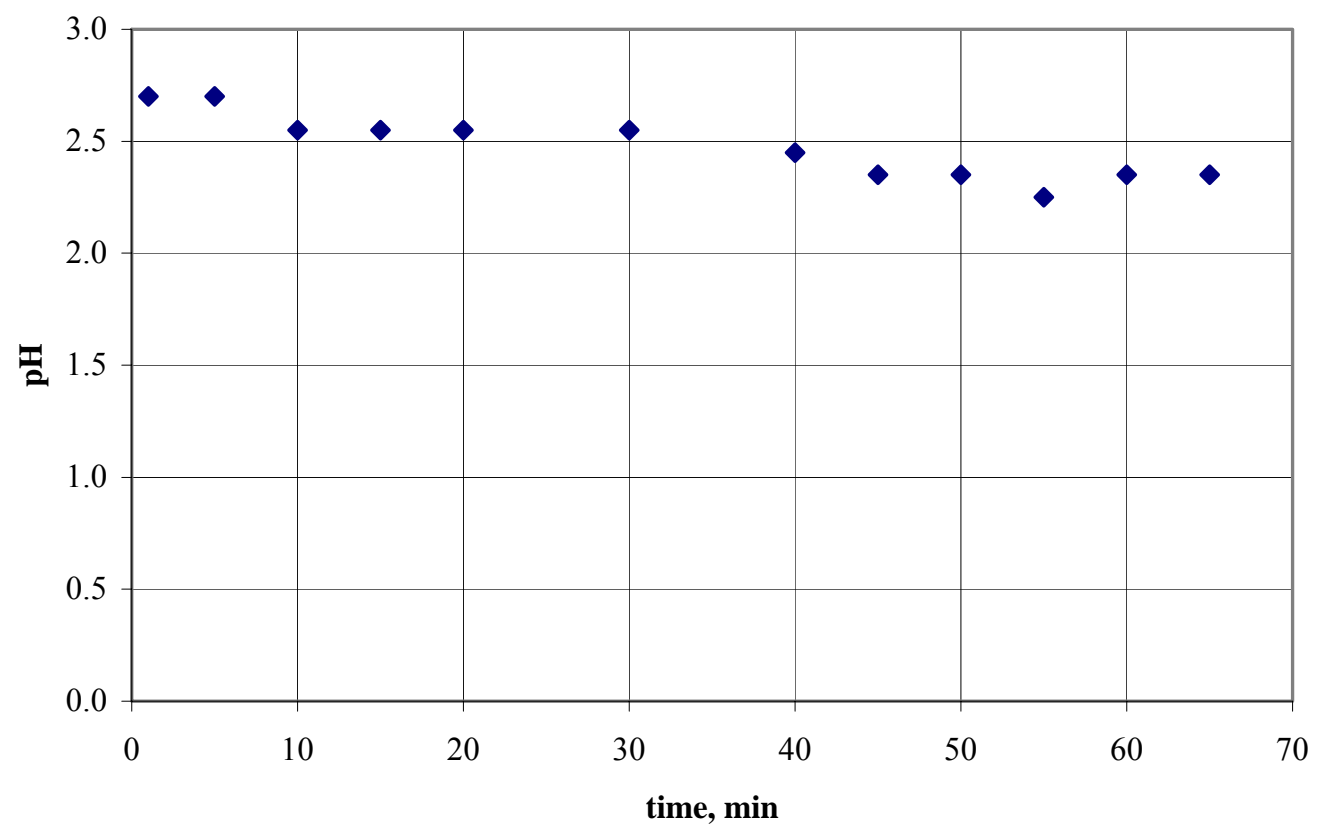

Figure 5.21. Progression of $\mathrm{pH}$ over time upon reaction of $10 \mathrm{ml} \mathrm{R}$ extract with $100 \mathrm{ml} 0.001 \mathrm{M} \mathrm{KAuCl}_{4}$ solution.

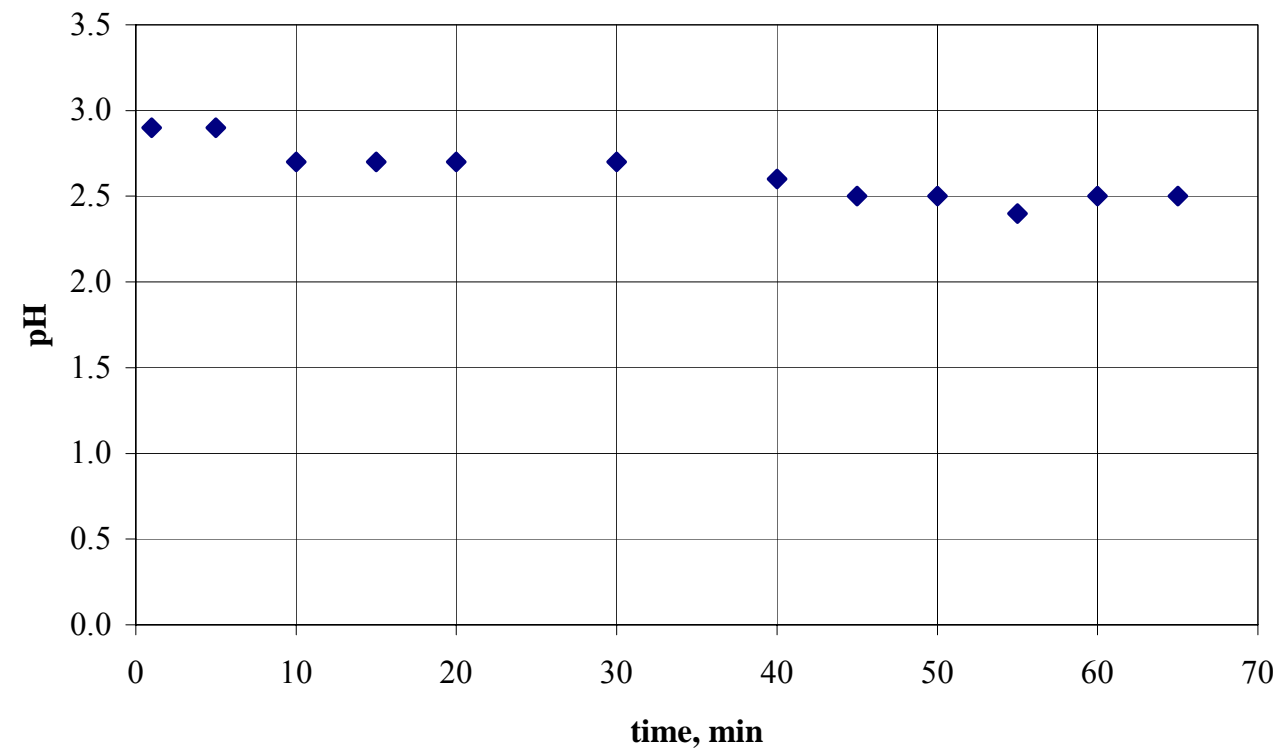

Figure 5.22. Progression of $\mathrm{pH}$ over time upon reaction of $10 \mathrm{ml} \mathrm{LP}$ extract with $100 \mathrm{ml} 0.001 \mathrm{M} \mathrm{KAuCl}_{4}$ solution. 
Furthermore, the process for synthesis of gold nanoparticles using LP was carried out under a controlled $\mathrm{pH}$ environment. Immediately following initiation of the reaction, the solution was adjusted to have a specific $\mathrm{pH}$ value. The reaction was observed for four LP samples with $\mathrm{pH}$ values of $3.5,4.0,4.5$, and 6.0. In an uncontrolled environment, the initial $\mathrm{pH}$ value for the reaction of $10 \mathrm{ml} \mathrm{LP}$ extract with $100 \mathrm{ml} 0.001 \mathrm{M} \mathrm{KAuCl}_{4}$ is 2.9 , as seen in Figure 5.22. (Figure 5.26 (d) shows the corresponding gold nanoparticle solution, labeled LP, which was produced in an uncontrolled $\mathrm{pH}$ environment with an initial $\mathrm{pH}$ value of 2.9. It should be noted that the color is similar to that seen for the controlled $\mathrm{pH}$ 3.5.) Thus, the conditions for the reactions with controlled $\mathrm{pH}$ values are not radically different from the natural reaction environment. Figure 5.23 shows the mixtures produced from these reactions. From the difference in colors, it is clear that the $\mathrm{pH}$ of the solution indeed affects the reaction. The products formed exhibit colors that change gradually in color, starting as dark blue and becoming more pinkish in shade as $\mathrm{pH}$ increases from 3.5 to 6.0. Spectrophotometry analysis was performed on these diluted samples to generate spectra of absorbance over wavelengths of visible light (400 $\mathrm{nm}-700 \mathrm{~nm}$ ). The spectra displayed single SPR bands that became narrower and increased in intensity as the $\mathrm{pH}$ increased. Also, the bands gradually shifted from $532 \mathrm{~nm}$ to $523 \mathrm{~nm}$ as the $\mathrm{pH}$ increased from 3.5 to 6.0 . 


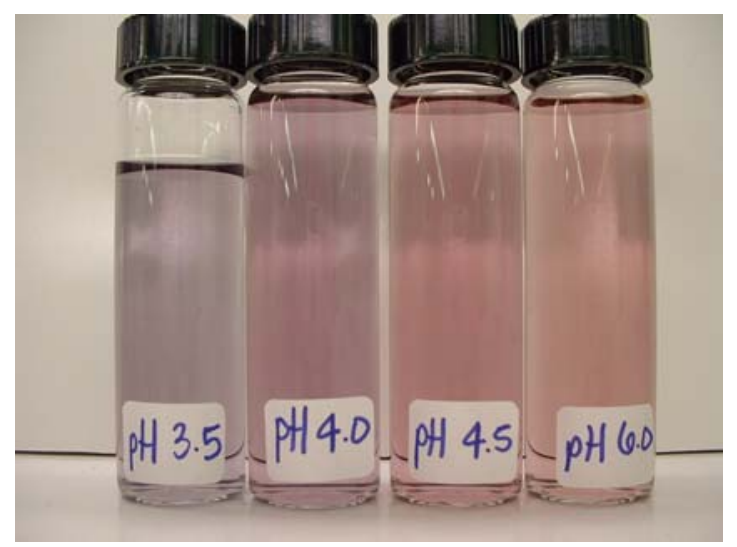

Figure 5.23. Products from reactions of $10 \mathrm{ml} \mathrm{LP}$ extract with $100 \mathrm{ml} 0.001$ $\mathrm{M} \mathrm{KAuCl}_{4}$ solution with initial reaction $\mathrm{pH}$ adjusted (from left to right) to 3.5, 4.0, 4.5, and 6.0. Samples shown were diluted 20X following reaction.

Following experiments were carried out with modifications to the procedure to enable a deeper understanding of factors affecting the reactions, besides exposure to light and oxygen. One such study examined the influence boiling time had on the reaction by reacting samples of geranium leaf extract that had been prepared with boiling times ranging from 0 minutes (no exposure to heat) to 20 minutes boiling. Further studies investigated the effect of boiling extract after leaves had been filtered out with Whatman \#1 filter paper (pore size of $11 \mu \mathrm{m}$ ). Therefore, most of the cell membranes would presumably be filtered out and could no longer influence the composition of the extract. However, it is reasonable to hypothesize that the extract composition is influenced by the length of time the leaves are submersed in the water before being filtered out. Thus, since this time period was not strictly regulated, it is suspected that the particular method used may produce inconsistent results, especially with respect to absorbance, which is dependent on the concentration of the sample. Although it cannot be stated with confidence, this complication may have less effect on the wavelength. 
The data gathered from these experiments are shown in Figures 5.24 and 5.25. Although notable differences exist, there appears to be no distinct relationship between absorbance and boiling time or wavelength and boiling time. However, it can be seen that for each test, the difference between boiling and not boiling is quite significant, since the wavelength drops sharply between boiling times of zero and one minute and the absorbance increases over this time interval.

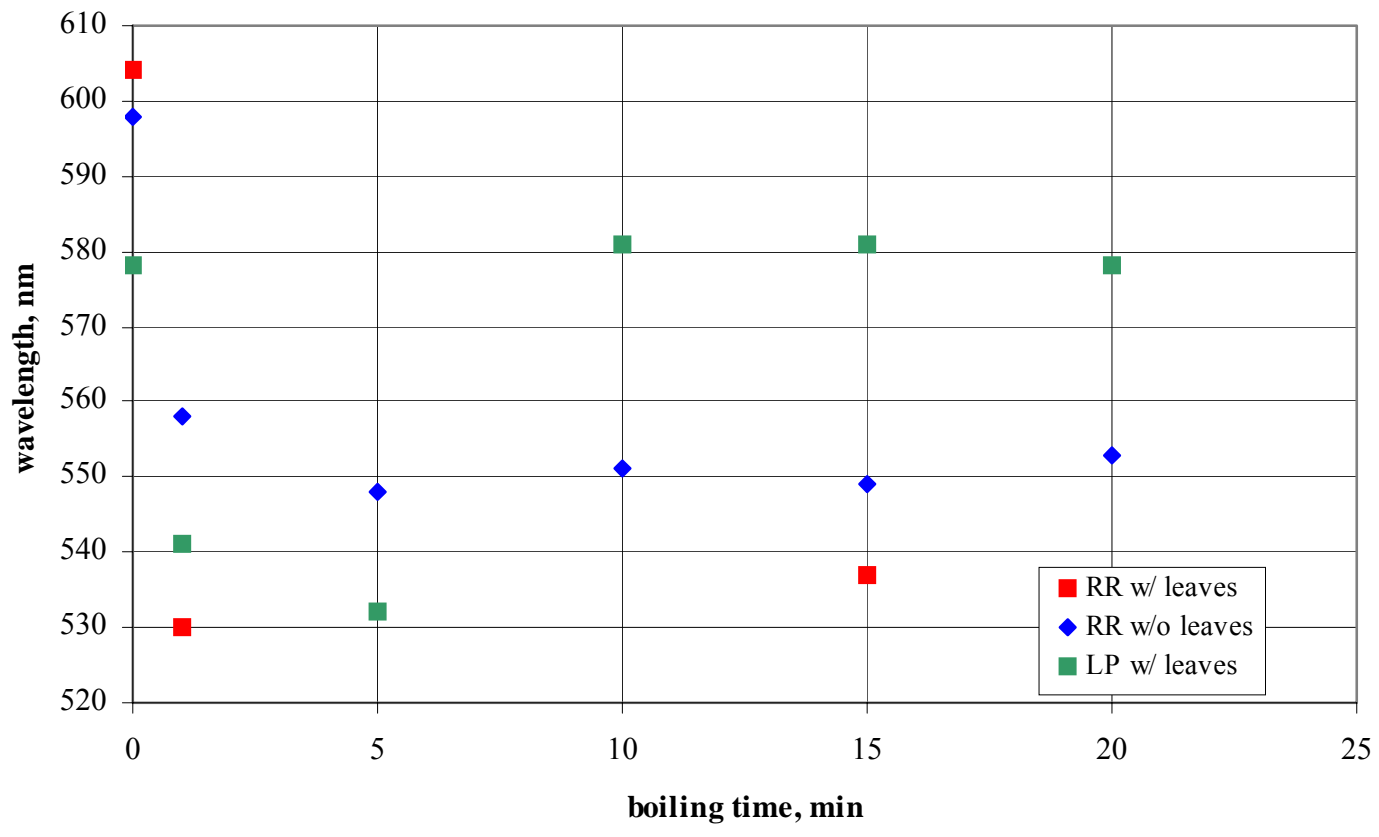

Figure 5.24. Peak wavelength from visible light spectra from reactions of geranium extract with $1 \times 10^{-4} \mathrm{M} \mathrm{AgNO}_{3}$ vs. extract boiling time. 


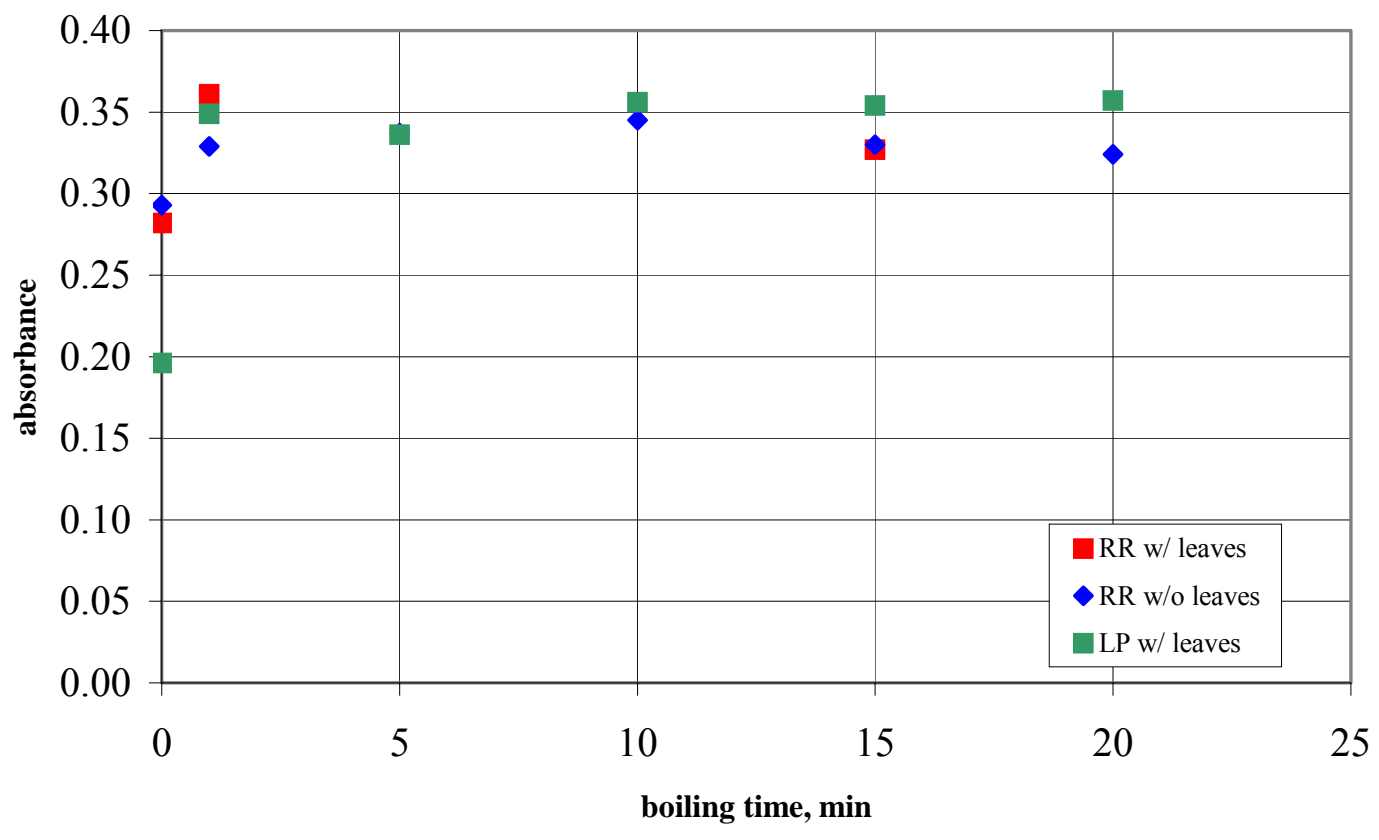

Figure 5.25. Peak absorbance from visible light spectra from reactions of geranium extract with $1 \times 10^{-4} \mathrm{M} \mathrm{AgNO}_{3}$ vs. extract boiling time.

\subsubsection{Comparison of Biological and Chemical Methods for Nanoparticle Synthesis}

The reduction of silver and gold ions and formation of nanoparticles using plant extracts has been reported many times (Ankamwar et al. 2005, Chandran et al. 2006, Rai et al. 2006, Shankar et al. 2003a, 2003b, 2004a, 2004b, 2004c, 2005). These nanoparticles have been characterized using several analytical techniques, including TEM and AFM imaging, XRD, and UV-vis-NIR spectrophotometry. To further support the assertion that formation of silver and gold nanoparticles occurs due to the reduction of metal ions with geranium extract, $\mathrm{NaBH}_{4}$ (sodium borohydride) was used to chemically simulate the reaction. $\mathrm{NaBH}_{4}$ is a widely used reducing agent, and its ability to reduce various metal ions, including silver and gold, is well known. Experiments were conducted using $\mathrm{NaBH}_{4}$ to reduce silver and gold ions in solutions of $0.001 \mathrm{M} \mathrm{AgNO}_{3}$ and $0.001 \mathrm{M} \mathrm{KAuCl}_{4}$, with no involvement of geranium extract. Prior to the reaction, the 
$\mathrm{AgNO}_{3}$ solution is clear and the $\mathrm{KAuCl}_{4}$ solution has a pale yellow color, but is also transparent. These solutions can be seen in (a) of Figure 5.26. Addition of small amounts of $\mathrm{NaBH}_{4}$ powder to the silver or gold solutions resulted in immediate reduction of ions, causing striking displays of color. The colors produced, shown in (b) of Figure 5.26, are typical of silver and gold nanoparticles in solution, and are characteristic only of particles with nanoscale dimensions. It is of key importance that similar colors were obtained by reduction of silver and gold ions using geranium extract. Image (c) enables the comparison of silver nanoparticles produced using different reducing agents $\left(\mathrm{NaBH}_{4}\right.$, LP extract, and R extract). Likewise, (d) shows solutions containing gold nanoparticles reduced by $\mathrm{NaBH}_{4}$ as well as $\mathrm{R}$ and $\mathrm{LP}$ extracts. 


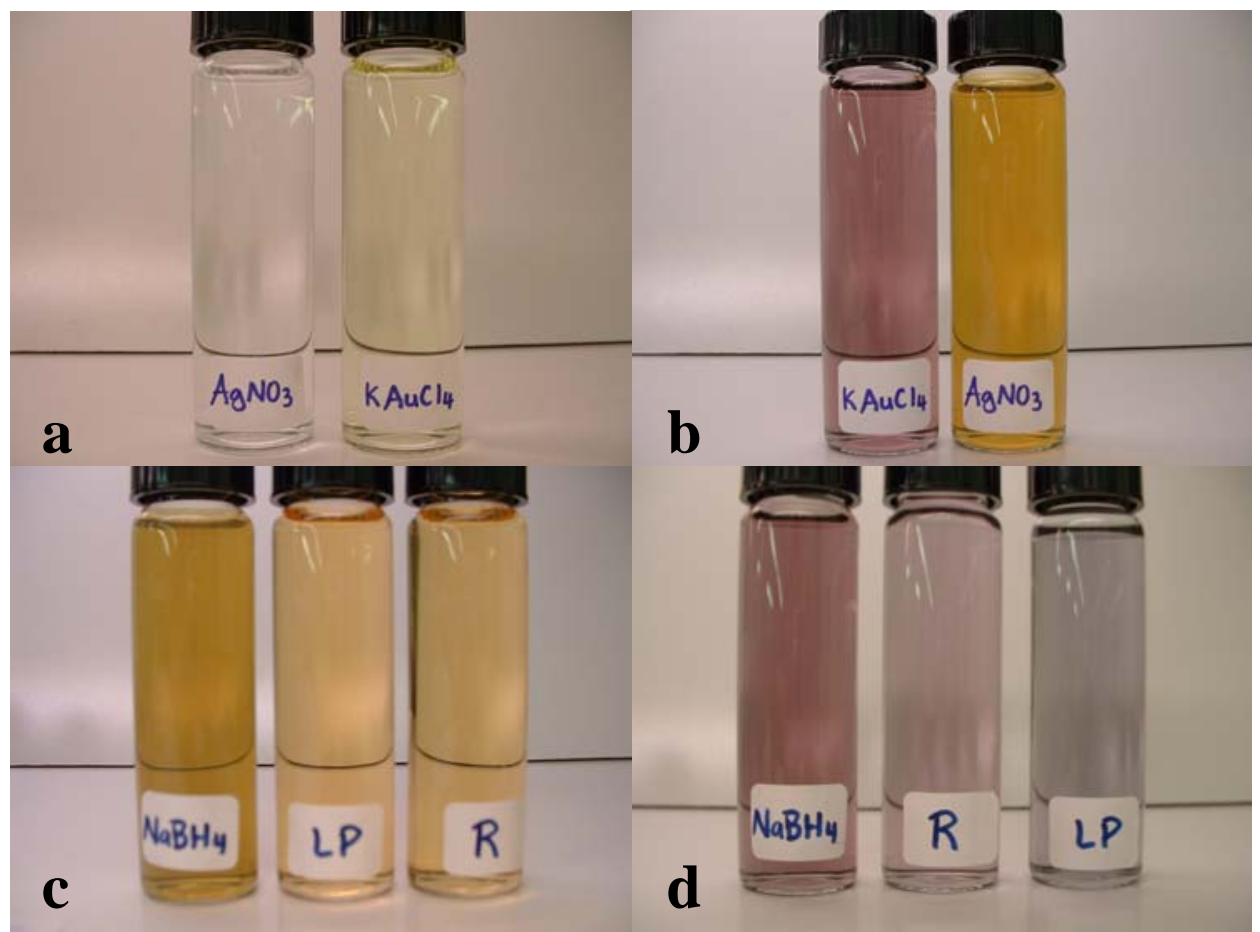

Figure 5.26. (Top) Solutions of $0.001 \mathrm{M} \mathrm{AgNO}_{3}$ and $0.001 \mathrm{M} \mathrm{KAuCl}_{4}$ shown (a) before and (b) after addition of a small amount of $\mathrm{NaBH}_{4}$. (Bottom) Solutions produced upon reaction of (c) $\mathrm{AgNO}_{3}$ and (d) $\mathrm{KAuCl}_{4}$ using either $\mathrm{NaBH}_{4}$, LP extract, or R extract as the reducing agent.

Nonetheless, the products formed by the chemical reduction of silver and gold ions using only $\mathrm{NaBH}_{4}$ were not stable in nanoparticle form. The colored solutions became darker over time and the silver and gold particles became larger and formed visible particles. These eventually fell out of solution and settled on the bottom of the vials. As the particles became visible and grew larger, the color of the solution faded from bright yellow or purple (as seen in Figure 5.26) to clear. For the reduction of $\mathrm{Ag}^{+}$, the precipitate eventually aggregated into one large spongy mass that was dark gray in color. The precipitate that developed from the reduction of gold ions was very similar in form and exhibited a dark gray color, but it also had spots that were yellow in color, presumably due to $\mathrm{Au}^{\circ}$. 
However, Jana et al. (2001a, b) reported another procedure that uses $\mathrm{NaBH}_{4}$ to reduce silver or gold ions in the presence of trisodium citrate, which acts as a capping agent. This capping agent stabilizes the particles in solution. In the literature, variations of this procedure were used to prepare either $3.5 \mathrm{~nm}$ gold seeds or $4 \mathrm{~nm}$ silver seeds. In the present study, these two techniques were carefully followed, and stable nanoparticle solutions were successfully developed. These solutions had colors identical to those initially displayed by nanoparticle solutions synthesized without the use of a capping agent, pictured in 5.26 (b). The only difference is that the solutions made using a capping agent retained their colors.

Likewise, the nanoparticles that were biologically synthesized also remain very stable over time, although chemical stabilizers are not used. Even after several months there is little or no change in appearance of the solutions, save the few samples of gold that have become contaminated. Any variation in color is negligible, and the particles remain suspended in solution. It has been speculated (Ankamwar et al. 2005, Chandran et al. 2006, Shankar et al. 2003a, 2003b, 2004a, 2004b, 2004c, 2005) that for many methods of biosynthesis of nanoparticles, certain biomolecules are also able to act as capping agents that surround the nanoparticles, providing shape control and making them stable. Thus, we see that biological synthesis of nanoparticles is able to achieve results similar to those accomplished by chemical methods. However, biosynthesis systems can produce nanoparticles using far fewer harsh chemical reagents. 


\section{Chapter 6. Summary and Conclusions}

Various plant tissue cultures, including callus culture, suspension culture, and hairy root cultures, have all been established. Suspension cultures of Stevia have been studied for uptake of silver from solution. While presence of silver inside plant cells was confirmed, it was observed that the exposure of the silver solution to suspension cultures


gold from solution was also tested, and no toxic response was observed. However, data pertaining to uptake of gold is limited.

Alfalfa and mung bean sprouts were also grown in silver solution, but again the silver caused obvious harm to the plant tissue. However, it was observed that sprouts were less sensitive to silver toxicity relative to cells in suspension cultures, which is not surprising since cells in suspension have little protection from their environment. Although uptake of silver by sprouts and plant cells has been accomplished, these systems may not be practical approaches for nanoparticle synthesis due to the detrimental effects of silver on the plant matter.

The major focus of this project is the formation of silver and gold nanoparticles by the reduction of silver and gold ions in solution using extract of Pelargonium graveolens (scented geranium). Various tests were performed and many observations were made. The central findings from these experiments are detailed in the following text. 
1. The reaction of geranium extract with $\mathrm{AgNO}_{3}$ solution to produce silver nanoparticles occurs only in the presence of light. The production of gold nanoparticles does not require light in order to react.

2. Oxygen appears to have little or no effect on reactions involving silver or gold. However, because all oxygen was not removed from the reaction environment, it cannot be concluded with confidence that oxygen does not influence the reaction.

3. By changing the length of time the extract is boiled, the composition of the extract is affected, causing it to react differently with $\mathrm{KAuCl}_{4}$ solution. Extract that has been prepared by boiling solution containing leaves and extract prepared by boiling solution after leaves have been filtered out also yield different results upon reaction with $\mathrm{KAuCl}_{4}$. Although spectrophotometry analysis shows a change in surface plasmon resonance (SPR) bands for these reactions, no distinct correlation has been identified.

4. It has been determined that $\mathrm{pH}$ has a clear impact on the reaction of geranium extract with $\mathrm{KAuCl}_{4}$ solution. By controlling the $\mathrm{pH}$ values at which the reaction is initiated, the products exhibited colors that progressively changed from dark blue to more pinkish as the $\mathrm{pH}$ was increased. It has also been observed that the $\mathrm{pH}$ changes slightly over the course of reactions involving both silver and gold solutions.

5. By using $\mathrm{NaBH}_{4}$ in the presence of trisodium citrate to chemically reduce silver and gold ions in solution, we were able to see development of colors very similar to those resulting from reduction by geranium extract. 


\section{References}

Ankamwar, B., M. Chaudhary, and M. Sastry. Gold nanotriangles biologically synthesized using tamarind leaf extract and potential application in vapor sensing. Synthesis and Reactivity in Inorganic, Metal-Organic, and Nano-Metal Chemistry. 35:19-26 (2005)

AZoNano.com news ID 2050. Scientists tackle illness with silver nanoparticles. 30 Mar. 2006. Nanotechnology News Archive. Accessed 13 June 2006. http://www.azonano.com /news.asp?newsID=2050

AZoNano.com news ID 2172. Silver nanoparticles one of the fastest growing product categories in the nanotechnology industry. 19 April 2006. Nanotechnology News Archive. Accessed 1 June 2006. http://www.azonano.com/news .asp?newsID $=2172$

Bayraktar, O. An electrophoretic tubular membrane reactor for continuous production of secondary metabolites from plant cells/tissues. Master's Thesis, West Virginia University, Morgantown. (1996)

Beach, J.A. Exploratory study on simultaneous electropermeabilization and electrophoresis. Master's Thesis, West Virginia University, Morgantown. (1998)

Chandran, S.P., M. Chaudhary, R. Pasricha, A. Ahmad, and M. Sastry. Synthesis of gold nanotriangles and silver nanoparticles using Aloe vera plant extract. Biotechnol. Prog. 22:577-583 (2006)

Colvin, V.L. The potential environmental impact of engineered nanomaterials. Nature Biotechnology 21:1166-1170 (2003)

Daniell, H., S.J. Streatfield, and K. Wycoff. Medical molecular farming: production of antibodies, biopharmaceuticals and edible vaccines in plants. TRENDS in Plant Science 6:219-225 (2001)

Doran, P.M. Properties and applications of hairy root cultures. In Plant Biotechnology and Transgenic Plants, 143-161. K. Oksman-Caldentey and W.H. Barz, Eds., Marcel Dekker, New York. (2002)

Elechiguerra, J.L., J.L. Burt, J.R. Morones, A.Camacho-Bragado, X. Gao, H.H. Lara, and M.J. Yacaman. Interaction of silver nanoparticles with HIV-1. Journal of Nanobiotechnology 3 (2005) 
Franklin, C.I. and R.A. Dixon. Initiation and maintenance of callus and cell suspension cultures. In Plant Cell Culture: A Practical Approach, 1-25. R.A. Dixon and R.A. Gonzales. Eds., Oxford University Press, New York. (1994)

Gardea-Torresdey, J.L., E. Gomez, J.R. Peralta-Videa, J.G. Parsons, H. Troiani, and M. Jose-Yacaman. Alfalfa sprouts: a natural source for the synthesis of silver nanoparticles. Langmuir 19:1357-1361 (2003)

Gardea-Torresdey, J.L., J.G. Parsons, E. Gomez, J. Peralta-Videa, H.E. Troiani, P. Santiago, and M.J. Yacaman. Formation and growth of Au nanoparticles inside live alfalfa plants. Nano Letters 2:397-401 (2002)

Gardea-Torresdey, J.L., K.J. Tiemann, G. Gamez, K. Dokken, and M.J. Yacaman. Innovative technology to recover gold(III) from aqueous solutions by using Medicago sativa (alfalfa). Proceedings of the 1998 Conference on Hazardous Waste Research. (1998)

Gupta, R., S. Banerjee, G.R. Mallavarapu, S. Sharma, S.P.S. Khanuja, A.K. Shasany, and S. Kumar. Development of a superior somaclone of rose-scented geranium and a protocol for inducing variants. HortScience 37:632-636 (2002)

Höxtermann, E. Cellular 'elementary organisms' in vitro. The early vision of Gottlieb Haberlandt and its realization. Physiol. Plant. 100:716-728 (1997)

Hood, E.E. and S.L. Woodard. Industrial proteins produced from transgenic plants. In Plants as Factories for Protein Production, 119-135. E.E. Hood and J.A. Howard, Eds., Kluwer Academic Publishers, The Netherlands. (2002)

Jana, N.R., L. Gearheart, and C.J. Murphy. Wet chemical synthesis of high aspect ratio cylindrical gold nanorods. J. Phys. Chem. 105:4065-4067 (2001a)

Jana, N.R., L. Gearheart, and C.J. Murphy. Wet chemical synthesis of silver nanorods and nanowires of controllable aspect ratio. Chem. Commun. 617-618 (2001b)

Kim, F., J.H. Song, and P Yang. Photochemical synthesis of gold nanorods. J. Am. Chem. Soc. 124:14316-14317 (2002)

KrishnaRaj, S., Y. Bi, and P.K. Saxena. Somatic embryogenesis and Agrobacteriummediated transformation system for scented geraniums (Pelargonium sp. 'Frensham'). Planta 201:434-440 (1997)

Lakshmana Rao, P.V. In vitro plant regeneration of scented-leaved geranium Pelargonium graveolens. Plant Sci. 98:193-198 (1994) 
Larrick, J.W., L. Yu, C. Naftzger, S. Jaiswal, and K. Wycoff. Human pharmaceuticals produced in plants. In Plants as Factories for Protein Production, 79-101. E.E. Hood and J.A. Howard, Eds., Kluwer Academic Publishers, The Netherlands. (2002)

Liz-Marzán, L.M. Nanometals: formation and color. Materials Today 7:26-31 (2004)

Megeji, N.W., J.K. Kumar, V. Singh, V.K. Kaul, and P.S. Ahuja. Introducing Stevia rebaudiana, a natural zero-calorie sweetener. Current Science 88:801-804 (2005)

Mitchell, D.N., H.A. Godwin, and E. Claudio. Nanoparticle toxicity in Saccharomyces cerevisiae: a comparative study using Au colloid, Ag colloid, and $\mathrm{HAuCl}_{4} \cdot 3 \mathrm{H}_{2} \mathrm{O}$ in solution. Nanoscape 1:59-69 (2004)

Murphy, C.J. and N.R. Jana. Controlling the aspect ratio of inorganic nanorods and nanowires. Advanced Materials 14:80-82 (2002)

Penn, C. Media and Growth. In Handling Laboratory Microorganisms, 33-40. Open University Press, Milton Keynes. (1991)

Rai, A., A. Singh, A. Ahmad, and M. Sastry. Role of halide ions and temperature on the morphology of biologically synthesized gold nanotriangles. Langmuir 22:736741 (2006)

Rao, P.V.L. In vitro plant regeneration of scented-leaved geranium Pelargonium graveolens. Plant Science 98: 193-198 (1994)

Robins, R.J. Secondary products from cultures cells and organs: I. Molecular and cellular approaches. In Plant Cell Culture: A Practical Approach, 169-198. R.A. Dixon and R.A. Gonzales. Eds., Oxford University Press, New York. (1994)

Savita, S.M., K. Sheela, S. Sunanda, A.G. Shankar, and P. Ramakrishna. Stevia rebaudiana - a functional component for food industry. J. Hum. Ecol. 15:261-264 (2004)

Saxena, G., S. Banerjee, L. Rahman, G.R. Mallavarapu, S. Sharma, and S. Kumar. An efficient in vitro procedure for micropropagation and generation of somaclones of rose scented Pelargonium. Plant Sci. 155:133-140 (2000)

Scragg, A.H. Secondary products from cultures cells and organs: II. Large scale culture. In Plant Cell Culture: A Practical Approach, 199-224. R.A. Dixon and R.A. Gonzales. Eds., Oxford University Press, New York. (1994)

Shankar, S.S., A. Ahmad, R. Pasricha, and M. Sastry. Bioreduction of chloroaurate ions by geranium leaves and endophytic fungus yields gold nanoparticles of different shapes. Journal of Materials Chemistry 13:1822-1826 (2003a) 
Shankar, S.S., A. Ahmad, and M. Sastry. Geranium leaf assisted biosynthesis of silver nanoparticles. Biotechnol. Prog. 19:1627-1631 (2003b)

Shankar, S.S., A. Rai, A. Ahmad, and M. Sastry. Biosynthesis of silver and gold nanoparticles from extracts of different parts of the geranium plant. Applied Nanoscience 1:69-77 (2004a)

Shankar, S.S., A. Rai, A. Ahmad, and M. Sastry. Rapid synthesis of Au, Ag, and bimetallic Au core-Ag shell nanoparticles using neem (Azadirachta indica) leaf broth. 275:496-502 (2004b)

Shankar, S.S., A. Rai, B. Ankamwar, A. Singh, A.Ahmad, and M. Sastry. Biological synthesis of triangular gold nanoprisms. Nature Materials 3:482-488 (2004c)

Shankar, S.S., A. Rai, A. Ahmad, and M. Sastry. Controlling the optical properties of lemongrass extract synthesized gold nanotriangles and potential application in infrared-absorbing optical coatings. Chem. Mater. 17:566-572 (2005)

Sivarum, L. and U. Mukundan. In vitro culture studies on Stevia rebaudiana. In Vitro Cell. Dev. Biol.-Plant 39:520-523 (2003)

Swanson, S.M., G.B. Mahady, and C.W.W. Beecher. Stevioside biosynthesis by callus, root, shoot and rooted-shoot cultures in vitro. Plant Cell, Tissue and Organ Culture 28:151-157 (1992)

University of Iowa Healthcare. Sugar. 5 June 2006. Nature's Pharmacy: Ancient Knowledge, Modern Medicine. Accessed 15 Oct. 2006. http://www.uihealthcare.com/depts/medmuseum/galleryexhibits/naturespharmacy /sugar/sugar.html

Yamazaki, T. and H.E. Flores. Examination of steviol glucosides production by hairy root and shoot cultures of Stevia rebaudiana. Journal of Natural Products 54:986992 (1991) 


\section{Appendices}

\section{Appendix A. Methods of Sanitization}

Aseptic conditions are necessary for preventing contamination of plant cultures. The laboratory must be cleaned regularly, and all glassware, tools, and media used in culture procedures must be sterilized.

\section{A.1 Laboratory}

The lab is the first factor that must be considered. Bacteria and fungi can become airborne, and can infect the plant tissue cultures even if aseptic techniques have been used throughout the culture procedure. Thus, efforts must be made to keep all surfaces free of germs. A disinfectant cleaner was used to wipe down these surfaces. This process was repeated as needed to keep dust off of the countertops and floor. Also, a disinfectant spray was also used occasionally to kill airborne microbes.

\section{A.2 Laminar Flow Hood}

All procedures involving preparation of media, inoculation of plant cells and tissues, and subculturing of cultures were performed in the laminar flow hood. Prior to each use, the hood was cleaned thoroughly using $70 \% \mathrm{v} / \mathrm{v}$ ethanol solution. All 5 hood surfaces including the aluminum screen were saturated by spraying with the solution and then wiped down with a paper towel. Airflow was then turned on and the hood was left to dry before use. The filter in the hood is designed to remove any contaminants out of the air, so objects placed on the work surface are protected from infection. 


\section{A.3 Incubator}

The incubator, which controls temperature and light conditions for the plant tissue cultures, was wiped clean inside approximately every month, or as needed, with $70 \% \mathrm{v} / \mathrm{v}$ ethanol. The ethanol was periodically sprayed into the air as well.

\section{A.4 Hands}

Hands were washed thoroughly with soap and warm water. Then the $70 \%$ ethanol solution was sprayed onto skin any time a process required aseptic conditions. This was repeated several times throughout the procedure, especially after any unsanitary object was touched.

\section{$\underline{\text { A.5 Glassware }}$}

Glassware used to prepare the medium was sterilized by one of two methods: dry heat or steam. Either way, the glassware was first washed in the sink with soap and water to remove any visible dirt or chemicals. For the dry heat method, the glassware was then placed in an oven at $160^{\circ} \mathrm{C}$ for 3-4 hours. Alternatively, steam sterilizing was used when several hours preparation time was not available. In this case, the glassware was put in the autoclave at $120^{\circ} \mathrm{C}$ for $15 \mathrm{~min}$.

\section{$\underline{\text { A.6 Tools }}$}

Instruments used in culturing and subculturing must also be thoroughly disinfected, since they come in direct contact with the plant tissue that is to be protected from 
contamination. To accomplish this, utensils were wiped down and soaked in a $90 \% \mathrm{v} / \mathrm{v}$ ethanol solution. Then they were placed over a flame to burn off the alcohol and were allowed to cool before use. The instruments were repeatedly dipped and flamed throughout the procedure. With both the alcohol and the flame working as disinfectants, any microbial contaminants will likely be eliminated.

\section{A.7 Medium}

Media used were steam sterilized in the autoclave. The flask containing the medium was covered with perforated aluminum foil before being placed in the steam sterilizer. Controls were set to heat to a temperature of $120^{\circ} \mathrm{C}$ for $15 \mathrm{~min}$.

\section{A.8 Seeds and Explants}

Leaf or stem explants were first washed thoroughly in warm water and soap. In some cases, the explants were then dipped in $70 \%$ ethanol for 30 seconds. Then they were transferred to the Fisher FS6 ultrasonic cleaner, which contained a solution of dilute bleach (1\%-2\%) and 1-3 drops of Tween 20 per $100 \mathrm{ml}$. The bleach solution was mixed using commercial bleach, which is typically available in a $5.25 \%(\mathrm{w} / \mathrm{v})$ concentration. It was further diluted to produce a solution with a concentration lying between $1 \%$ and $2 \%$. The explants remained in the ultrasonic cleaner for 10-15 min. Afterward, explants were removed and rinsed several times in distilled water that had been sterilized in the autoclave and cooled to room temperature in the laminar flow hood. 
To grow sterile plantlets, seeds were prepared in a similar manner, but were soaked in a $70 \%$ ethanol solution for 5 minutes before transferring to the ultrasonic cleaner. The ultrasonic cleaner again contained a dilute bleach solution (1\%-2\%) and Tween 20, and was turned on for 10 minutes. The seeds were rinsed 6 times with sterile water before being placed onto the agar medium.

To prepare the mung bean and alfalfa seeds for sprouters, the seeds were soaked in a solution of ZeroTol (50X dilution) for 15-30 minutes. Then they were rinsed several times before use. 


\section{Appendix B. Procedures for Media Preparation}

\section{B.1 General Procedure for Preparation of Solid Media}

1. Wash all glassware with soap and water. Then rinse in deionized/distilled (DD) water.

2. Sterilize baby jars or petri dishes using techniques described in Appendix A and place them in the laminar flow hood while medium is being prepared.

3. Prepare the medium in a clean $1000 \mathrm{ml}$ Erlenmeyer flask. First, fill the flask with $800 \mathrm{ml}$ unsterilized DD water.

4. Now add components as indicated in Table B.1 (p. 81).

5. Bring the volume to $1000 \mathrm{ml}$ by adding more $\mathrm{DD}$ water.

6. Place the mixture on a stirring hot plate. Set stirring to $250 \mathrm{rpm}$. After well mixed, adjust $\mathrm{pH}$ by adding either $1.0 \mathrm{~N} \mathrm{KOH}$ or $0.5 \mathrm{~N} \mathrm{HCl}$ dropwise.

7. Set the temperature to $150^{\circ} \mathrm{C}$. After ten minutes increase temperature to $225^{\circ} \mathrm{C}$. Ten minutes later, raise the temperature again to $275^{\circ} \mathrm{C}$. Allow the medium to remain on the hot plate until it turns clear.

8. Cover the medium with foil, and pierce small holes into the foil with a pen or paperclip. Place in the autoclave and sterilize it at $120^{\circ} \mathrm{C}$ for $15 \mathrm{~min}$. Baby jar lids may also be placed separately in the autoclave at this time.

9. Pour the sterilized medium into the jars or petri dishes and cover with appropriate lids. Allow the medium to cool and solidify before use. If excessive condensation develops inside the dish or jar, tilt the lid so air can flow in and reduce the moisture levels (Penn 1991). Covered containers of media may also be refrigerated for future use. 


\section{B.2 General Procedure for Preparation of Liquid Media}

1. Wash all glassware with soap and water. Then rinse in deionized/distilled (DD) water.

2. Prepare the medium in a clean $1000 \mathrm{ml}$ Erlenmeyer flask. First, fill the flask with $800 \mathrm{ml}$ unsterilized DD water.

3. Now add components as indicated in Table B.1 (p. 81), but omit agar.

4. Bring the volume to $1000 \mathrm{ml}$ by adding more DD water.

5. Place the mixture on a stirring hot plate. Set stirring to $250 \mathrm{rpm}$. After medium has been thoroughly mixed, adjust $\mathrm{pH}$ by adding either $1.0 \mathrm{~N} \mathrm{KOH}$ or $0.5 \mathrm{~N} \mathrm{HCl}$ dropwise.

6. Pour the medium into $250 \mathrm{ml}$ or $500 \mathrm{ml}$ Erlenmeyer flasks and cover with BugStoppers ${ }^{\mathrm{TM}}$ or cotton balls. For $250 \mathrm{ml}$ flasks, use about $125 \mathrm{ml}$ of medium, and for $500 \mathrm{ml}$ flasks, fill the medium to a volume of about $175 \mathrm{ml}$. Place in the autoclave and sterilize them at $120^{\circ} \mathrm{C}$ for $15 \mathrm{~min}$.

7. Allow medium to cool to room temperature before use. Refrigerate excess medium for future use.

\section{B.3 Preperation of Kinetin Solution}

1. Weigh out $10 \mathrm{mg}$ of kinetin and place in a clean, dry $250 \mathrm{ml}$ beaker.

2. Add 1-2 $\mathrm{ml}$ of $1.0 \mathrm{~N} \mathrm{KOH}$ and stir until kinetin is dissolved.

3. Bring the volume up to $100 \mathrm{ml}$ with DD water.

4. Cover with Parafilm ${ }^{\circledR}$ and store in the refrigerator until use. 


\section{B.4 Preparation of 2,4-Dichlorophenoxyacetic Acid (2,4-D) Solution}

1. Weigh $2.2 \mathrm{mg}$ of 2,4-D and transfer to a clean $250 \mathrm{ml}$ beaker.

2. Add 1-2 $\mathrm{ml} 1.0 \mathrm{~N} \mathrm{KOH}$ and swirl beaker until solid has dissolved.

3. Add DD water to bring the volume up to $100 \mathrm{ml}$.

4. Cover with Parafilm ${ }^{\circledR}$ and store solution in the refrigerator until needed. 
Table B.1. Components required for preparation of various media

\begin{tabular}{|c|c|c|c|c|c|c|c|c|c|c|}
\hline Type of Medium & $\begin{array}{l}\text { MS } \\
\text { Medium }\end{array}$ & $\begin{array}{l}\text { MS } \\
\text { Medium }\end{array}$ & $\begin{array}{l}1 / 2 \mathrm{MS} \\
\text { Medium }\end{array}$ & $\begin{array}{l}1 / 2 \text { MS } \\
\text { Medium } \\
\text { with } \\
\text { antibiotic }\end{array}$ & $\begin{array}{l}\text { Gamborg's } \\
\text { B5 } \\
\text { Medium }\end{array}$ & $\begin{array}{l}\text { Gamborg's } \\
1 / 2 \mathrm{~B} 5 \\
\text { Medium } \\
\text { with } \\
\text { antibiotic }\end{array}$ & $\begin{array}{l}\text { A2 } \\
\text { Medium }\end{array}$ & $\begin{array}{l}\text { MS (a) } \\
\text { Medium }\end{array}$ & $\begin{array}{l}\text { MS (b) } \\
\text { Medium }\end{array}$ & $\begin{array}{l}\text { Yeast } \\
\text { Mannitol } \\
\text { Broth } \\
\text { (YMB) }\end{array}$ \\
\hline Uses & $\begin{array}{l}\text { periwinkle } \\
\text { callus and } \\
\text { suspension }\end{array}$ & $\begin{array}{l}\text { Stevia } \\
\text { callus and } \\
\text { suspension }\end{array}$ & $\begin{array}{l}\text { Stevia } \\
\text { plantlets } \\
\text { and } \\
\text { hairy } \\
\text { roots }\end{array}$ & $\begin{array}{l}\text { Stevia } \\
\text { hairy } \\
\text { roots }\end{array}$ & $\begin{array}{l}\text { periwinkle } \\
\text { plantlets }\end{array}$ & $\begin{array}{l}\text { periwinkle } \\
\text { hairy roots }\end{array}$ & $\begin{array}{l}\text { beet callus } \\
\text { and } \\
\text { suspension }\end{array}$ & $\begin{array}{l}\text { geranium } \\
\text { callus } \\
\text { started } \\
\text { from } \\
\text { leaves }\end{array}$ & $\begin{array}{l}\text { geranium } \\
\text { callus } \\
\text { started } \\
\text { from } \\
\text { stems } \\
\end{array}$ & $\begin{array}{l}A \text {. } \\
\text { rhizogenes }\end{array}$ \\
\hline $\mathrm{PH}$ & $5.7-5.8$ & $5.7-5.8$ & 5.7 & 5.7 & 5.5 & 5.5 & 5.5 & 5.6 & 5.8 & 6.8 \\
\hline Salt Base (1pk/L) & $\begin{array}{l}\text { MS with } \\
\text { vitamins }\end{array}$ & $\begin{array}{l}\text { MS with } \\
\text { vitamins }\end{array}$ & $\begin{array}{l}1 / 2 \mathrm{pk} \\
\mathrm{MS} \\
\text { with } \\
\text { vitamins }\end{array}$ & $\begin{array}{l}1 / 2 \mathrm{pk} \text { MS } \\
\text { with } \\
\text { vitamins }\end{array}$ & $\begin{array}{l}\text { Gamborg's } \\
\text { B5 with } \\
\text { vitamins }\end{array}$ & $\begin{array}{l}\text { 1/2 pk } \\
\text { Gamborg's } \\
\text { B5 with } \\
\text { vitamins }\end{array}$ & $\begin{array}{l}\text { Gamborg's } \\
\text { B5 with } \\
\text { vitamins }\end{array}$ & $\begin{array}{l}\text { MS with } \\
\text { vitamins }\end{array}$ & $\begin{array}{l}\text { MS with } \\
\text { vitamins }\end{array}$ & - \\
\hline Sucrose & $30 \mathrm{~g}$ & $30 \mathrm{~g}$ & $20 \mathrm{~g}$ & $20 \mathrm{~g}$ & $20 \mathrm{~g}$ & $20 \mathrm{~g}$ & $20 \mathrm{~g}$ & $30 \mathrm{~g}$ & $30 \mathrm{~g}$ & - \\
\hline Agar & $8 \mathrm{~g}$ & $9 \mathrm{~g}$ & $4 \mathrm{~g}$ & $4 \mathrm{~g}$ & $6 \mathrm{~g}$ & $6 \mathrm{~g}$ & $6 \mathrm{~g}$ & $8 \mathrm{~g}$ & $8 \mathrm{~g}$ & - \\
\hline Kinetin solution & $10 \mathrm{ml}$ & - & - & - & - & - & $0.5 \mathrm{ml}$ & $5 \mathrm{ml}$ & $\begin{array}{l}10 \mathrm{mg} \\
(100 \mathrm{ml})\end{array}$ & - \\
\hline 2,4 D solution & $10 \mathrm{ml}$ & - & - & - & - & - & $1.0 \mathrm{ml}$ & $50 \mathrm{ml}$ & - & - \\
\hline $\begin{array}{l}1- \\
\text { Naphthaleneacatic } \\
\text { Acid (NAA) }\end{array}$ & - & $0.5 \mathrm{mg}$ & - & - & - & - & - & $1 \mathrm{mg}$ & $1.0 \mathrm{mg}$ & - \\
\hline $\begin{array}{l}\text { 6- } \\
\text { Benzylaminopurine }\end{array}$ & - & $0.5 \mathrm{mg}$ & - & - & - & - & - & - & - & - \\
\hline Ampicillin & - & - & - & $0.5 \mathrm{~g}$ & - & 0.25 & - & - & - & - \\
\hline $\mathrm{K}_{2} \mathrm{HPO}_{4}$ & - & - & - & - & - & - & - & - & - & $0.5 \mathrm{~g}$ \\
\hline $\mathrm{MgSO}_{4} \cdot 2 \mathrm{H}_{2} \mathrm{O}$ & - & - & - & - & - & - & - & - & - & $0.2 \mathrm{~g}$ \\
\hline $\mathrm{NaCl}$ & - & - & - & - & - & - & - & - & - & $0.1 \mathrm{~g}$ \\
\hline Yeast extract & - & - & - & - & - & - & - & - & - & $0.4 \mathrm{~g}$ \\
\hline Mannitol & - & - & - & - & - & - & - & - & - & $5-10 \mathrm{~g}$ \\
\hline
\end{tabular}




\section{Appendix C. Procedures for Induction and Maintenance of Callus Cultures}

\section{$\underline{\text { C.1 Induction and Maintenance of Periwinkle Callus Culture }}$}

1. Prepare solid MS medium for periwinkle as described in Appendix B and place in the laminar flow hood for several hours to allow it to reach room temperature.

2. Select a few healthy leaves from the plant and wash thoroughly with warm water and soap.

3. Transfer leaves to an ultrasonic cleaner containing a dilute bleach solution $(1 \%$ 2\%) and 2-3 drops of Tween 20 per $100 \mathrm{ml}$. Commercial bleach is typically available in a $5.25 \%(\mathrm{w} / \mathrm{v})$ concentration, so it must be further diluted to produce a solution with a concentration lying between $1 \%$ and $2 \%$. Allow the explants to remain in the ultrasonic cleaner for $10 \mathrm{~min}$.

4. After removing from the ultrasonic cleaner with sterile tweezers, move the sterile leaves to the laminar flow hood.

5. Rinse the bleach solution from the leaves using sterilized DD water.

6. Cut leaves into small pieces using either a cork borer or sharp scissors. Dip all utensils in ethanol and flame to prevent contamination. Avoid using leaf cuttings with veins.

7. Place cuttings on the agar surface, 3 or 4 per jar or petri dish, with the top of the leaf facing up. Apply a little pressure to ensure good contact of the leaf cuttings with the medium.

8. Place cultures in an incubator to keep the temperature at $25^{\circ} \mathrm{C}$. Set the photoperiod to have 16 continuous hours of daily light. 
9. Callus will appear after about 2 weeks. After a period of 4 weeks, cut the leaf away from the callus, and place into new jars with fresh medium.

10. Continue subculturing at 2-3 week intervals, transferring only the healthiest and most friable portions of callus to fresh medium.

\section{C.2 Induction and Maintenance of Stevia Callus Culture}

1. According to the directions given in Appendix B, prepare solid MS medium specially formulated for Stevia and allow to reach room temperature in the laminar flow hood.

2. Select a few healthy leaves from the plant and wash thoroughly with tap water.

3. Transfer leaves to an ultrasonic cleaner containing a dilute bleach solution (1\%2\%) and 2-3 drops of Tween 20 per $100 \mathrm{ml}$. Dilute commercial bleach to produce a solution with a concentration lying between $1 \%$ and $2 \%$. Allow the explants to remain in the ultrasonic cleaner for $10 \mathrm{~min}$.

4. Use sterile tweezers to remove leaves from the ultrasonic cleaner, and transfer them to the laminar flow hood.

5. Rinse the bleach solution from the leaves using sterilized DD water.

6. Cut leaves into small pieces using either a cork borer or sharp scissors. Dip all utensils in $90 \% \mathrm{v} / \mathrm{v}$ ethanol and flame to prevent contamination. Avoid using leaf cuttings with veins.

7. Place cuttings on the agar surface, 3 or 4 per jar or petri dish, with the top of the leaf facing up. Apply a little pressure to ensure good contact of the cuttings with the medium. 
8. Place jars or petri dishes in an incubator to keep the temperature at $27^{\circ} \mathrm{C}$ and allow constant exposure to light.

9. Callus will appear from the cut edges of the leaves after about 2 weeks. After a period of about 6 weeks, cut the leaf away from the callus, and place into new jars with fresh MS medium.

10. Conduct subcultures every 4-5 weeks, transferring only the greenest and most viable portions of callus to fresh medium.

\section{$\underline{\text { C.3 Induction and Maintenance of Geranium Callus Culture Using Leaf Explants }}$}

1. According to the directions given in Appendix B, prepare MS solid medium specially formulated for inducing geranium callus using leaves (designated as MS(a) medium). Allow medium to warm up (if taken from the refrigerator) or cool down (if freshly prepared) in the laminar flow hood to reach room temperature.

2. Select the youngest uncurled leaves from the plant and wash thoroughly with tap water. To sterilize leaves, dip for 10 seconds in $70 \%$ ethanol, and then transfer them to an ultrasonic cleaner containing a solution of $1.4 \%$ bleach and 1 drop Tween 20 per $100 \mathrm{ml}$.

3. After 15 minutes, remove leaves from the ultrasonic cleaner, and transfer them to the laminar flow hood.

4. Rinse the bleach solution from the leaves using sterilized DD water.

5. Cut leaves into small pieces using sharp scissors. Dip all utensils in $90 \% \mathrm{v} / \mathrm{v}$ ethanol and flame to prevent contamination. 
6. Place cuttings on the agar surface, 3 or 4 per jar or petri dish, with the underside of the leaf facing down towards the agar. Apply a little pressure to ensure good contact of leaves with the medium.

7. Place jars or petri dishes in an incubator to keep the temperature at $25^{\circ} \mathrm{C}$. Set the light cycle to 16 hours light/8 hours dark.

8. Callus may not appear until over 2 months later. After callusing is observed, place leaves with callus into new jars with fresh MS medium.

\section{C.4 Induction and Maintenance of Geranium Callus Culture Using Stem Explants}

1. According to the directions given in Appendix B, prepare MS solid medium specially formulated for inducing geranium callus using stem segments (MS(a) medium). Allow medium to warm up (if taken from the refrigerator) or cool down (if freshly prepared) in the laminar flow hood to reach room temperature.

2. Cut the youngest tips of stems from the plant and wash thoroughly with tap water. Dip the segments in $70 \%$ ethanol for 10 seconds and place them into an ultrasonic cleaner containing a solution of $1 \%-2 \%$ bleach and 2-3 drops of Tween 20 per $100 \mathrm{ml}$. Sterilize the segments in the ultrasonic cleaner for 15 minutes.

3. Remove stem explants from the ultrasonic cleaner, and transfer them to the laminar flow hood.

4. Rinse the bleach solution from the leaves using sterilized DD water.

5. Cut stems into segments ranging from 1.5 to $2 \mathrm{~cm}$ long using sharp scissors. Dip all utensils in $90 \% \mathrm{v} / \mathrm{v}$ ethanol and flame to prevent contamination. 
6. Incubate the stem segments vertically, inserting the basal portion of the explant under the surface of the agar. Use 1 or 2 explants per baby jar of medium.

7. Place lids on cultures and place in an incubator to keep the temperature at $25^{\circ} \mathrm{C}$. Set the photoperiod for 16 hours light/8 hours dark.

8. Callus will appear approximately 3-4 weeks later. After sufficient callusing is observed, excise the callus from the stem and subculture into new jars with fresh MS medium.

\section{C.5 Maintenance of Beet Callus}

1. Prepare solid A2 medium (Appendix B). Place it in the laminar flow hood and allow it to reach room temperature.

2. Soak all utensils in $90 \%$ ethanol and flame before use. Repeat periodically throughout procedure.

3. Use sterilized tweezers to transfer small pieces of callus (at least $1 \mathrm{~cm}$ in diameter) to fresh A2 medium. Use only the reddest, most friable parts of callus.

4. Replace lids on cultures and incubate them under $16 \mathrm{hrs}$ light/8 hrs dark. Maintain cultures at a temperature of approximately $25^{\circ} \mathrm{C}$.

5. Conduct following subcultures at intervals of 3-4 weeks. 


\section{Appendix D. Procedures for Establishment and Maintenance of Suspension Cultures}

\section{D.1 Establishment and Maintenance of Stevia Suspension Culture}

1. Prepare liquid MS medium for Stevia as described in Appendix B.

2. Separate the medium into $500 \mathrm{ml}$ Erlenmeyer flasks, filling each with approximately $200 \mathrm{ml}$. Cap each flask with a BugStopper ${ }^{\mathrm{TM}}$ or a cotton ball.

3. Sterilize flasks in an autoclave at $120^{\circ} \mathrm{C}$ for 15 minutes.

4. Remove flasks from the autoclave and transfer them directly to the laminar flow hood.

5. When medium has cooled to room temperature, transfer callus from solid MS medium in baby jars to the liquid MS medium in the flasks. Dip a scalpel or tweezers in $90 \%$ ethanol and flame before removing the most friable and viable portions of callus for initiation of suspension cell culture. Add about 3-5 grams of callus to each flask, and cut large chunks into small pieces to increase surface area for nutrient absorption.

6. Place flasks on an orbital shaker $(100-120 \mathrm{rpm})$ at $27^{\circ} \mathrm{C}$ under 24 hours constant fluorescent lighting.

7. After 10-14 days, transfer cell cultures to fresh medium. Remove large and discolored pieces, and swirl flasks for mixing of cells. Then transfer $100 \mathrm{ml}$ of suspension to a new flask containing $100 \mathrm{ml}$ fresh medium, to attain a 1:1 ratio.

8. Conduct subsequent subcultures every 10-14 days. At this point cell density is much higher, so a ratio of approximately 1:7 $(25 \mathrm{ml}$ old:175 $\mathrm{ml}$ fresh) can be used. 


\section{D.2 Establishment and Maintenance of Beet Suspension Culture}

1. Prepare liquid A2 medium for beets as described in Appendix B.

2. Follow procedures as described in steps 2-4 of D.1.

3. When medium has cooled to room temperature, transfer callus from solid A2 medium in baby jars to the liquid A2 medium in the flasks. Dip a scalpel or tweezers in $90 \%$ ethanol and flame before removing the most friable and viable portions of callus for initiation of suspension cell culture. Add about 3-5 grams of callus to each flask, and cut large chunks into small pieces to increase surface area for nutrient absorption.

4. Place flasks on an orbital shaker $(100-120 \mathrm{rpm})$ at $25^{\circ} \mathrm{C}$ with 16 hours fluorescent lighting daily.

5. After 7-10 days, transfer cell cultures to fresh medium. Remove large and discolored pieces, and swirl flasks for mixing of cells. Then transfer $100 \mathrm{ml}$ of suspension to a new flask containing $100 \mathrm{ml}$ fresh medium, to attain a 1:1 ratio.

6. Conduct subsequent subcultures every 7-10 days. When cell density is sufficiently high, a ratio of approximately 1:7 (25 $\mathrm{ml}$ old:175 $\mathrm{ml}$ fresh) can be used.

\section{D.3 Establishment and Maintenance of Periwinkle Suspension Culture}

1. Prepare liquid MS medium for periwinkle as described in Appendix B.

2. Follow procedures exactly as described in steps 2-8 of D.1, except in step 6 place flasks under 16 hours fluorescent lighting. 


\section{Appendix E. Procedure for Growing Suspension Cultures in $\mathbf{A g N O}_{3}$ Solution}

1. Add silver solution to the suspension at the midpoint of its growth period. For stevia and periwinkle suspension, the subculture period is 14 days. Thus, introduce the silver solution on day 7. For beet suspension, the growth period is 10 days, so add silver solution on day 5.

2. Prepare an $\mathrm{AgNO}_{3}$ solution with an appropriate $\mathrm{Ag}^{+}$concentration. Obtain a clean volumetric flask with an appropriate volume (usually $50 \mathrm{ml}$ ). Rinse with DD water. Carefully weigh the specified amount of $\mathrm{AgNO}_{3}$ and transfer to the volumetric flask. Fill the flask with DD water. Stir the solution for several minutes to ensure homogeneity.

3. Under aseptic conditions in the laminar flow hood, filter sterilize the $\mathrm{AgNO}_{3}$ solution using a Millipore membrane (See Appendix F).

4. Using two sterile $100 \mathrm{ml}$ volumetric flasks, precisely measure $200 \mathrm{ml}$ of suspension. Discard any extra, but pour the $200 \mathrm{ml}$ back into the flask or into a new sterile flask.

5. Use a sterile pipet to deliver the correct amount of $\mathrm{AgNO}_{3}$ solution to the suspension or hairy root culture, so that the $\mathrm{Ag}^{+}$concentration is appropriate at the final volume.

6. Incubate the suspension at normal light and temperature conditions.

7. Harvest Stevia and periwinkle cells 7 days after introduction of the silver solution (14 days from previous subculture), and harvest beet cells 5 days after introducing silver solution (10 days from the previous subculture). At this time, filter cells 
from solution under vacuum filtration using Whatman \#1 qualitative filter paper. Wash cells 6 times with $200 \mathrm{ml}$ of DD water to remove any silver solution from the outside of the biomass. Tap water should not be used, $\mathrm{as}^{+} \mathrm{g}^{+}$will react with the $\mathrm{Cl}^{-}$found in tap water to form a precipitate.

8. Place washed cells on a tray of aluminum foil in the Fisher Isotemp ${ }^{\mathrm{TM}}$ Oven (100 series model). Dry cells overnight at a temperature of approximately $80^{\circ} \mathrm{C}$.

9. Allow cells to cool, and record dry weight.

10. Transfer cells into a clean, dry crucible and burn at $700^{\circ} \mathrm{C}$ in a muffle furnace (FB14415M, Barnstead/Thermolyne Corp.) for about $2.5 \mathrm{hrs.}$

11. After burning, allow ash to cool. Then weigh again and record weight.

12. Store ash in clean, dry containers and place samples in a dessicator until needed.

13. Analyze ash for silver content using an Atomic Absorption Spectrophotometer (See Appendix I). 


\section{Appendix F. Filter Sterilization Using Millipore Membranes}

1. Steam sterilize glassware and equipment for vacuum filtration for 15 minutes at $120^{\circ} \mathrm{C}$ in the autoclave.

2. Remove the sterilized apparatus from the autoclave and place in the laminar flow hood. If a precise concentration is required for the solution that needs to be filter sterilized, allow time for the apparatus to dry before proceeding.

3. Take a Millipore membrane (pore size $2.2 \mu \mathrm{m}$ ) from the refrigerator and assemble the vacuum filtration unit. The shiny side of the membrane must be facing down, toward the filtrate. Ensure that all connections are sealed and keep the vacuum pump near the laminar flow hood, so the tube stretching from the flask to the pump will not be tightly pulled.

4. Turn on the vacuum pump.

5. Pour the solution through the Millipore membrane to render it sterile. 


\section{Appendix G. Procedures for Growing Mung Bean and Alfalfa Sprouts in Silver Solution}

\section{$\underline{\text { G.1 Procedure for Growing Mung Bean or Alfalfa Sprouts in A-Sprouter }}$}

1. Measure correct amount of seeds to be grown. For both mung bean and alfalfa, 3 tablespoons of seeds were used. Weigh each sample of seeds and record weight.

2. Soak the seeds in a solution of Zero-Tol ${ }^{\mathrm{TM}}$, diluted $50 \mathrm{X}$, for 15 minutes. Then rinse the seeds thoroughly and soak them in DD water for 24 hours.

3. The following day, rinse the seeds several times with DD water and spread the seeds evenly over the sprouter tray.

4. Fill the sprouter to the designated line with the appropriate concentration of $\mathrm{Ag}^{+}$ solution (prepared from $\mathrm{AgNO}_{3}$ ). Each A-sprouter requires about 3 liters of solution.

5. Assemble the sprouter according to the instruction manual and grow seeds under $24 \mathrm{hr}$ light conditions at room temperature.

6. Sprouts are ready to be harvested at the end of a growth period of 7 days. At this time, wash sprouts with tap water 3 times and with DD water 3 times to remove any residue from the outside of the plants.

7. Dry biomass in a Fisher Isotemp ${ }^{\mathrm{TM}}$ Oven (100 series model) at approximately $80^{\circ} \mathrm{C}$ to ensure all water is out of sprouts (the time required is about 15 hours depending on how much biomass is placed inside the oven). Cool, weigh again, and record weight.

8. Obtain a small random portion (approx. $3.5 \mathrm{~g}$ ) of the dry biomass and weigh accurately. 
9. Grind this sample with a mortar and pestle to produce a fine powder.

10. Place this powder in a clean, dry crucible and burn at $700^{\circ} \mathrm{C}$ in a muffle furnace (FB14415M, Barnstead/Thermolyne Corp.) for about 2.5 hrs.

11. After burning, allow ash to cool. Then weigh again and record weight.

12. Store ash in clean, dry containers and place samples in a dessicator until needed.

13. Analyze ash for silver content using an Atomic Absorption Spectrophotometer. (See Appendix I)

\section{$\underline{\text { G.2 Procedure for Growing Mung Bean or Alfalfa Sprouts in S-Sprouter }}$}

1. Measure correct amount of seeds to be grown. For mung bean 2 tablespoons seeds were used, and only 1 tablespoon was required for alfalfa. Weigh each sample of seeds and record weight.

2. Soak the seeds in a solution of Zero-Tol ${ }^{\mathrm{TM}}$, diluted $50 \mathrm{X}$, for 15 minutes. Then rinse the seeds thoroughly and soak them in DD water for approximately 24 hours.

3. The following day, rinse the seeds several times with DD water and spread the seeds evenly over the sprouter tray.

4. Fill the sprouter plate with the appropriate concentration of $\mathrm{Ag}^{+}$solution (prepared from $\mathrm{AgNO}_{3}$ ) until the liquid level just reaches the tray holding the seeds. Each A-sprouter requires about 0.5 liters of solution.

5. Replace lid on sprouter, leaving vents closed and grow seeds under $24 \mathrm{hr}$ light conditions at room temperature. 
6. When seeds germinate (after about three days), open vents on sprouter lid. Add DD water when necessary to maintain a proper water level.

7. Follow procedures as indicated on steps 6-13 of G.1. 


\section{Appendix H. Procedures for Induction and Maintenance of Hairy Roots}

\section{$\underline{\text { H.1 Procedure for Bacteria Culture Initiation and Subculture }}$}

1. Slowly thaw cryogenically preserved colonies of Agrobacterium rhizogenes (ATCC 15834) to room temperature. First, transfer frozen cultures from the freezer to a refrigerator. After the cultures thaw, remove from the refrigerator and allow the bacteria to warm to room temperature.

2. Under aseptic conditions, inoculate Erlenmeyer flasks containing YMB (Appendix B) with several loopfuls of bacteria. If using $250 \mathrm{ml}$ flasks, initial liquid volume should be about $150 \mathrm{ml}$. For $500 \mathrm{ml}$ flasks, use approximately 200 $\mathrm{ml}$ fresh medium.

3. Incubate flasks in the dark at $25^{\circ} \mathrm{C}$, and agitate on an orbital shaker at $100-120$ rpm.

4. Subculture to fresh media every 7-10 days. Pour a small portion (approximately $25 \mathrm{ml}$ ) of the cloudy broth into flasks containing $125 \mathrm{ml}$ (for $250 \mathrm{ml}$ flasks) or 175 $\mathrm{ml}$ (for $500 \mathrm{ml}$ flasks) of fresh YMB. After three successive subcultures, the bacteria are ready for use.

5. Harvest the inoculum within 1-3 days following subculture, when the liquid becomes cloudy with bacteria.

\section{H.2 Establishment of Stevia Plantlets for Hairy Roots}

1. Sterilize Stevia seeds according to the procedure from Appendix A and place them in large baby jars or tall petri dishes on solid 1/2 MS medium (Appendix B). 
2. Initially, use darkened conditions to mimic natural germination of seeds buried in soil. Use a box or other dark container to achieve sufficient darkness. Adjust the temperature to around $25^{\circ} \mathrm{C}$ to initiate growth.

3. After seedlings emerge, transfer jars to an incubator which has 18 hour light $/ 6$ hour dark cycles and keeps plants around $25^{\circ} \mathrm{C}$. Stems are adequately strong and thick after 1-2 months of growth, and plants are ready for infection with bacteria.

\section{$\underline{\text { H.3 Induction and Maintenance of Stevia Hairy Roots }}$}

1. Harvest Agrobacterium rhizogenes 1-3 days following subculture. Place a small amount of this culture in a sterile microcentrifuge tube and centrifuge for several minutes until a pellet of bacteria can be observed at the bottom of the tube.

2. Using a sterile hypodermic needle, inject portions of this bacteria pellet into various sites on the stem of the plantlet. Place plant cultures back into the incubator with 18 hours light $/ 6$ hours dark and a temperature of $25^{\circ} \mathrm{C}$.

3. After 1 or 2 weeks, hairy roots are visible at each infection site.

4. About 3 weeks after infecting plantlets, hairy root growth is sufficient for subculture. Remove segments of stem containing hairy roots using scissors or a sharp scalpel, and place them on fresh $1 / 2$ MS medium.

5. Leave some hairy roots exposed to light, and place others in darkened conditions.

6. Approximately 4-5 weeks later, excise remaining stem segments, and subculture hairy roots onto $1 / 2$ MS medium containing ampicillin to eliminate bacterial contamination. Now hairy roots can be subcultured every 3-4 weeks. 
7. Continue subculturing to MS medium with ampicillin for three passages, and then carry out successive subcultures without the use of antibiotics. For subculturing hairy roots, it is necessary to transfer only a few segments of the hairy root to fresh medium, since growth is rapid.

\section{H.4 Establishment of Periwinkle Plantlets for Hairy Roots}

1. Sterilize periwinkle seeds according to procedures in Appendix A and place them in large baby jars or tall petri dishes on solid Gamborg's B5 medium (Appendix B).

2. Initially, use darkened conditions to mimic natural germination of seeds buried in soil. A box or other dark container is sufficient to achieve darkness. Adjust temperature to around $25^{\circ} \mathrm{C}$ to initiate growth.

3. When seedlings emerge after 5-7 days, transfer cultures to an incubator which has 18 hour light $/ 6$ hour dark cycles and keeps plants around $25^{\circ} \mathrm{C}$. After $1-2$ months of growth, plants are ready for infection with bacteria.

\section{H.5 Induction and Maintenance of Periwinkle Hairy Roots}

1. Harvest Agrobacterium rhizogenes 1-3 days after subculturing. Place a small amount of this cloudy broth in a sterile microcentrifuge tube and centrifuge for several minutes until a pellet of bacteria can be observed at the bottom of the tube.

2. Using a sterile sewing needle, scratch the stem of the plantlet. Use a sterile pipet to inject portions of the bacteria pellet into the wound sites on the stem of the plantlet. Keep plantlets in dark for 12 hours to prevent the bacteria from drying 
out and becoming ineffective. Then continue incubation with the same light cycles and temperature required for growth of plantlets.

3. After 1 or 2 weeks, hairy roots emerge at the infection sites. After hairy roots have grown at least $2 \mathrm{~cm}$, remove segments of the hairy roots using scissors or a sharp scalpel, and place them on fresh $1 / 2$ B5 medium containing ampicillin (Appendix B).

4. Continue subculturing to $1 / 2 \mathrm{~B} 5$ medium with ampicillin for the next three passages, and carry out following subcultures onto medium without ampicillin. 


\section{Appendix I. Preparing Samples for Silver Analysis by Atomic Absorption}

1. Obtain a sample of ash from cells or sprouts weighing approximately $0.025 \mathrm{~g}$. Accurately record the weight. Before collecting the sample, shake the container gently to ensure that the ash is well mixed.

2. Place samples into small Erlenmeyer flasks.

3. To each sample, add a small volume (about $25 \mathrm{ml}$ ) of concentrated $\mathrm{HNO}_{3}$ to dissolve the biomass. Any silver present will also be dissolved.

4. Under a hood, heat samples on a hotplate. Let them boil gently for about 45 minutes without drying up, and then remove from heat.

5. If any insoluble particles remain, filter them out of the solution.

6. Dilute each sample to $100 \mathrm{ml}$ with DI water.

7. Because AA is only accurate for a particular range of concentrations, sometimes additional dilution is necessary. Trial and error has led to a rough guideline for further dilution of samples:

\begin{tabular}{|l|l|}
\hline Types of Samples & Further Times Dilution $(\mathrm{X})$ \\
\hline Sprouts from A-sprouter and suspension & {$[$ (initial ppm)/2]X } \\
\hline Sprouts from S-sprouter & \\
\hline
\end{tabular}




\section{Appendix J. Reactions of Geranium Extract with Silver and Gold Solution}

\section{$\underline{\text { J.1 Geranium Reaction with } \mathrm{AgNO}_{3}} \underline{\text { and }} \mathrm{KAuCl}_{4}{ }^{\mathrm{i}}$}

1. Obtain $9.0 \mathrm{~g}$ of freshly cut leaves. Choose only the most healthy-looking leaves. Remove all portions of stem and leaf stalk, as these are difficult to grind.

2. Using a sharp knife and cutting board, chop the leaves into small pieces. Squeeze the biomass through the $2.0 \mathrm{~mm}$ screen of the TissuePress ${ }^{\mathrm{TM}}$ to further reduce the size of the leaf pieces.

3. Add $45 \mathrm{ml}$ of DD $\mathrm{H}_{2} \mathrm{O}$ to the 9 grams of ground leaves. Rinse the leaf residue from the cutting utensils using small portions of this water. Combine the water and biomass in a $250 \mathrm{ml}$ Erlenmeyer flask

4. Boil the mixture for 1 minute to further disrupt the cells.

5. Decant $10 \mathrm{ml}$ aliquots of the extract into $120 \mathrm{ml}$ glass jars, and add $100 \mathrm{ml} 0.001$ $\mathrm{M} \mathrm{AgNO}_{3}$ or $\mathrm{KAuCl}_{4}$ to each sample. Only four samples could be prepared through this procedure due to loss of water from boiling.

6. Place jars containing reactants under varying environmental conditions to allow for observation of the effects of light and oxygen on the reaction. In each run, one sample was exposed to fluorescent lighting, as well as air. Another was also under light, but had an airtight lid to prevent any oxidation of the solution. The two remaining samples were in darkened conditions; one open to air, and the other tightly sealed.

\footnotetext{
${ }^{\mathrm{i}}$ Steps 3-5 were modeled after procedures given by Shankar et al. (2003a, b).
} 


\section{$\underline{\text { J.2 Geranium Reaction with } \mathrm{KAuCl}_{4}} \underline{\text { Modified to Observe Effects of Boiling Time }}$}

1. Obtain $12.0 \mathrm{~g}$ of freshly cut leaves. Choose only the most healthy-looking leaves. Remove all portions of stem and leaf stalk, as these are difficult to grind.

2. Separate these leaves into 6 equal $2.0 \mathrm{~g}$ samples.

3. Using a sharp knife and cutting board, chop the leaves from one sample into small pieces. Squeeze the biomass through the $2.0 \mathrm{~mm}$ screen of the TissuePress ${ }^{\mathrm{TM}}$ to further reduce the size of the leaf pieces.

4. Add $100 \mathrm{ml}$ of $\mathrm{DD} \mathrm{H}_{2} \mathrm{O}$ to the 2 grams ground leaf. Rinse the leaf residue from the cutting utensils using small portions of this water. Combine the water and biomass in a $250 \mathrm{ml}$ Erlenmeyer flask.

5. Repeat this process for each sample of leaves.

6. Once all leaves have been macerated, boil the mixtures for different time periods. For this experiment, boiling times of 1, 5, 10, 15, and 20 minutes were investigated. For one sample, the procedure was carried out without heating or boiling.

7. After boiling, remove immediately from heat and let solutions cool to room temperature.

8. Filter solutions under vacuum filtration to remove leaf matter.

9. To correct samples for evaporation due to heating, dilute each sample to $100 \mathrm{ml}$ in a volumetric flask.

10. Transfer $10 \mathrm{ml}$ aliquots of the samples to $120 \mathrm{ml}$ glass jars, and add $100 \mathrm{ml}$ $0.0001 \mathrm{M} \mathrm{KAuCl}_{4}$ to each sample.

11. Place jars in full fluorescent lighting, with lids tightly sealed. 
$\underline{\text { J.3 Geranium Reaction with } \mathrm{KAuCl}_{4} \text { Modified to Observe Effects of Boiling After }}$ $\underline{\text { Leaves are Filtered Out }}$

1. Follow procedures 1-3 from J.2.

2. Add $100 \mathrm{ml}$ of $\mathrm{DD} \mathrm{H}_{2} \mathrm{O}$ to the 2 grams ground leaf. Rinse the leaf residue from the cutting utensils using small portions of this water. Combine the water and biomass in a beaker.

3. Filter to remove leaves from the solution.

4. Repeat this process for each sample of leaves.

5. After all extracts have been prepared and leaves have been removed, boil the mixtures for different time periods. For this experiment, boiling times of $1,5,10$, 15, and 20 minutes were investigated. For one sample, carry out the procedure without heating or boiling.

6. Carry out procedures 7 and 9-11 from J.2. 


\section{Addendum}

The spectra generated by the Spectronic Genesys 5 Spectrophotometer revealed much information relevant to the reaction of $\mathrm{AgNO}_{3}$ and $\mathrm{KAuCl}_{4}$ with the three cultivars of Pelargonium graveolens. Although the spectra data have been stored in the spectrophotometer, technical difficulties have prevented many figures from being included in this document. At this point, only some spectra are accessible, and the following figures were selected from the available spectra in attempt to convey as much significant information as possible.

One characteristic of the spectra that were produced that should be known with regard to the analyses of the spectra is the relationship between nanoparticle concentration and the absorbance. It was observed that when the diameter of gold nanoparticles is constant, the absorbance is directly proportional to the concentrations of gold in the solution. Figure A.1 displays two spectra that were produced using samples of the same gold nanoparticle solution, but (a) corresponds to a sample that was analyzed without dilution and (b) shows the spectrum of a sample that had been diluted $2 \mathrm{X}$. This figure shows that the plasmon resonance bands for the samples are very similar, and the absorbance maxima occur at nearly the same wavelengths, but the intensity of the peaks are much different. For the sample that has been diluted $2 \mathrm{X}$, the maximum absorbance is 0.182 and the sample that has not been diluted shows a maximum absorbance of 0.361 , almost exactly double. 
(a)

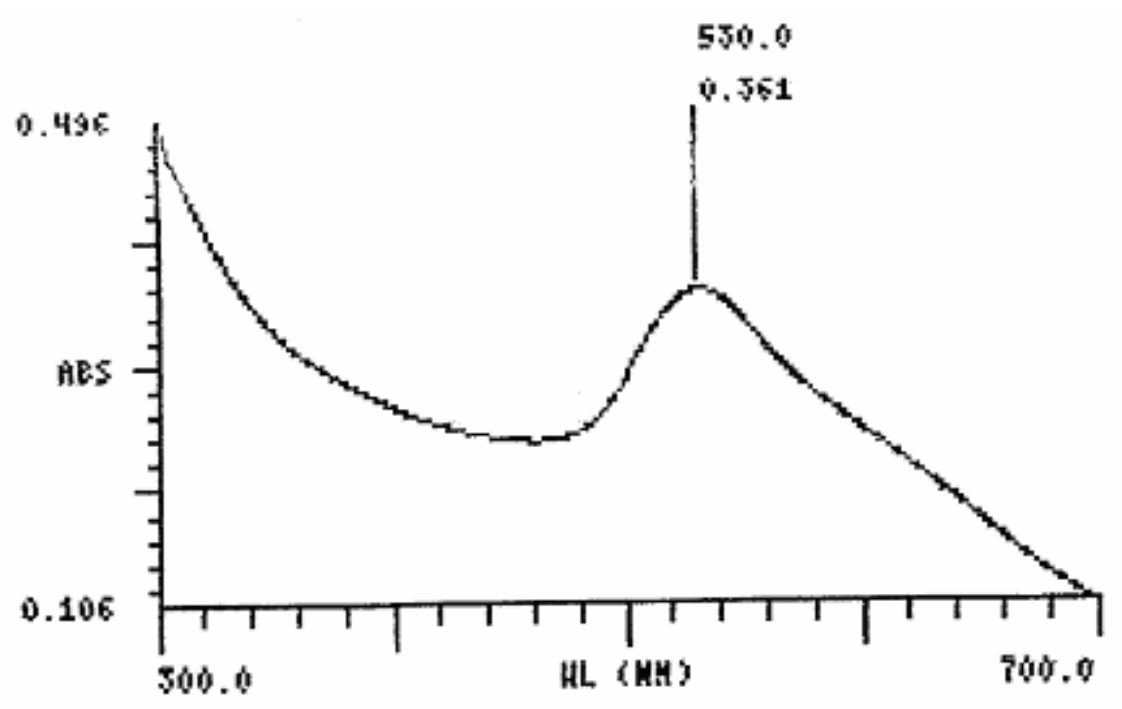

(b)



Figure A.1. Absorbance spectra of samples of a solution containing gold nanoparticles after reaction of RR extract with $\mathrm{KAuCl}_{4}$, (a) shown without dilution, and (b) diluted $2 \mathrm{X}$ relative to the sample represented in (a). 